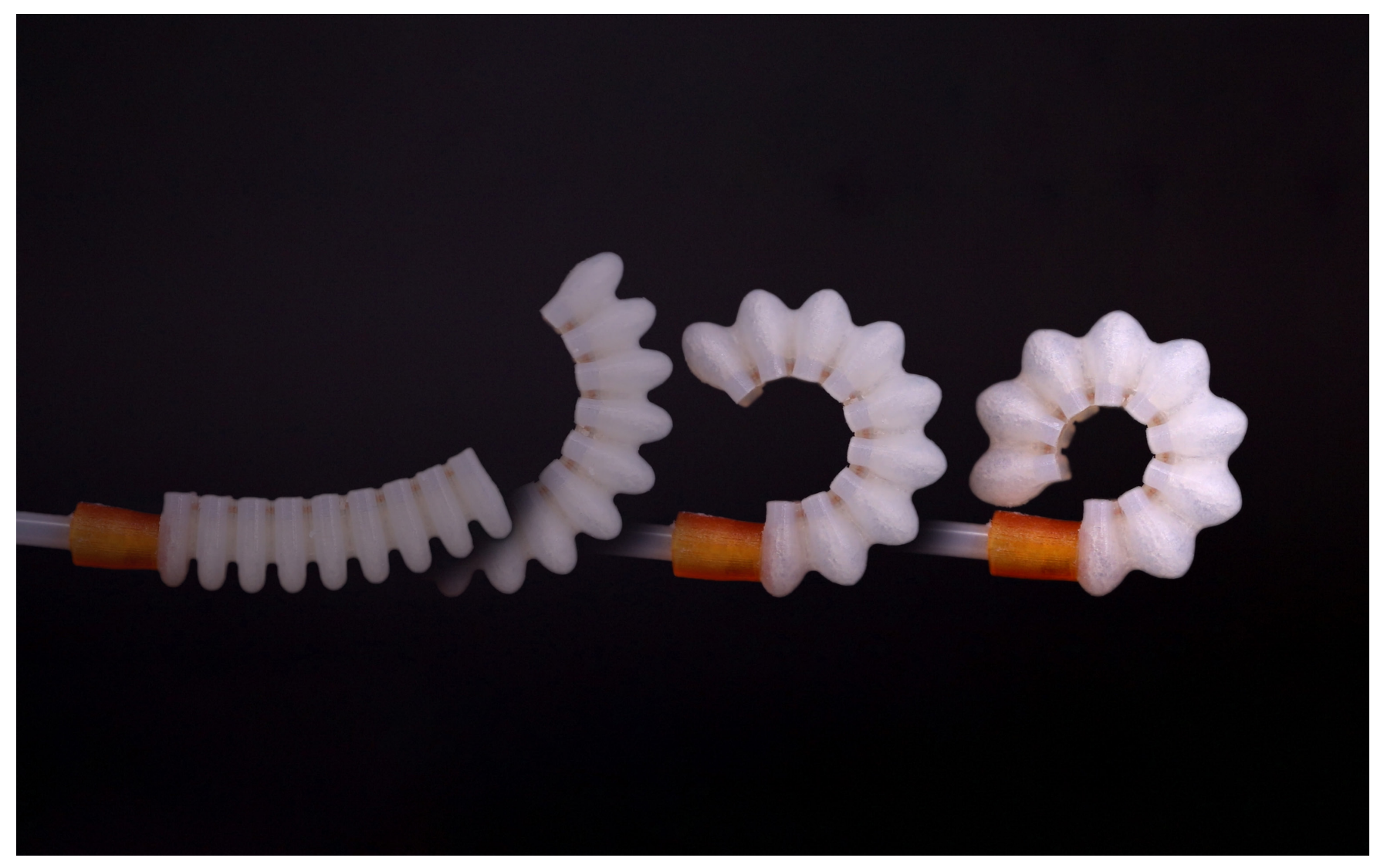

Well, Actuate(ly)...

Parametric Multi-Material 3D Printed Soft Robotics 
A research portfolio submitted in partial fulfilment of the requirements for the degree of Master of Design Innovation.

\section{Patrick Coulson}

Supervised by Tim Miller

Victoria University of Wellington

School of Design

2020 


\section{Abstract}

In recent years, soft robotics has gained wide interest in the research field and has also garnered some commercial success. This is because soft robots are comprised of soft materials that have inherent compliance which lends them to a wide variety of applications that are not suited to traditional hard-bodied robots.

Soft robots are generally created using a casting process, which comes with limitations to the geometry due to the removal of the cast body from the mould. This research seeks to enhance the capabilities of soft robotic limbs using multi-material Polyjet printing - a recently developed additive manufacturing technology - which allows for geometric freedom and variable materials within a singular soft 3D print which is not feasible using other fabrication methods.

This research draws inspiration from natural mechanisms such as muscular hydrostats, to enable the exploration of singular channel soft robots that exhibit bending, twisting, elongation, and expansion all in one 3D print. The geometric freedom and variable materiality of the Stratasys J750 produce actuation results for each motion that cannot be easily replicated using traditional fabrication techniques. The printable materials of the Stratasys $\mathrm{J} 750$ were found to have tendencies to tear upon inflation, however, a large array of prints with complex geometry were able to successfully actuate despite this. In some areas, results outperformed actuators made using other fabrication techniques, as was particularly evident in the twisting actuators. Through fine-tuned parametric control with equation driven modelling, this portfolio presents a method for sof robotic design and construction that can produce a limb with multiple motions and up to 5 axes of movement that can be tailored to specific pre-defined applications. 


\section{Acknowledgements}

Thanks to the National Science Challenge and the MADE research stream for supporting this project.

Thank you to my supervisor Tim Miller for the continuous support guidance, and commitment to this project.

A big thanks to my family for their everlasting belief in my work and in particular Alan, and for his willingness to help at a moment's notice.

To Bridy for always giving me the help, support, and encouragement I needed and for always keeping my spirits high.

To the MDI cohort for creating a positive work environment that always stuck together and motivated one another. A big thanks to the VUW staff,

in particular Phil, for the technical guidance and assistance. 


\section{Table of Contents}

\begin{tabular}{|c|c|}
\hline Methodology & 5 \\
\hline Literature review & 11 \\
\hline Introduction & .13 \\
\hline History & 14 \\
\hline Soft robotics and 3D printing & 16 \\
\hline Sequencing of motion in soft robotics & 18 \\
\hline Synthesis of the literature & 19 \\
\hline Biomimicry & 20 \\
\hline Experimentation & 23 \\
\hline Introduction & 25 \\
\hline Adhesion testing.................... & 27 \\
\hline Biological inspiration & 33 \\
\hline Software & 35 \\
\hline Balloon testing ......... & 39 \\
\hline Soft robot print sets. & 41 \\
\hline
\end{tabular}

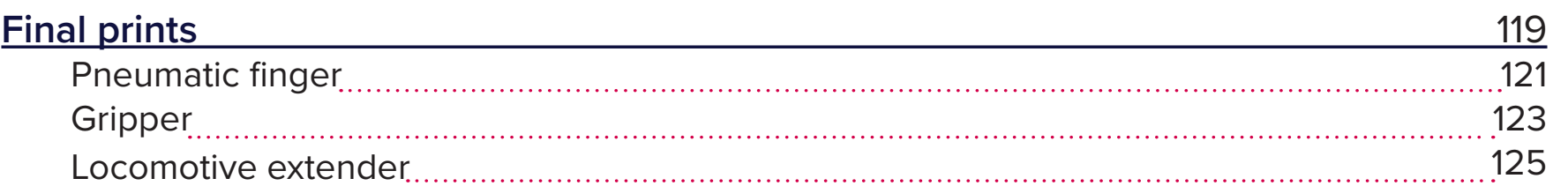

\begin{tabular}{ll} 
Discussion & 127 \\
\hline Summary & 129
\end{tabular}

\begin{tabular}{ll} 
Summary & 129 \\
\hline & 131
\end{tabular}

Limitations

Future research 133

Implications

Applications $\ldots 136$

\begin{tabular}{ll} 
Conclusion & 137 \\
\hline
\end{tabular}

Figure list and references 
I N TRODUCTION 
Soft robotics is a research field which has expanded rapidly over the last two decades, which utilises soft materials to manipulate irregularly shaped objects. Where conventional robotics are excellent for interacting with regular environments and defined geometries, soft robots thrive in the ability to adapt to their environment through their geometry and actuation method without reliance on digital sensors (Rus \& Tolley, 2015). This gives them a wide range of possible applications that rely on compliance with irregular objects and environments. Many researchers have shown the dexterity of soft robots for gripping various organic objects, assistive stroke rehabilitation, and locomotion of unstructured terrain (Rus \& Tolley, 2015). To further the wide range of potential applications for soft robots, research groups focus on optimising the motion of the actuators, and to increase the catalogue of possible motions.

Typically these robots are moulded and cast using silicone rubber. This research investigates the possibilities of using multimaterial Polyjet 3D printing as an alternative fabrication method for soft robotic systems.
3D printing in soft robots has recently proven to introduce fine control over the motion of the actuator (Bartlett et al., 2015; Peele et al. to replicate with a casting process. This fabrication technique is very new, and because of this, the current research only covers small amount of what could be possible (Gu et al., 2018, p.258). Multi-material 3D printing offers new possibilities for programmable articulation in soft robots as it gives greater control over geometry and materiality than other fabrication processes.

This research encompasses a wide range of experiments that are all pneumatically actuated through one channel, all capable of vastly different motion. Through a number of iterations, this research demonstrates nove actuators capable of high-performance motion which have not been widely researched across the field of soft robots.

At a crucial stage in the research, this project was significantly impacted by Covid-19. This is mentioned in further detail in the limitations section of the discussion chapter.

\section{Portfolio overview}

\section{Methodology}

This chapter details the aims and objectives of this portfolio and the strategies used to achieve them.

\section{Literature Review}

This chapter gives context to the portfolio and assesses the gap in the literature that this research aims to fill. A history of soft robots, soft robots and 3D printing, and sequencing of motion in soft robots are all reviewed.

\section{Biomimicry}

Biomimicry has been used to ideate in the design stage of this portfolio. This chapter covers the relevance of adhesion and muscular structure in this portfolio.

\section{Experimentation}

The experimentation phase shows a wide range of Polyjet printed soft robots and how software and biomimicry have been used to inform the design.

\section{Final Prints}

This showcases the set of demonstrators with the most success as an output of the experimentation phase of the research.

\section{Discussion}

This chapter evaluates the success of using multi-material printing for soft robots, as well as discussing the limitations and possibilities for future research. 
METHODOLOGY 


\section{Research Question}

How can multi-material 3D printing be used to fabricate singular channel pneumatically actuated soft robots capable of complex actuation for advanced applications?

\section{Aims \& Objectives}

1. To investigate the nature and complexity of actuation currently achievable in soft robotic systems.

a. Analyse and critically assess the actuation achievable by soft robotic systems across the literature.

b. Explore and identify biological precedents that present knowledge in variable materiality and geometry of movement, as well as any other areas that are determined as having the potential to improve the performance of soft robots.

2. To establish and develop techniques for multi-material 3D printing as a method of fabrication for soft robots.

a. Explore and evolve a range of soft robotic concepts.

b. Conduct a series of experiments exploring the opportunities and limitations of multi-material 3D printed soft robots.

c. Evaluate and develop multi-material 3D printed soft robots to achieve complex actuation for determined applications.
This portfolio is structured and written with a research through design approach as defined by Frayling $(1994$, p. 5). Design research can be separated into three categories; research about design, research through design, and research for design (Frankel \& Racine, 2010; Frayling, 1994; Hanington \& Martin, 2012; Milton \& Rodgers, 2013). Research through design follows a similar process to a conventional design process, however, research through design can be distinguished primarily through documentation; design experimentation along with reflection and contextualisation within the field are all written about in thorough detail (Milton \& Rodgers, 2013) where typically the only evidence of design through process is in a final object produced (Frayling, 1994). This portfolio largely follows an iterative process of the creation of physical prototypes utilising a research through design approach. As described by Hanington \& Martin (2012), research through design encompasses process which begins with secondary research to contextualise the study as outlined by Aim 1. The past and present landscapes of soft

robotic research have been assessed to gain an understanding of universal concepts and principles in soft robotics that can be applied to this research. Literature and precedent review are key research methods for determining the possible areas of influence or potential for the advancement of soft robotics through the utilisation of the geometric freedom and variable materiality through multi-materia printing on the Stratasys J750. This background research is essential in focussing the scope of the project and obtaining knowledge which is then drawn upon during the primary research phase of the portfolio. Biological mechanisms have also been evaluated to gather knowledge about movement across natural structures, with the hope that this knowledge can be transferred across to inform geometry and variable materiality for soft robotic concepts. The second stage of this research, outlined by Aim 2, moves into the primary research of this portfolio. Primary research has been carried out to develop soft robotic concepts capable of a range of movements. This has been achieved through the following workflow:

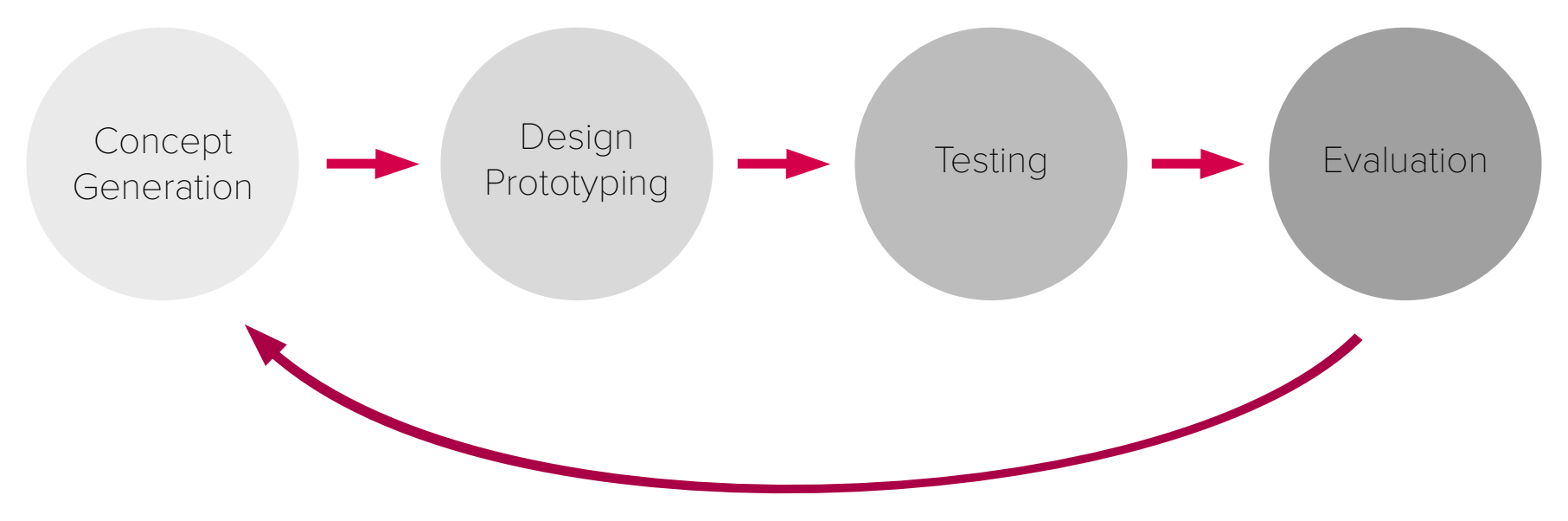


This workflow has been created to explore the possibilities of soft robots that utilise the geometric freedom and variable materiality of printing on the Stratasys J750 in an efficient and deeply explorative manner as it prioritises a large number of iterations to be produced. Through the repetition of this workflow, concepts can easily be developed through the experimentation and evaluative research phases as these methods generate both comprehensive qualitative and quantitative data. Concept Generation is carried out through sketching and 3D modelling to produce soft robotic concepts that explore a variety of movements. Parametric modelling methods are employed to streamline an iterative design process where changes in geometry and materiality can easily be refined through fine-tuned parameter control. By employing these methods, improved control over geometry and materiality is obtained, and the iterative process is accelerated to produce a high quantity of iterations. Design
Prototyping is carried out through fabrication using the Stratasys J750 3D printer to materialise the concepts into physical forms. A small amount of post-processing of the prints is required before the experimentation phase.

Experimentation is used to gather quantitative cata from the prototypes to obtain information abouttheirsuccessin achieving their respective motions. The experimentation covers the study of pressure, geometry, and variable materiality as functions of the soft robots' actuation. Evaluative Research (Hanington \& Martin, 2012) is used to determine the points of success and failure in the prototypes, and what can be changed in future iterations. A se of soft robots capable of different motions that effectively exhibit the advantages of using the Stratasys $\mathrm{J} 750$ as a fabrication method are then combined to complete objective $2 c$. This final complex soft robot is a demonstrator for the precision, optimisation, and range of motions that can be printed using multi-material Polyjet 3D printing. 


\section{Introduction}

When we think 'robot' we probably think of an autonomous steel machine with intricate componentry that operates using sensors or predefined actions through motors - a machine that offers movement at higher speeds and higher accuracies than in their biological counterparts. This idea has been challenged in recent years as a field of robotics labelled 'soft robotics' has surfaced. The field encompasses the use of soft or extensible bodies with inherent compliance (Trivedi et al., 2008) which are used to traverse or manipulate their surrounding environment (Rus \& Tolley, 2015). Unlike hard-bodied robots, soft robots move in a way that seems organic, which is achieved through their adaptation and compliance. Soft robots offer certain advantages over conventional hard-bodied robots, and in turn are better suited to specific applications (Trivedi et al., 2008).

\section{History}

Soft robotic applications can be split into three main categories: locomotion, biomedicine, and gripping.

Locomotion has been studied in many different scenarios, frc to swimming. A paper published in PNAS demonstrating a multi-gait soft robot capable of crawling through a small gap (Shepherd et al., 2011) is one of the most widely known soft robotics papers. The soft robot was cast using a silicone elastomer and it contained five separate actuation channels. The authors were able to achieve locomotion of their robot by sequencing the actuation of each channel to create a gait similar to crawling. They proved the dexterity of the finely controlled soft robot by optimising its gait to navigate under a glass sheet. George Whitesides, a prominent figure in the soft robotic field, believes that this technology could be utilised to navigate through hazardous or inhabitable terrain such as nuclear disaster sites (Whitesides, 2016) as a significantly cheaper and easier to manufacture alternative to conventional hardbodied reconnaissance robots. The design of the robot shown by Shepherd et al. (2011) has since been expanded upon by other researchers. A notable example is Tolley et al. (2014) who were able to scale up the design and bring the pneumatic system on board so that it would be completely untethered. This paper is significant as it shows that scaling a pneumatic actuator up will affect either the material density of the robot or the pneumatic input pressure in order to achieve the same level of movement as its smaller counterpart. This paper also details the moulding and casting process used to fabricate their robot. Multiple materials and fabrication processes were required for the creation of the mould.
The mould was assembled from multiple sheets of both laser cut and water jet cut materials, the rubber composite used for the main body was degassed, and it was then poured into the mould. In contrast to the locomotion achieved by both Shepherd et al. (2011) and Tolley et al. (2014), Marchese Onal, \& Rus (2014) demonstrated a soft robotic fish capable of swimming and performing rapid escape manoeuvres. This robot included an onboard gas $\mathrm{CO}_{2}$ gas cylinder for actuation, meaning that, like the soft robot created by Tolley et al. (2014), the soft robot could move unconstrained by any tether. The robo actuated through internal inflation chambers on either side of the fish, which allowed it to bend in Although the swimming gait was determined to be suboptimal (p.82), this research demonstrates the possibilities of soft robotic locomotion in a vastly different environment to what had been previously researched.

Many research groups have been studying the possibility of using soft robots for biomedicine. This is a field of significant interest for soft robots because their compliance allows for interaction with human tissue without damage (Cianchett et al., 2018). Although soft robotic systems have been developed for the assistance of a range of body parts, hand rehabilitation has attracted the most attention (Cianchetti et al. 2018, p. 147) Yap, Jeong Hoon Lim, Goh, \& Yeow (2015) show a rehabilitation glove comprised of cast silicone elastomer soft robotic actuators with localised bending. The bending is located over the knuckles of the fingers which, when pressurised, assist in the bending of the fingers. The localisation of the bending was achieved by having bellows in the chamber geometry only in the regions 
were bending was required. With this, Yap et al. (2015) were able to generate a force output of $3.59 \mathrm{~N}$ for gripping. Polygerinos, Wang, Galloway, Wood, \& Walsh (2015) were able to achieve an output force of approximately $8 \mathrm{~N}$ for a similar rehabilitative glove, however, the method for localising the bending around the knuckles was less precise than that demonstrated by Yap et al. (2015). Outside of hand rehabilitation, Ranzani et al. (2015) were able to create a bioinspired manipulator for minimally invasive surgery which was able to manoeuvre through the use of segments along the robot which each generated its own motion. This was done in order to navigate through the human abdomen with precision. A braided sheath with bellows geometry was fitted around the system in order to constrain it from excessive radial expansion (Cianchetti et al., 2013). This paper also identified that scaling soft robots is a big issue for biomedical applications as it often requires a radical change in the fabrication process as well as redimensioning of the geometry.

The most documented and widely researched application for soft robots revolves around gripping. Soft robotic grippers are used to pick up irregularly shaped or organic objects by having actuators conform around the surface of the object. Early examples show simple bending actuation of grippers to pick up delicate objects such as eggs demonstrated by llievski et al. (2011). This research helped to pave the way for future soft robotic research as it comprehensively demonstrated how the distribution of material and changing geometry altered the performance of the actuators and implemented these findings into a versatile soft robotic gripper with a textured surface which increased the grip performance. This research quickly developed into soft robots capable of bending in 3 dimensions rather by increasing the number of pneumatic inputs and creating separate controllable regions of inflation to grasp objects with delicate features (Martinez et al, 2013; Ranzani et al. 2015). Martinez (2013) also used this technique to demonstrate how 3-dimensional movement could be used for biomedical applications with fine-tuned precise navigation. As demonstrated by Galloway et al. (2016), gripping of delicate features can extend to deep-sea reef sampling, as shown by their soft gripper. To be able to use hard-bodied robots for deepsea sampling, sophisticated force feedback is required to minimize damage when grabbing (Galloway et al., 2016) - so the compliance of soft robots is an ideal replacement. In order to minimise damage, tests were done to assess the pressure distribution of the soft robo across the surface that they were gripping. a layer of memory foam to the gripping surface of the soft robot created a more even pressure distribution across the surface (p. 30), which would, in turn, lower the risk of damaging a reef sample.

Soft Robotics Inc has commercialised soft robotic grippers for packaging application (MGrip, n.d.). These soft robots work as end effectors for a hard robot. The hard-bodied robot is used to position the robo object is trying to grab, and the soft robotic end effectors grab the object and put it down.

\section{Soft robotics and 3D printing}

In the last five years, the use of $3 D$ printing technologies has become increasingly more prominent for the fabrication of soft robots. 3D printing offers advantages over a moulding and casting process in the accuracy and complexity of the output actuation (Drotman et al., 2017, p.5532; Hong Kai Yap et al., 2016, p. $145)$, however, each 3D printing process comes with its own advantages and limitations.

As outlined by $\mathrm{Ngo}$ et al. (2018), there are many different methods for 3D printing. One of the most inexpensive and accessible 3D printing methods, Fusion Deposition Modelling, extrudes a heated layer of filament onto a print bed which is then built up layer by layer on top of itself. This method is good for prototyping, however, it is limited by its accuracy and anisotropic properties ( $\mathrm{Ngo}$ et al., 2018). This method was demonstrated to have capabilities in the soft robotic field by Yap et al., (2016), which used a commercially available filament to fabricate a dual-channel actuator capable of bi-directional bending. The filament used exhibited repeatable actuation; however, high pressure was needed for actuation as the material had a much lower elasticity than elastomers commonly used for soft robotic fabrication with a moulding and casting process.

Paste printing follows a very similar process to Fusion Deposition Modelling but without heating the material before extrusion. Paste printed materials often harden after extrusion for the next print layer to be extruded on top of it to build up a structure. Yirmibesoglu et al. (2018) developed a silicone elastomer paste printer for soft robots to overcome the limitations of commercially available 3D printer materials. They were able to produce soft robots that had a similar performance to cast silicone soft robots. However, the extrusion process has inherent limitations with the complexity of the soft robot, as it is not suited to overhanging geometries.

Stereolithography was the first developed 3D printing technology and it can achieve much higher print resolution than with Fusion Deposition Modelling. Stereolithography works by curing resin from a bath with an UltraViolet laser layer by layer as the object is being drawn out of the bath to create 3-dimensional forms (Ngo et al., 2018). This method was used for soft robotics by Peele et al. (2015) in which detailed air chambers allowed for complex movements. Although Martinez et al. (2013) achieved similar multi-chamber actuation by casting silicone rubber, the tentacle could not manoeuvre at the same level of precision because of the inherent geometrica restrictions on the chamber complexity in the moulding and casting process which can impact the actuation accuracy. The main drawbacks of this printing method include its high cost and long print times (Ngo et al., 2018). This is why Peele et al. (2015) were only able to create small scale soft robots. Peele et al. (2015) also found that the photopolymerisable resin used was prone to tearing which created air leaks.

Digital Light Projector printing is similar to Stereolithography where a resin is cured from a bath when exposed to Ultra-Violet light. However, a digital projector is used to flash an image across the entire layer rather than using a laser that needs to be directed at a specific region of a layer to cure it (SLA vs. DLP, n.d.). This allows for faster build time than Stereolithography and no disruption 
to build times with higher density models. Ge et al. (2018) used Digital Light Projector printing to fabricate a soft robotic gripper at a high resolution compared to other examples of 3D printed soft robots. This allowed for a very small gripper to be fabricated without compromising its functionality. The material used for this gripper has a $170 \%-220 \%$ elongation at break (Ge et al., 2018) which is poor compared to the $900 \%$ elongation at break of the silicone elastomers used with cast soft robots (EcoflexTM 00-30 Product Information, n.d.). Patel et al. (2017) developed a photopolymerisable elastomer for Digital Light Projector printing which exhibits a $1100 \%$ elongation at break, although this is not commercially available.

Inkjet printing works by depositing drops of Ultra-Violet curable resin onto a print bed and curing it once a full layer has been deposited. The drops of resin are deposited from a nozzle into their specific locations unlike Stereolithography and Digital Light Projection which draws material from a bath (Barclift \& Williams, 2012). As a result, some Inkjet printers are capable of depositing multiple materials of varying flexibility and softness into the same print. Bartlett et al. (2015) utilise this technology to create a soft robot capable of jumping using on-board combustion. Complex geometry was able to be created using multiple printed materials to maximise performance. More recently, this technology is starting to be used for air pressurised soft robots. Zhu et al. (2019) illustrated how an Inkjet fabrication process significantly decreases the fabrication time and streamlines the process (p. 509). This is then proven through the creation of a soft robotic gripper using Inkjet printing that is used as an end effector on a robotic arm to grasp objects (Zhu et al., 2019). Soft robots have also been created using this technology exploring embedded Shape Memory Polymers (Zhang et al., 2019) and resistive sensors (Shih et al., 2019). Fabrication of soft robots through Inkjet printing allows for geometric freedom and multi-material capabilities, however, this is yet to be thoroughly explored. The soft robo designs using this technology primarily stil present designs similar to those which were created to suit a moulding and casting process (Shih et al., 2019; Zhang et al., 2019; Zhu et al., 2019). This is no longer necessary as the main advantage of Inkjet printing is that it allows for vast freedom of design and is not at all restricted by the same limitations as a casting process. Schaffner et al. (2018) present multimaterial 3D printed soft robots that exhibit geometry dissimilar to that used in a casting process by using silicone inks with tunable elasticity. The soft robots presented exhibit individual examples of contraction, bending, grabbing, and twisting motions by creating an anisotropic strain achieved through sections of variable material stiffness (p.2).

\section{Sequencing of motion in soft robotics}

Sequencing of motion refers to the configuration of an array of different motions within a single soft body.

The sequencing of motion in soft robotics is very commonly used to create locomotion. Tolley et al. (2014) demonstrated how the sequencing of different segments of a soft robot can be used to achieve different gaits. Both an undulating and an ambulating gait are demonstrated in this paper. This was achieved by the individual control of a pneumatic channel for each segment. This technique was demonstrated as early as Shepherd et al. (2011), however, Tolley et al. (2014) were able to further expand on the variation in gait patterns that were possible through the individual control of each segment of inflation.

Sequencing of motion is less commonly used in soft robotics for grippers. Peele et al. (2015) show a Stereolithography printed soft tentacle with four controllable pneumatic channels that allow for 3-dimensional motion. Peele et al. (2015) utilise 3D printing in order to achieve much finer control over their soft robot than previous examples of 3-dimensional motion in soft robotics.

Sequencing of a range of motions (e.g. twisting, bending, and extension in one soft body) is not widely documented, however, the examples of this are usually directed toward applications in biomedicine. Ranzani et al. (2015) utilise sequencing of bending and elongation as well as stiffening and softening for minimally invasive surgery. Sequencing was utilised for the specified purpose of grabbing an organ, and the individual motions were sequenced in accordance with this. Polygerinos et al. (2015) show bending, twisting, and extension motions for a stroke rehabilitation glove. These motions are sequenced by localising the strain restricting layer which in this case is a fibre, along an otherwise uniform actuator to comply with the different movements that occur when grasping with fingers.

The example of sequencing with the most extensive research and experimentation was carried out by Connolly et al. (2015) where the impact of fibre placement in soft robots was examined to create a soft robot that exhibited localised bending, extension, expansion and twisting to perform a defined task. This robot was used as a proof of concept for the principles determined to create each motion with fibre reinforcement. It exhibited a sequence of extension and expansion to achieve peristaltic locomotion inside a tube and further exhibited bending and twisting to perform its specified task. This research was expanded on to focus on matching the path of actuation to a pre-defined trajectory using mathematical models (Connolly et al., 2017). This research demonstrates the importance of the configuration of the strain restricting regions in soft robotics, and it presents a streamlined method for sequencing different motions. However, it is limited in the performance of the actuators as the change in the type of motion is achieved purely through the placement of the strain restricting regions and not by altering the actuator geometry which could further enhance the performance of each segment. This is particularly evident in the extending and twisting motions. 


\section{Synthesis of the literature}

The primary advantages that soft robots have over hard-bodied robots are:

Compliance: Soft robots have inherent compliance because of the soft materials they are made from, and this lends them to applications where interaction with irregular or organic environments and objects is crucial. Hard-bodied robots would need a number of assembled hard components and carefully considered geometry to pick up a delicate object such as an egg. A pneumatically actuated soft robot can achieve this with simple geometry and a singular pneumatic input (llievski et al., 2011, p.50).

Safety: Conventional robots and humans are typically in separate workspaces in an assembly line because the robots operate at high speeds and forces which can be very dangerous to humans (Krüger et al., 2009). Soft robots typically operate with considerably lower force outputs and are constructed with soft material which lends itself to working alongside humans in a safe environment.

Minimal assembly needed: Soft robots are typically fabricated with a moulding and casting process meaning that they are operational once the material has cured.

The process for creating a soft robot varies greatly from that of a hard robot. Hard-bodied robots consist of a vast number of hard components which are carefully assembled along with sensors, servo motors, and various other electronic components which need to be configured to make the robot move in the desired way. Soft robots rely on their geometry and materiality to determine the way in which they move. Soft robots are conventionally fabricated by pouring a silicone elastomer into a mould. Once the elastomer has set and the robot is taken out of the mould it is fully functional, and it needs only to be connected to an actuation system.

Soft robots are typically actuated in one of two ways; pneumatically or electrically (Trivedi et al., 2008). A pneumatic system works through the expansion or contraction of interna channels which force the robots' geometry to move in a designed way (Figure 2). The electrical alternative is through the use of electroactive polymers which contract when certain voltage is passed through them. Using a pneumatic input is often preferable as these are commonplace (i.e. a bike pump) and are safer as there is no risk of electrocution.

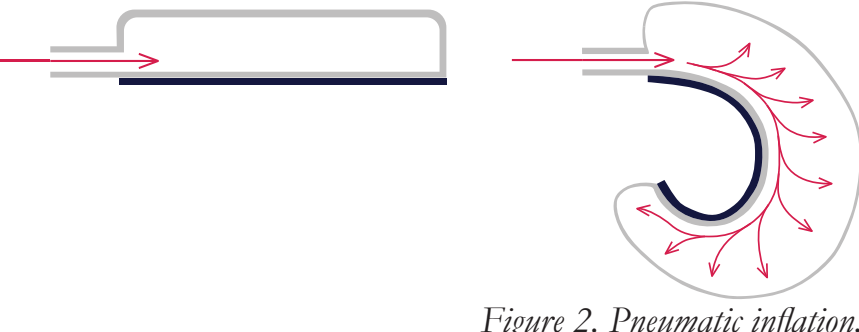

For pneumatically actuated soft robots, the geometry used for actuation can be separated into two categories; bellows-type and what can be described as 'boa-type' (Galloway et al., 2016). A boa-type soft robot consists of a singular air channel and movement is achieved by using a strain restricting layer. The placement of this restricting layer will change the inflated form of the robot. A bellows-type soft robot has air chambers which, when inflated, apply a force on one another, aiding the expansion in a specified direction. These two types of actuators are most documented in bending actuators, as shown in Figure 3 .
Although the type chosen will impact factors such as repeatability of actuation, required input air pressure, and output force of the soft robots' actuation, there are no definitive trends between the two types that can be found across the literature as the geometries are very case-specific.

\section{Biomimicry}

In design, inspiration is commonly taken from certain aspects of our natural world such as forms, behaviours, and mechanisms. The method of incorporating this technique into design research is commonly referred to as 'biomimicry' or 'biomimetics'. According to Benyus (1997), biomimicry can be implemented on three levels; mimicking of natural form, process, and systems. By analysing biological examples, new knowledge can be gained to aid and optimise designs throughout the design process. Schaffner et al. (2018) show a successful example of this in the field of soft robotics at a research level where multimaterial robots were fabricated to mimic the motion created by an elephant's trunk. Festo has also successfully shown the possibilities of soft robotic biomimetic design at a commercial level with their Bionic Handling Assistant (Festo, 2012) and more recently their Bionic Soft Arm (Festo, 2019). Biomimicry, however, has no universal set of guidelines for implementation, therefore it must be used in a carefully considered way. Where design and engineering can be aided by referring to theory, biomimicry is case-specific and each project must employ biomimicry with a fresh

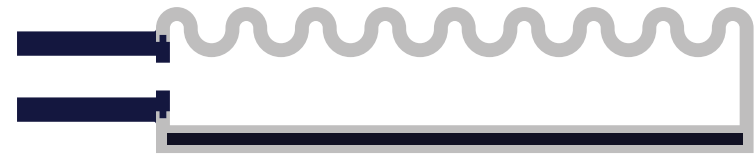

4

Figure 3. Bellows and boa-type actuators.

approach (Vincent et al., 2006).

For the purpose of this portfolio, biomimicry has been studied to analyse how materiality and geometry are utilised. To separate the areas of biology to determine their relevance to this portfolio, they have been categorised in accordance with the physical scale at which they function. At the smallest size, surface adhesion in nature is the way in which animals grip to surfaces. Next is muscular structure, which dictates how the muscles of an organism facilitate movement. Gait looks at the resultant movement that is generated through the organism's muscular structure. At the largest scale, locomotion is defined as how the movement of the organism can allow for travel from place to place.

\section{Adhesion}

For soft robots to interact with their surroundings, good grip strength can significantly increase their performance (Glick et al., 2018) which makes surface adhesion an area of significant interest for soft robotics. Surface adhesion also typically occurs at a 


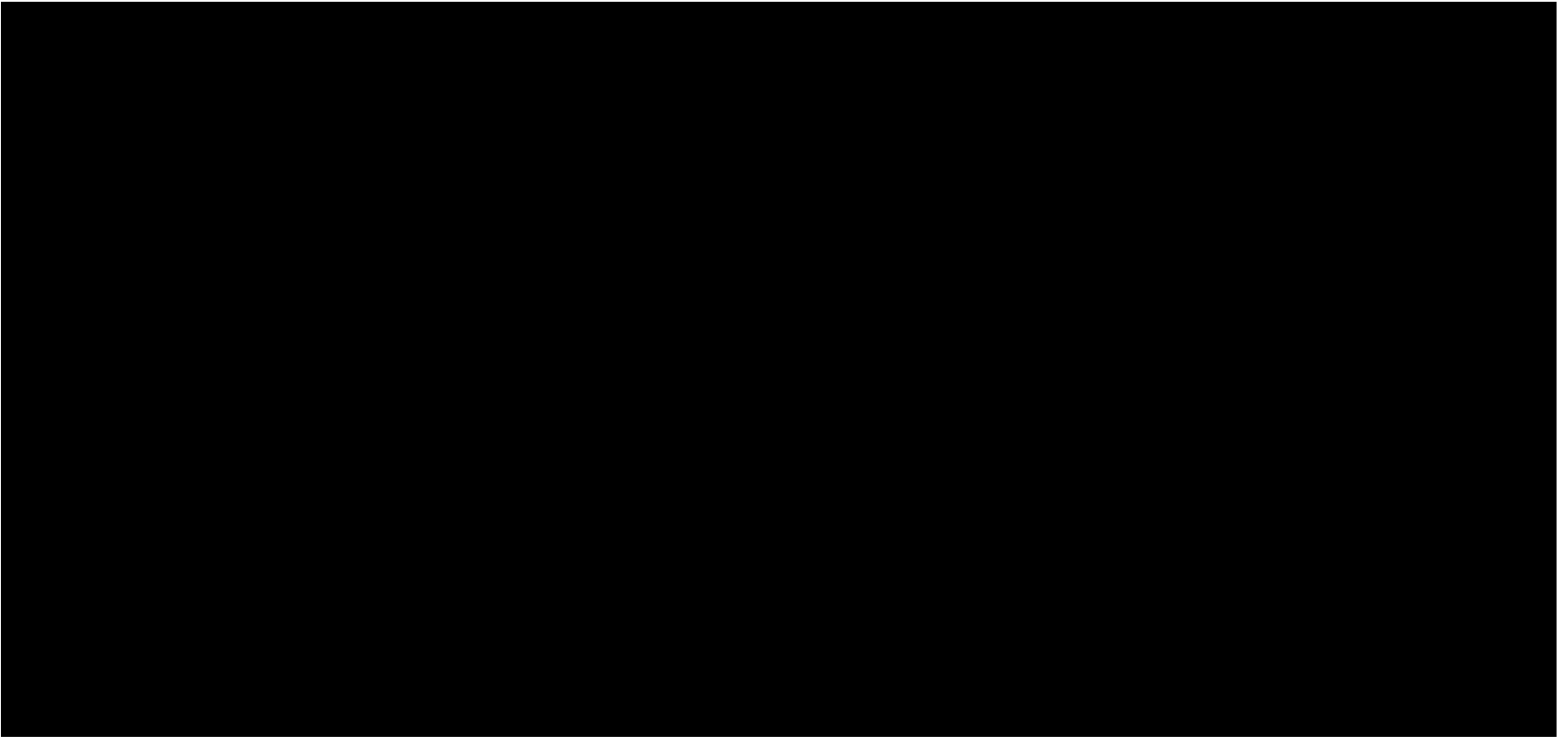

Figure 4. Description of different types and characteristics of main adhesive interactions: Adapted from Heepe \& Gorb (2014).

scale where the resolution of the Stratasys J750 3D printer is capable of printing which makes it of particular interest when exploring the capabilities of this machine for the purpose of this portfolio.

Adhesion in nature is defined as an interaction between two surfaces in which one of the surfaces has a system to create attachment (Arzt et al., 2003; Gu et al., 2016). Adhesive forces can be split into four main categories (Gu et al., 2016); surface and field forces more commonly known as 'dry adhesion' (Autumn et al., 2002; Bhushan, 2009), material bridges or 'wet adhesion' (Federle et al., 2002; Hanna et al., 1991; Vogel et al., 2010), mechanical interlocking, and suction forces.

Dry adhesion often operates using van der Waals forces such as can be found in geckos (Autumn et al., 2002; Rizzo et al., 2006). Geckos have toepads comprised of 'setae' which are stalk-like geometries which split off repeatedly into nano-scale geometries. The microscopic size of these setae creates intermolecular forces in the interaction of atoms between them and the surfaces they touch. The adhesion force between a singular seta and the adhesion surface is minuscule. However, there are approximately 14,400 setae per square millimetre on the toepads of the Tokay gecko - allowing it to support its body weigh with ease (Autumn, 2006). Recently, synthetic replications of the dry adhesion of geckos have been successfully created (Glick et al., 2018; L et al., 2019; Peressadko \& Gorb, 2004). Glick et al. (2018) showed how this can be used in harmony with soft robotics, by fabricating a soft robotic gripper with adhesive pads which increase the robot's gripping performance.

Wet adhesion utilises the surface tension of a liquid to stick to surfaces through static friction (Federle et al., 2002) and/or viscous forces (Hanna et al., 1991). A common example of this can be found in the Torrent frog, which secretes mucus from in between spongey toepads to allow for adhesion even on we surfaces been reproduced synthetically. Vogel et al. (2010) take inspiration from the wet adhesion of the leaf beetle to create a capillarity based switchable adhesion system which can pick up and drop substrates.

Mechanical interlocking is the interaction between solids to connect surfaces. A classic example of this can be found in Velcro (de Mestral, 1955) which was inspired by the hooks on burdock burrs which stuck to the clothes of de Mestral. Evidence for mechanical interlocking has been found in snakes in order to increase their grip when climbing trees (Hamidreza \& Hu, 2012). The scales on the underside of the snake's belly lock onto imperfections in the tree's bark, creating security if the muscular grip of the snake is to slip.

Suction forces create adhesion through a pressure differential between the internal cavity of the suction cup and the surrounding environment (Gu et al., 2016). In nature, this can be found in octopuses (Kier \& Smith, 2002) but suction cups have also been widely replicated synthetically for household and industrial applications.

\section{Muscular structure}

Muscular structure is a common source of biological inspiration for soft robots. A muscular hydrostat is a biological actuator primarily comprised of muscle tissue with no need for a supporting skeletal structure (Kier \& Smith, 1985). Muscular hydrostats are used by animals to interact with or manipulate elements in their surrounding environment. Muscular hydrostats are a commonly referenced form of biomimicry found in the field of soft robotics (Peele et al., 2015; Schaffner et al., 2018). This is because muscular hydrostats, like soft robots, can conform to their surroundings using only soft material. They are able to extend, twist, and bend by shortening and extending muscle fibres in specified directions or configurations to achieve their desired movement. This is reminiscent of the strain restriction in certain areas of soft robotic geometry which dictates the movement of the actuator. An importan distinction between muscular hydrostats and pneumatically actuated soft robots is that muscular hydrostats maintain a constan volume during actuation, and soft robots do not. 
EXPERIMENTATION 


\section{Introduction}

In response to $\operatorname{Aim}$ 2, this chapter demonstrates a range of soft robotic concepts that utilise the Stratasys J750 3D printer to explore the opportunities and limitations that it presents.

Adhesion testing was carried out to assess how Polyjet printed textures can enhance the capabilities of subsequent soft robotic concepts. They were first tested separately from the soft robotic prints to understand the success of the primary mechanisms of adhesion.

Biological inspiration, Software, Measurement, and Balloon Testing are all discussed prior to any pneumatically actuated soft robotic experiments performed on the Stratasys $\mathbf{J 7 5 0}$. This is used to detail the early design process; initial inspiration, CAD design, experimentation setup, testing fundamental concepts of actuation.
The soft robotic inflation tests use the prior information covered in this chapter to directly tackle Aim 2. This details the range of concepts that were tested, all presented in chronological order These were tested with a small air pump with a maximum capacity of 10psi. A pressure sensor was used to relay data back to an Arduino, which displayed this data on a connected laptop.

The naming convention used to identify the prints is as follows:

1.01 - 1.14: Extension geometry actuators

2.01 - 2.10: Bending geometry actuators 3.01 - 3.17: Twisting geometry actuators

4.01 - 4.08: Expansion geometry actuators

5.01 - 5.07: Complex motion actuators

\section{Stratasys 1750}

The Stratasys J750 is the printer that was used for the primary testing of this portfolio. The printer has a build size of $490 \times 390 \times 200 \mathrm{~mm}$ and a horizontal build layer thickness of 14 microns. The materials that were used are AgilusClear and Vero. AgilusClear (Agilus) is the soft material resin loaded in the printer, and it has a minimum shore hardness $\mathrm{SH}$ of 30 (30SH). The Vero material resin came in four colour variations; cyan, yellow, magenta, and black - all with $95 \mathrm{SH}$. These can be combined in different ratios to support a range of possible shore hardness, shown in Figure 5. This was fully utilised throughout the experimentation to explore a range of material shore hardness combinations.

The Stratasys $J 750$ uses a support material, called SUP706, to build models with overhanging geometry. After printing, this needs to be removed. A chemical solution bath was used to remove the support material which could not be manually removed with tools. This solution consisted of $2 \%$ sodium hydroxide and $1 \%$ sodium metasilicate, and a pump which circulates the solution. The prints in this portfolio were kept in this bath for 2-4 hours, before being removed from the bath and rinsed off.

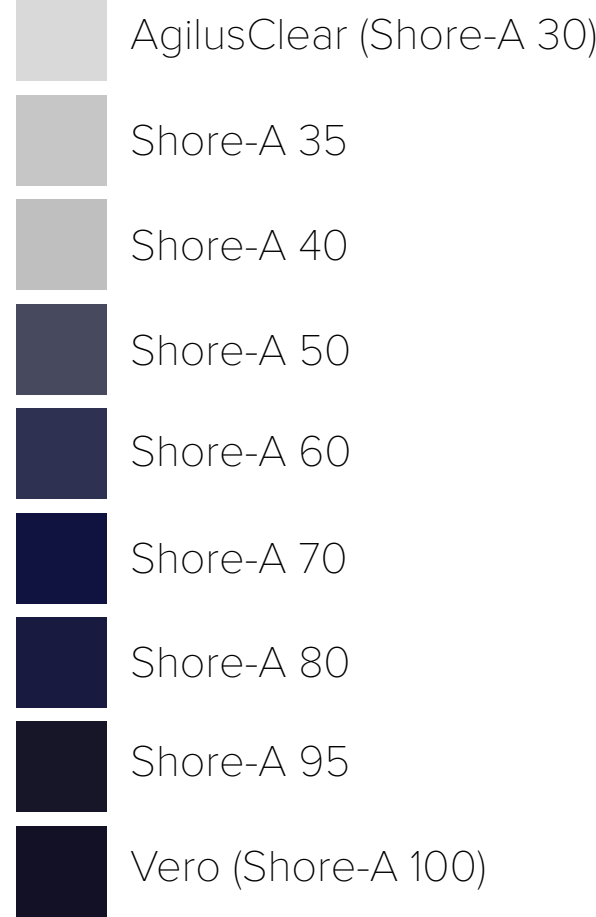

Figure 5. Stratasys J750 Shore A Hardness printable materials. 


\section{$\underline{\text { Adhesion testing }}$}

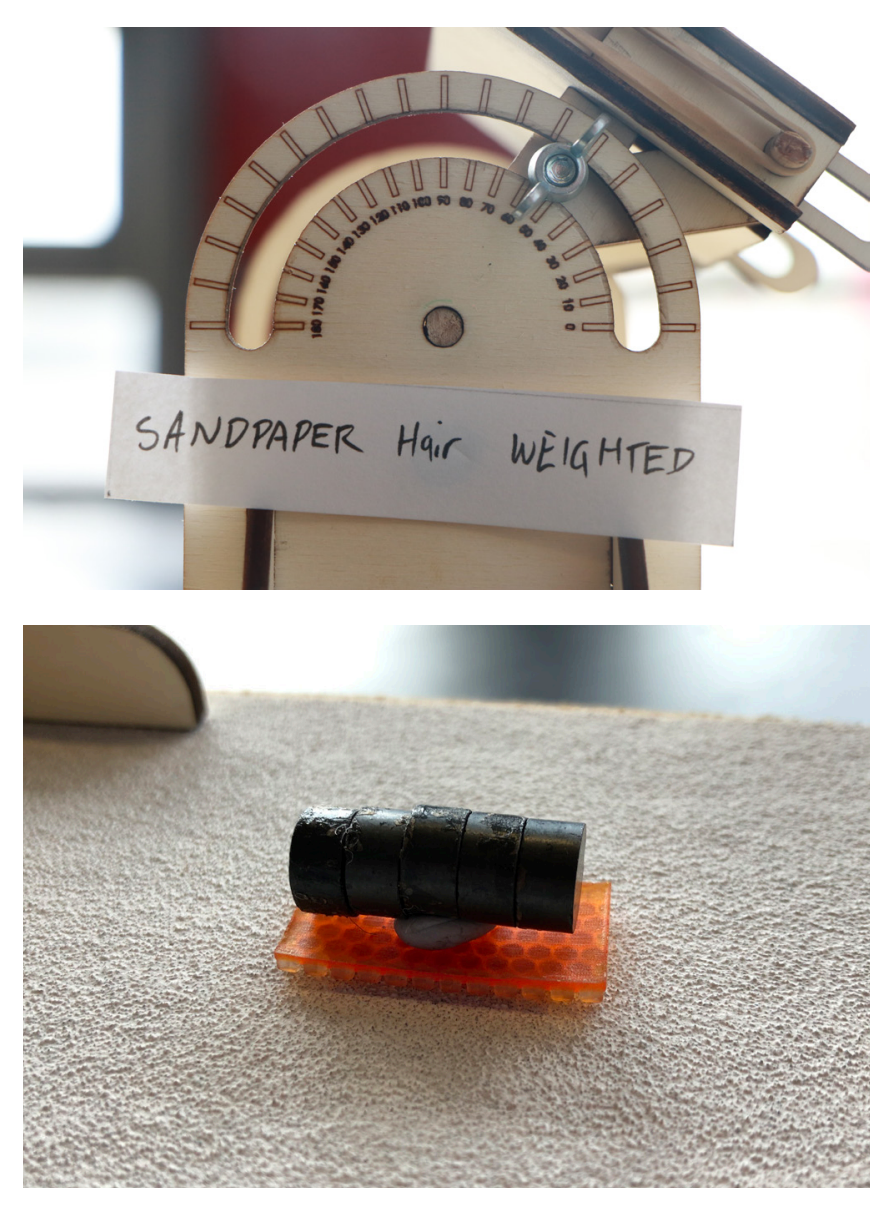

\section{Goals}

Figure 6. Adhesion testing setup.

A selection of natural adhesion mechanisms were plate could be secured, and its angle changed. The mimicked and printed on the Stratasys J750. These materials that were tested were:

included:

\section{MDF (smooth) \\ Sandpaper (coarse) \\ - Felt (soft)}

Gecko toepads

Frog toepads

Adhesive hairs

Suction cups

Snake scales

To test the angle of failure of each texture, the angle of the material plate was gradually increased from $0^{\circ}$ until the texture fell off the material plate These prints all focused on either dry adhesion, where the value was recorded. Each texture was mechanical interlocking, or suction. Wet adhesion also tested with a small weight added, to see if that was not focused on because it would require an would impact the performance of each texture. As additional human intervention to implement the a control, a smooth pad of the 30SH Agilus material adhesive liquid. To test the effectiveness of these was printed. Each pad was $30 \mathrm{~mm} \times 15 \mathrm{~mm}$ in size. prints, a rig was assembled in which a material 

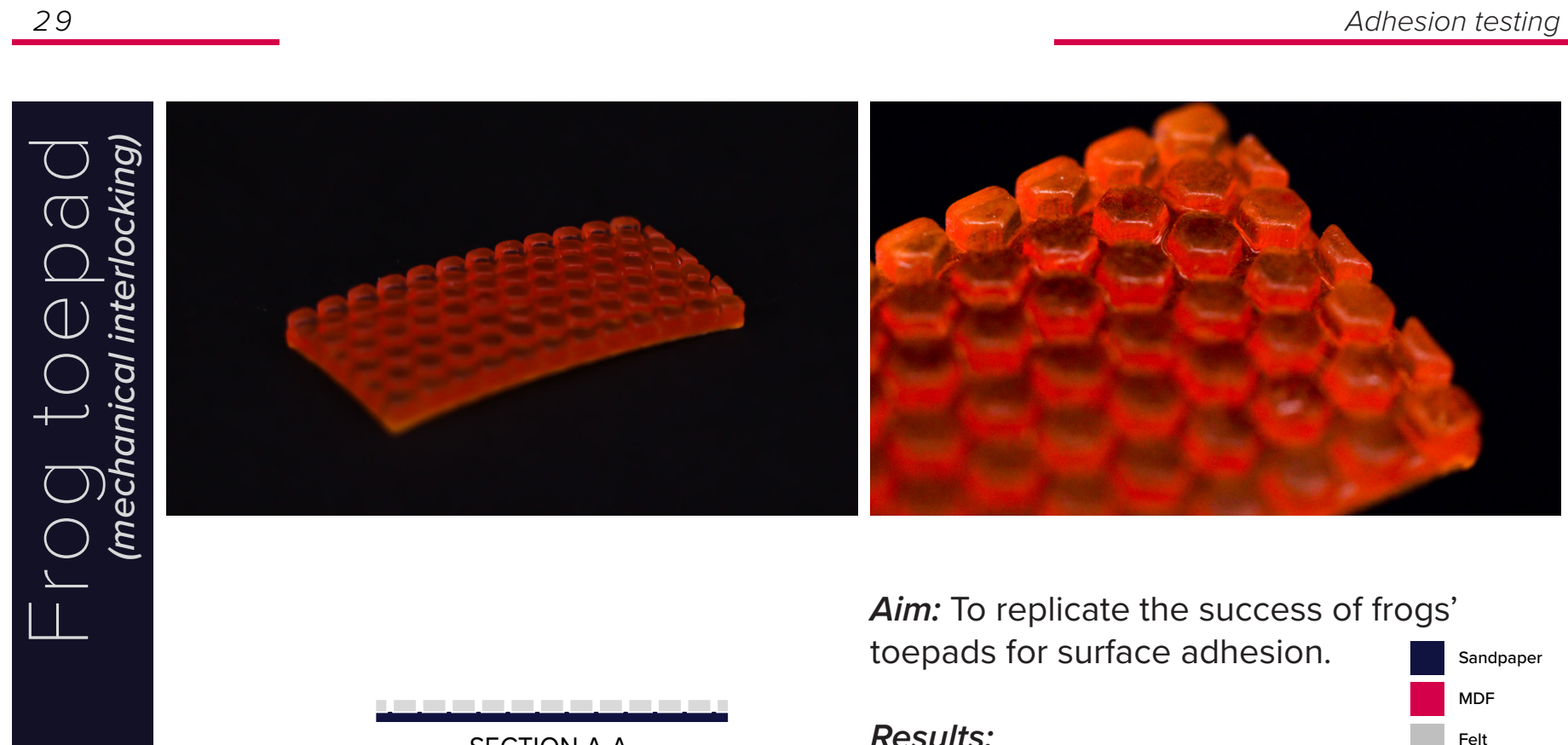

Aim: To replicate the success of frogs'
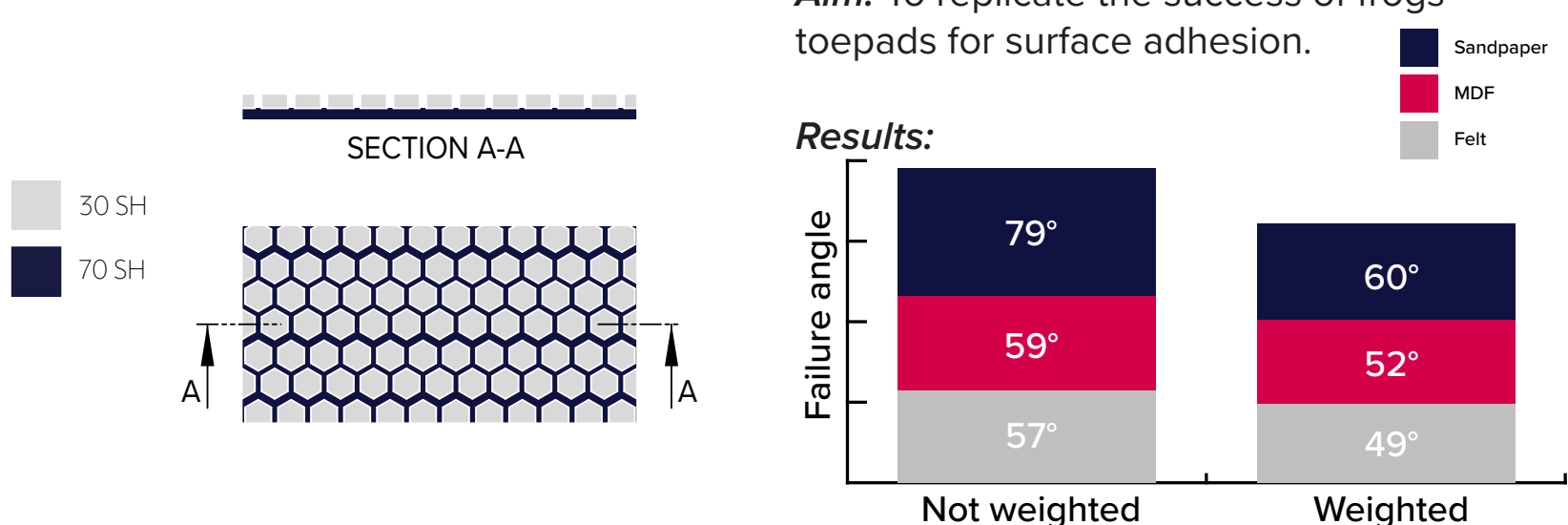

Figure $7 a-7 d$. Frog toepad images and results.
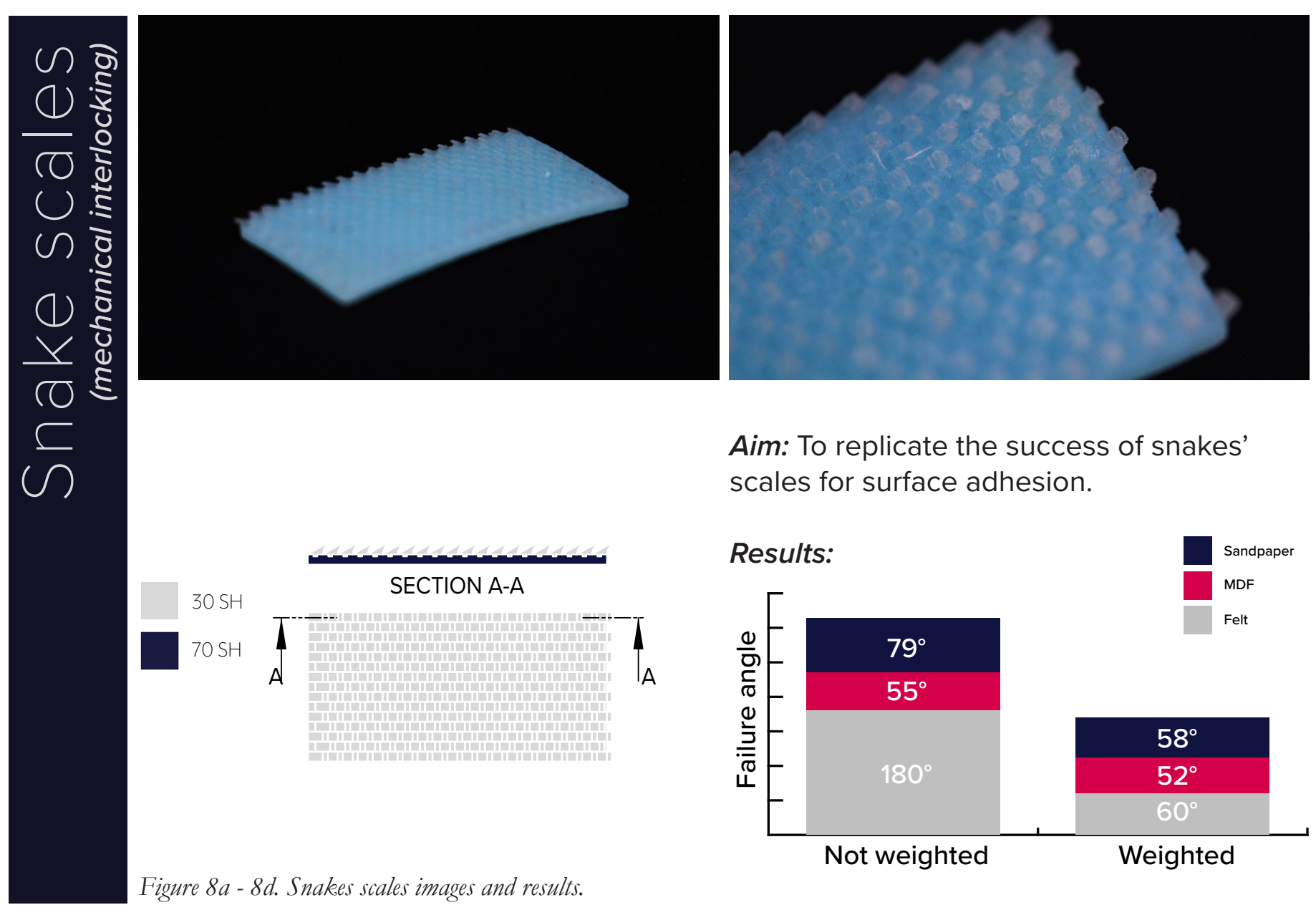

Aim: To replicate the success of snakes scales for surface adhesion.
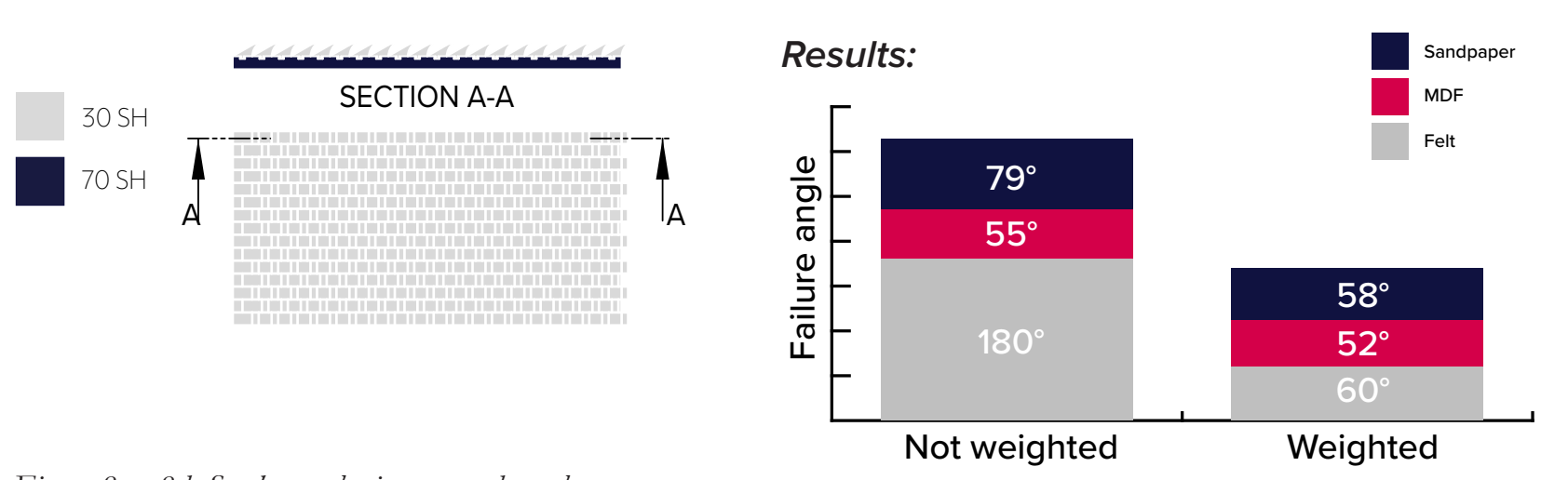

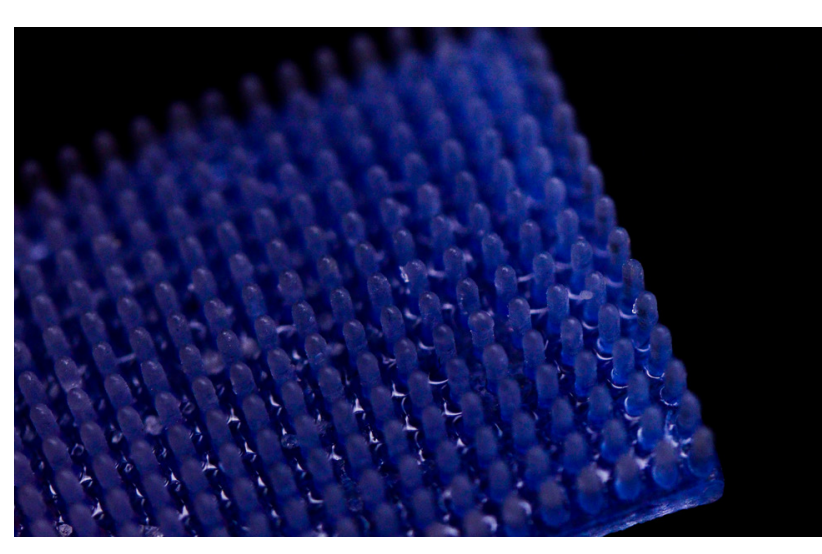

Aim: To create adhesive hairs for surface adhesion.

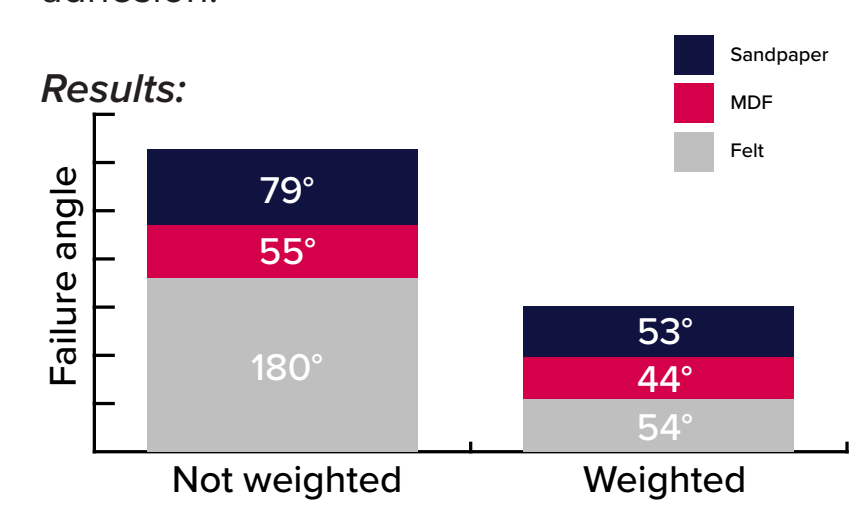

Figure 9a - 9d. Adhesive hairs images and results.
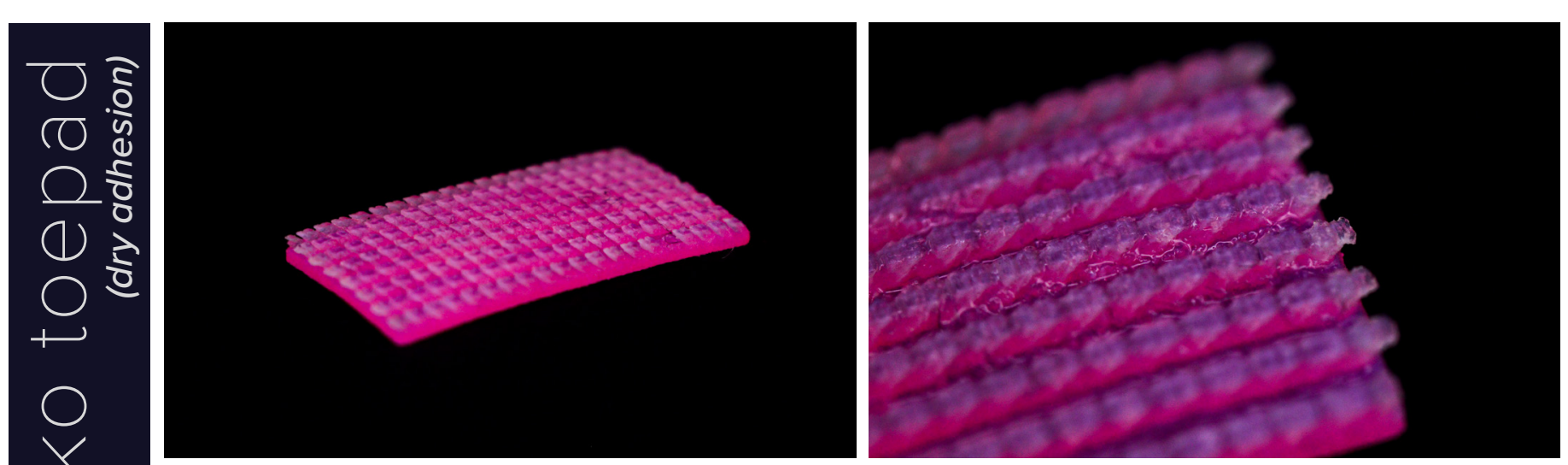

$\frac{V}{U}$
0

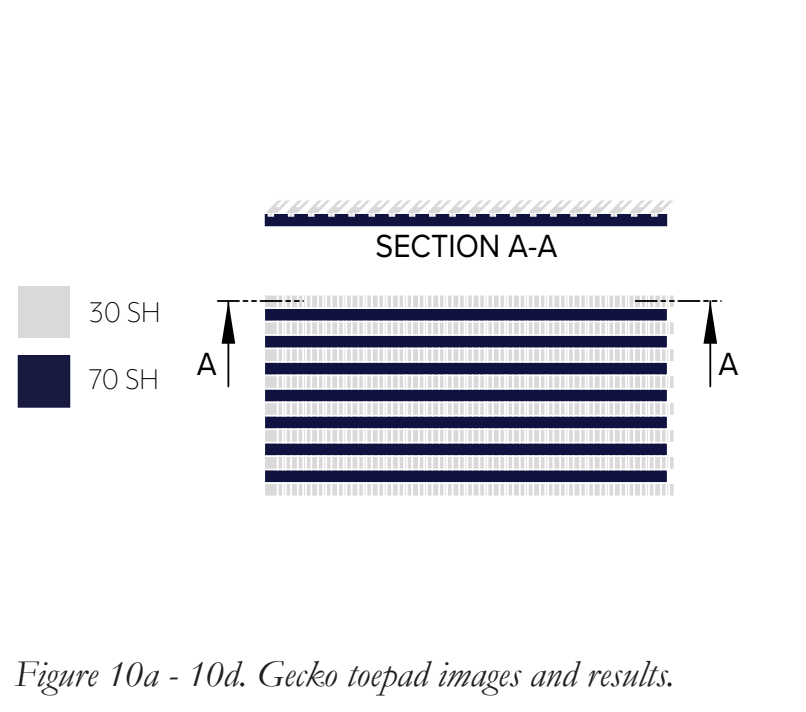

Aim: To replicate the success of geckos toepads for surface adhesion.

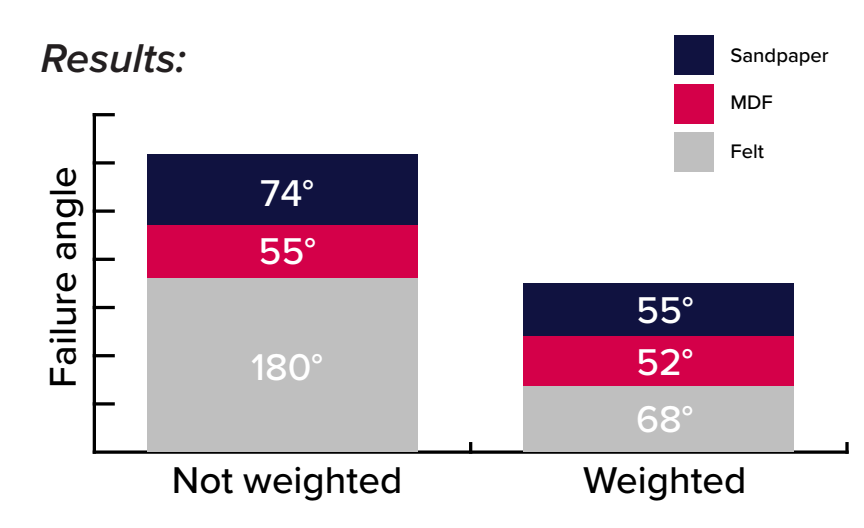



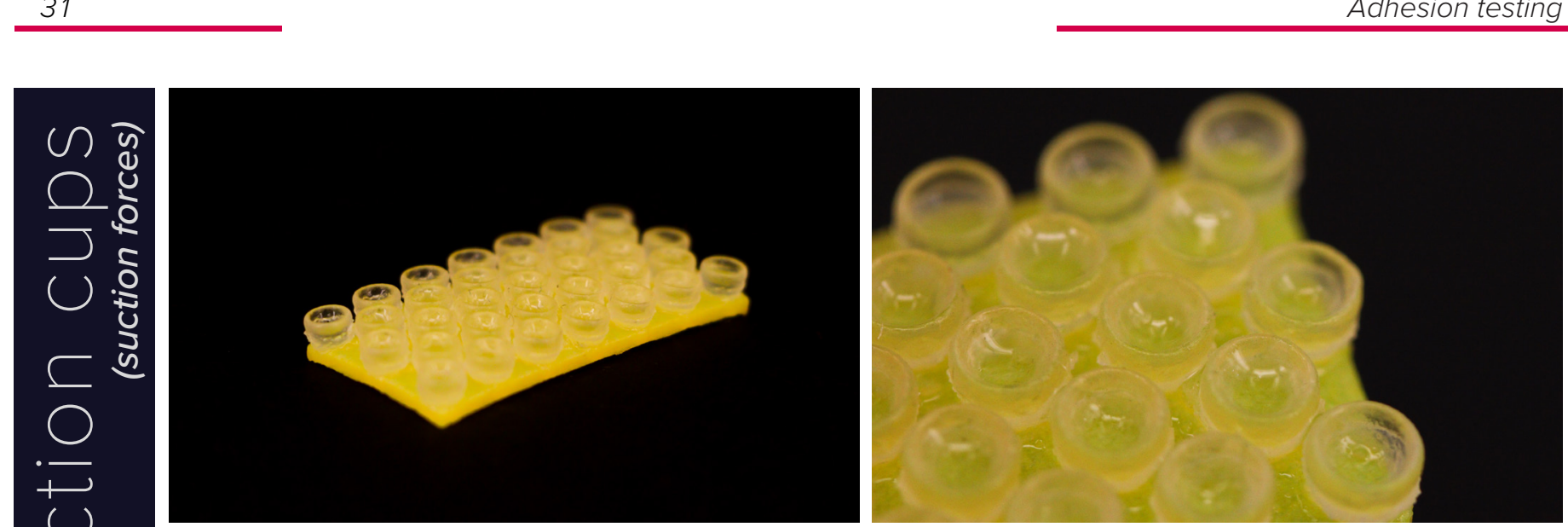

Aim: To replicate the success of octopuses suction cups for surface adhesion.

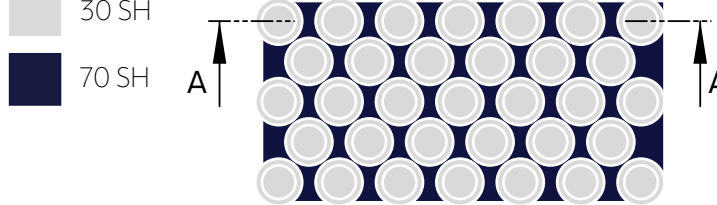
0101919101

Results. The suction cup was not tested using the same setup as the other prints as its adhesion was reliant on a localised pressure being applied to press it against a surface. The cups successfully allowed for the pad to be connected to surfaces, however, the surfaces needed to be very smooth for this to work.

\section{Analysis}

The maximum adhesion was observed on the felt material - the snake, gecko, and adhesive hair textures all remained attached to the felt when upside down. However, once weight was added, the failure angle reduced significantly for all tests. The control Agilus pad consistently performed the worst in all scenarios, validating the added performance of adhesive geometry. All of the adhesion geometries were printed as small as physically possible within the printable resolution icated a scale where Van der Waal forces would act as the primary anical interlocking properties still provided an increase in adhesive performance.

Adhesion decreased significantly on the sandpaper and MDF. This is likely due to the successful adhesive pads relying on mechanica interlocking; if the surface texture was too smooth for interlocking, the adhesion mechanism functionality was diminished.

As mechanical interlocking was the method of adhesion that was most successful, this was replicated and reintroduced to assist with locomotion in subsequent soft robot concepts. 


\section{Biological inspiration}

Muscular hydrostats were used as inspiration to inform the initial designs. Examples of extension, bending, and twisting were examined at a muscular level, and these were then adapted for pneumatic soft robots (Figure 12). These three motions were chosen as clear examples could be found throughout nature, and they were each distinctly different and would each require different geometries to successfully mimic the motion.
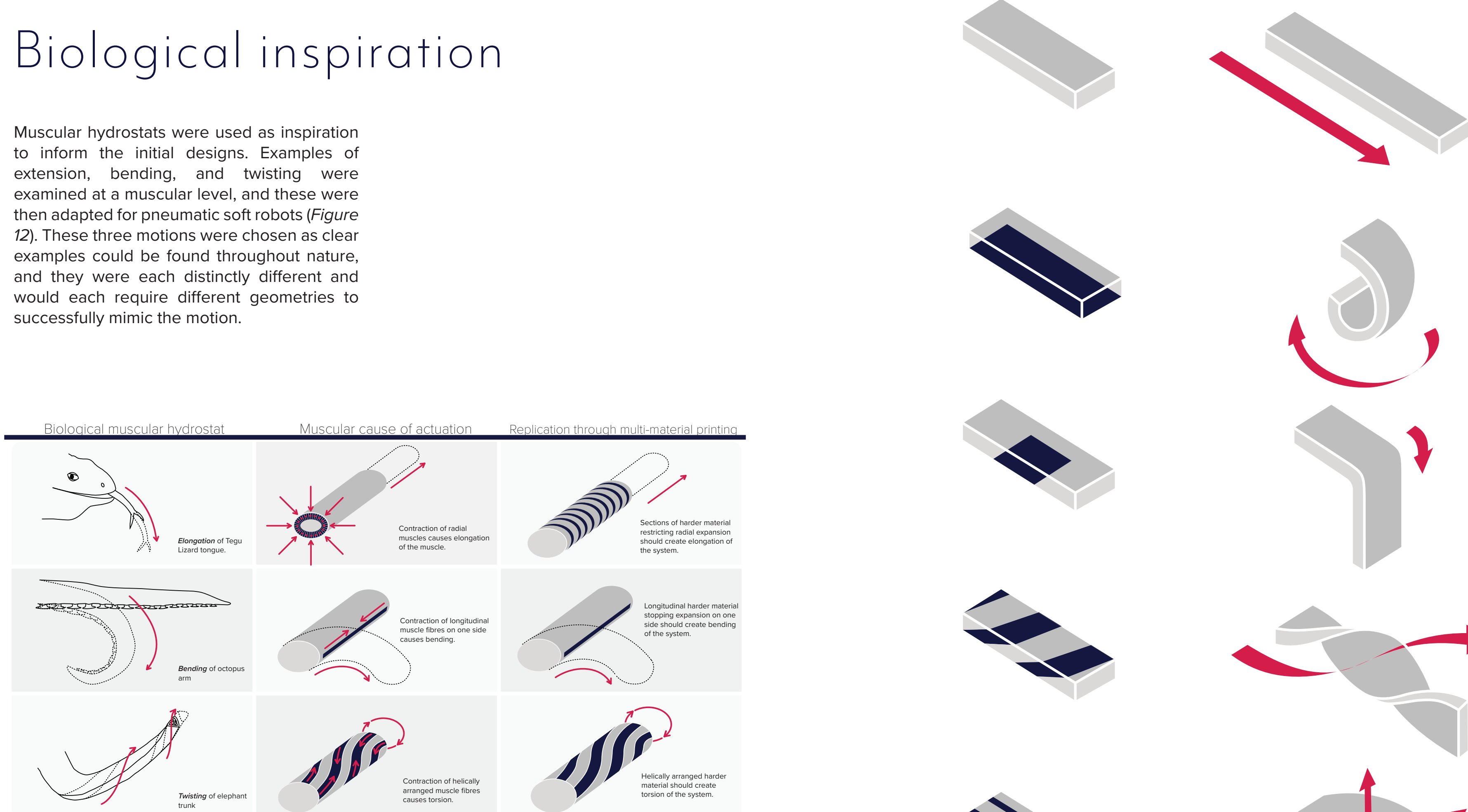

ts and method for printed replication.
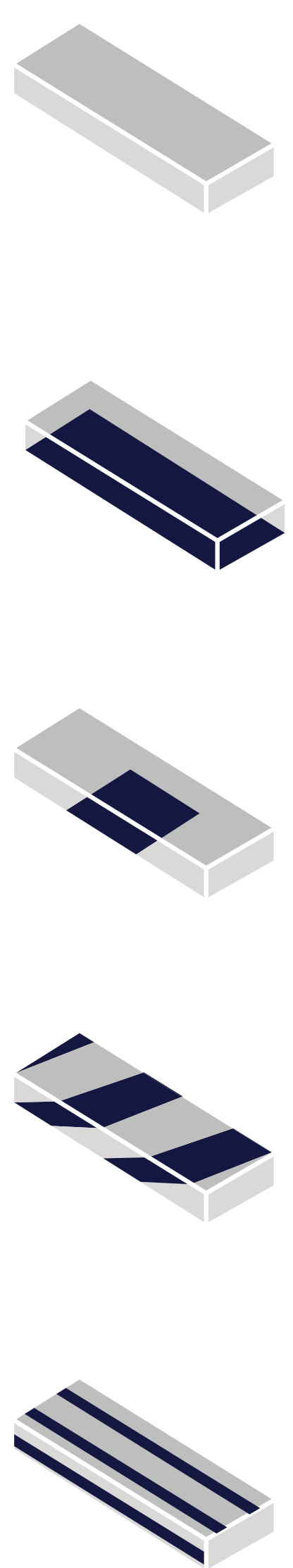

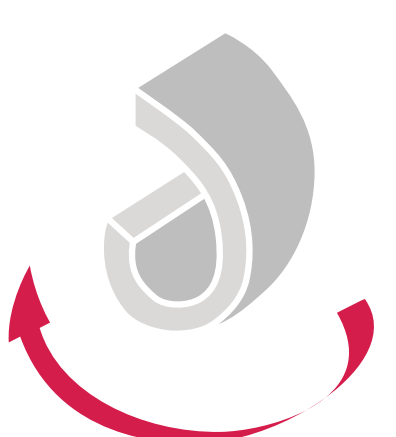

1

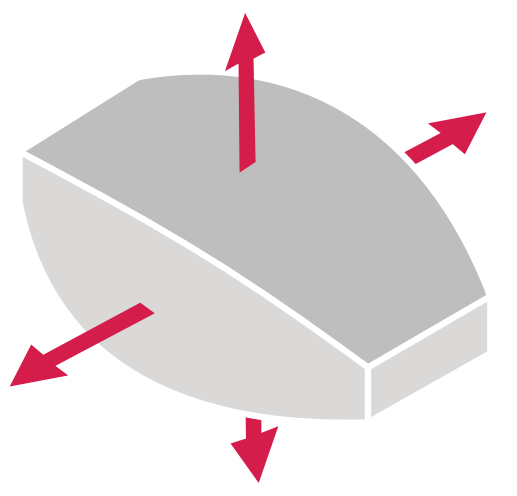




\section{Software}

To achieve a blueprint for a robust soft robot that can be applied to a wide variety of scenarios, it was vital to create a system where the actuators can be easily altered to perform specific tasks. This has been tackled by using equation driven models in Solidworks that allow the model geometry to adapt when a parameter is changed. Global variables were assigned to the driving parameters, which other dimensions were referred to, which allowed these dimensions to vary in accordance with changes made to the global variables. This system was used to not only create model flexibility when designing for scenarios, but also to streamline the testing and iteration processes. As the experimentation in this research looked to find optimal performance in geometry and materiality, the equation driven models allowed for quick and easy iterations. For example, the helix angle of the twisting actuator was be given multiple values, and separate models were created for each value of helix angle which were 3D printed. The prints were then tested, compared, and the optimal helix angle was determined.

Every actuator was derived from a singular base geometry. A set of curves was defined to build the bellows off. These curves were then repeated in a pattern and rotated around a central axis to create the geometry. To achieve different motions from the actuator, alterations were made from this point to modify the output motion from the actuator. The extending motion geometry operates solely off the base geometry; however, modifications were made to the twisting and bending geometries to achieve the desired motion.

To create a bending motion, a radial asymmetry needed to be incorporated as informed by a number of precedents across the literature (llievski et al., 2011; Martinez et al., 2013 Peele et al., 2015; Hong Kai Yap et al., 2016). This asymmetry could be achieved through geometry or materiality, both of which were tested. To incorporate a degree of twist into the bending motion, the chamber angle was changed so, although the chambers were still patterned along the same axis, they did no remain perpendicular to the axis.

To achieve a twisting motion, a helical element needed to be incorporated (Schaffner et al., 2018), once again, either through geometry or materiality. To explore a twisting motion through geometry, the base geometry was patterned radially rather than axially, and that profile was extruded with a twist angle implemented. As an extension of this model, when the twist angle was set to $0^{\circ}$, an expansion motion could be achieved through actuation.

\section{Equation driven models}

To increase the control over the CAD models, a series of equations were derived. These rely on a set of global variables to drive them. For example:

In the twisting actuator, the twist was defined using the "sweep" feature in Solidworks. The "sweep" feature allows you to enter a value for the profile twist or "sweep angle" which is the total angle of twist over the predetermined length. To build this model, it was importan that, if the length of the twisting actuator was changed, the pitch would remain the same. An equation needed to be used to drive the "sweep angle" such that this was always the case. The model's twist was driven by the global variable thelix angle which describes the angle of a helix as a straight line that runs tangentially to the helix from the central axis. Equations for the "pitch" and the "sweep angle" were used to allow the model to be driven by a change in value for the "helix angle".

$$
\begin{aligned}
& \text { "pitch" }=\frac{2 \pi r}{\tan (" \text { helix angle") }} \\
& \text { "sweep angle" }=\frac{360 \times \text { "length" }}{\text { "pitch" }}
\end{aligned}
$$

These equations allow for the length of the twist ("length") to be changed while the angle of the twist ("sweep angle" as defined in the Solidworks "sweep" feature control) remains the same. The "sweep angle" can also be altered through the "helix angle" global variable.

Equations were used to achieve similar results in the other actuators where dimensions and the number of bellows that were patterned had global variables assigned that could easily be changed for iterative and applications purposes.

\section{Base geometry design and parameter control}

The base geometry is derived from a series of bellows patterned along a central axis. The driving parameters can be found in Figure 14.

It was important to avoid right angles or sharp corners in the internal geometry of the bellows, as this can create areas of weakness which are prone to tearing.

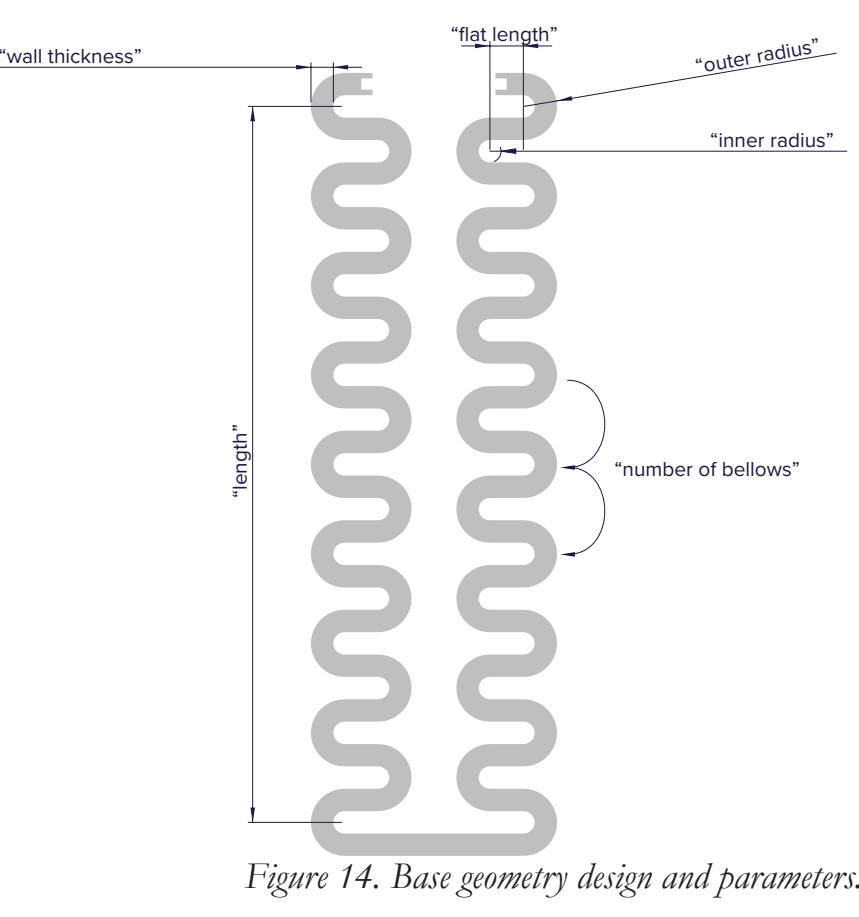

\section{Modularity}

The equation driven model design allowed for modularity of complex actuators. When multiple movements were combined into the same Solidworks assembly, they could be simply connected in any configuration using a connection piece. This allowed for an efficien design workflow - after the original Solidworks models had been created, virtually no more time needed to be spent on modelling as the models could be configured individually with an input piece or configured with other sections in an assembly. Once the pieces are mated in an assembly, the individual parts can still be edited and the changes updated in the assembly. For example, if a previous mode exhibited a higher bend angle than was wanted in an experiment, the number of bellows could be decreased at a part level and the assembly would automatically change to accommodate this as shown in Figure 15. 


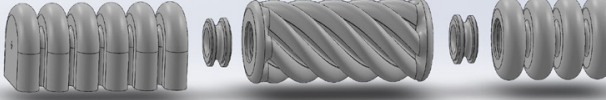
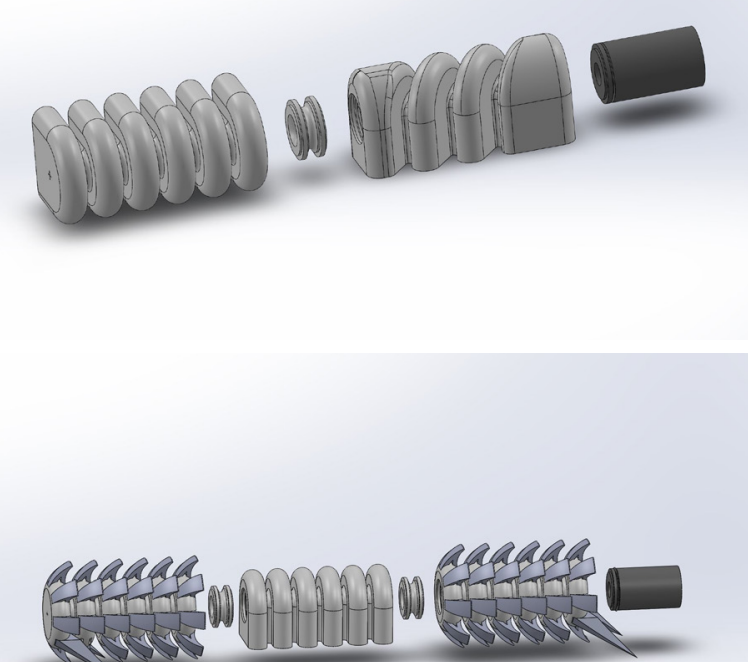

In order to accurately quantify the performance of different actuators, individualised recording and testing methods were created (Figure 16). The actuators that had their performance quantified were:
Bending
- Twisting
- Extension
- Expansion

They were each recorded on video with the actuator positioned in the best orientation to demonstrate its deformation. Afte actuation, the footage was reviewed in Adobe Premiere Pro, and video frames that showed the initial position of the actuator and the actuator in its deformed state were exported. Adobe Illustrator was used to measure the Adobe Illustrator was used to measure the
deformation, through the "Info" window which deformation, through the "Info" window which

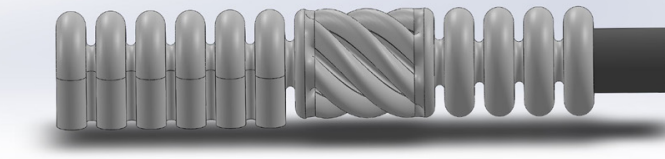

HAN Nown

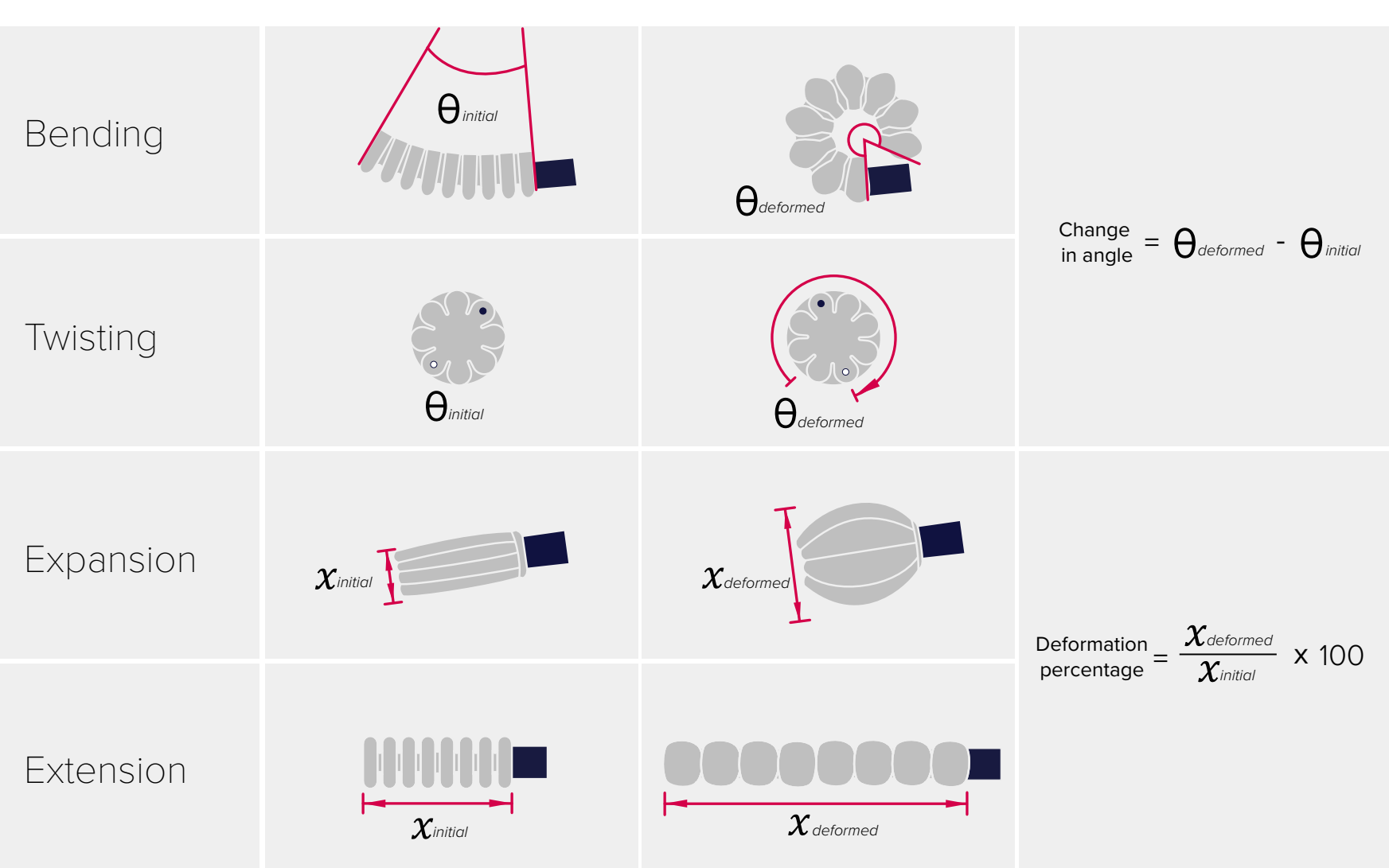

Figure 16. Method for measuring each primary inflation motion. 


\section{Balloon testing}

To test the basic concepts of actuation for soft robots, bend was created in the balloon during inflation. This testing was carried out through the use of balloons method allowed for precise control over where the and tape (Figure 17). Using this method, principles of bending occurred along the length of the balloon, strain restricting regions to control motion as found as is demonstrated by localising the bending in the in biology and synthetic replications could easily be orange balloon.

tested and inflated without the need for designing

or assembling of a testing rig. Electrical tape acted In muscular hydrostats, a contraction of helically as the strain restrictor as the balloon was inflated. arranged muscle fibres creates torsion along the These principles could be heavily inspired by the system. To replicate this with a balloon and tape, muscular systems found in muscular hydrostats. A the tape was wrapped around the balloon helically key distinction with muscular hydrostats is that they prior to inflation. This also proved to be successful maintain a constant volume at all times and thus, in proving the concept, however, it was found that the system for actuation must be adapted to suit the helix angle of the tape had to be very specific a change in volume during actuation. Instead of a in order to fully inflate, otherwise the balloon would muscular contraction occurring to dictate motion not inflate past the beginning of the length of tape. muscular contraction occurring to dictate motion
like in muscular hydrostats, inflating actuators have a region which maintains its dimensions while others The main drawbacks with taking this system expand around it. By placing the electrical tape in any further was the expansion of the balloon differing configurations on the balloon prior to its dimensions after inflation. The surface area of the inflation, it was found that the balloon would follow balloon expanded so significantly that it proved to be a different path during inflation. Both bending and challenging to place the tape in certain orientations twisting were tested with tape, however, extension without the balloon bursting. As the tape expanded occurred once the balloon was inflated without any very little during inflation, a large stress was created tape needed so this wasn't tested.

from the axial asymmetry of expansion.

In muscular hydrostats, a contraction of longitudinal These tests not only worked to prove the driving muscles on one side of the hydrostats causes a principles informing future design decisions, but bending motion to occur. To replicate this with they also informed the system for parametric balloon and tape, a strip of tape was placed control. When the balloons were deflated, tap longitudinally along the balloon to restrict expansion could be placed in any location along their length to along one side. This worked as anticipated, and a inform the motion during inflation.

Bending
Localised
bending

Figure 17. Balloon testing experiments.

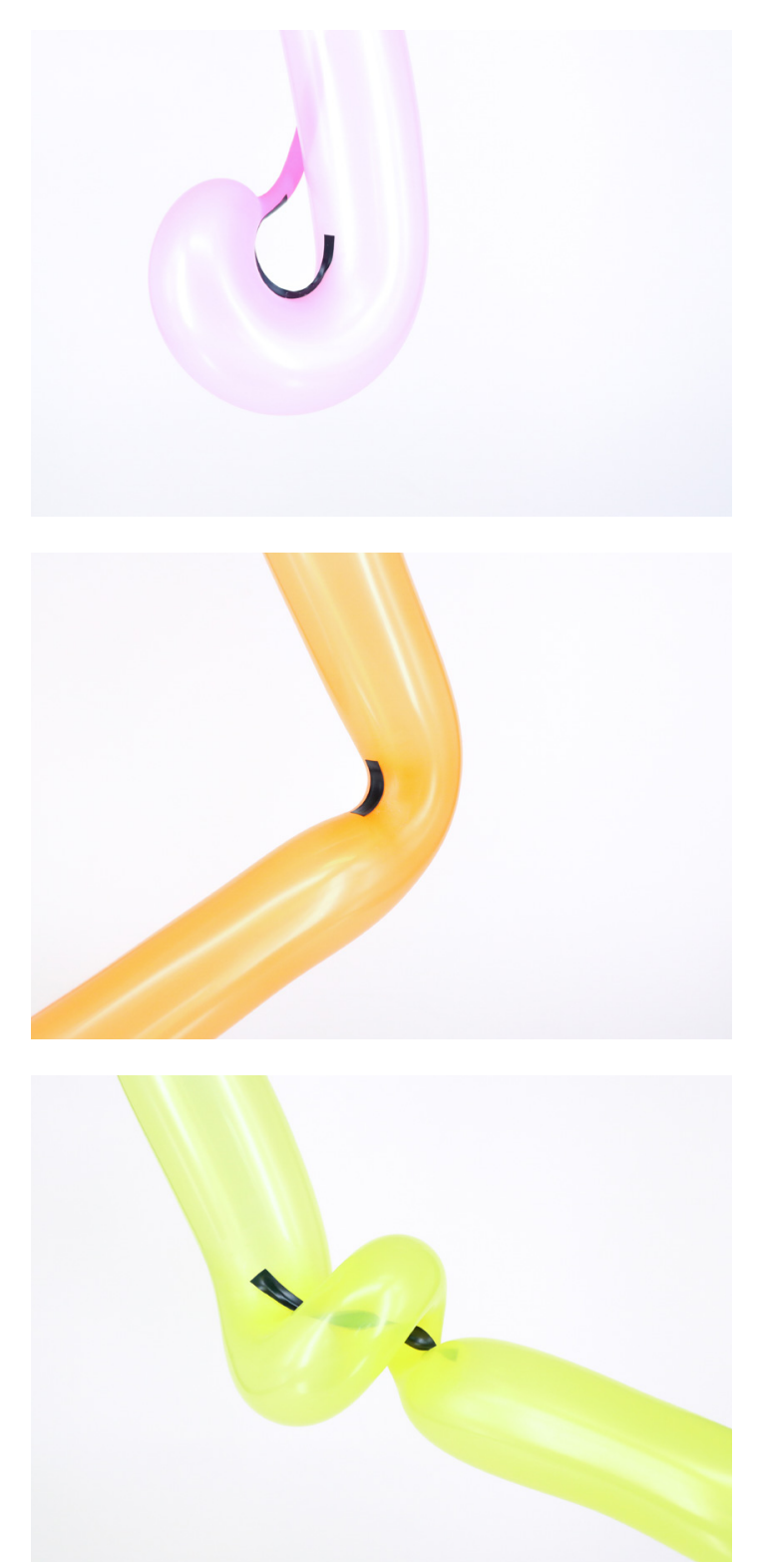




\section{Print set 1}

\section{Goals}

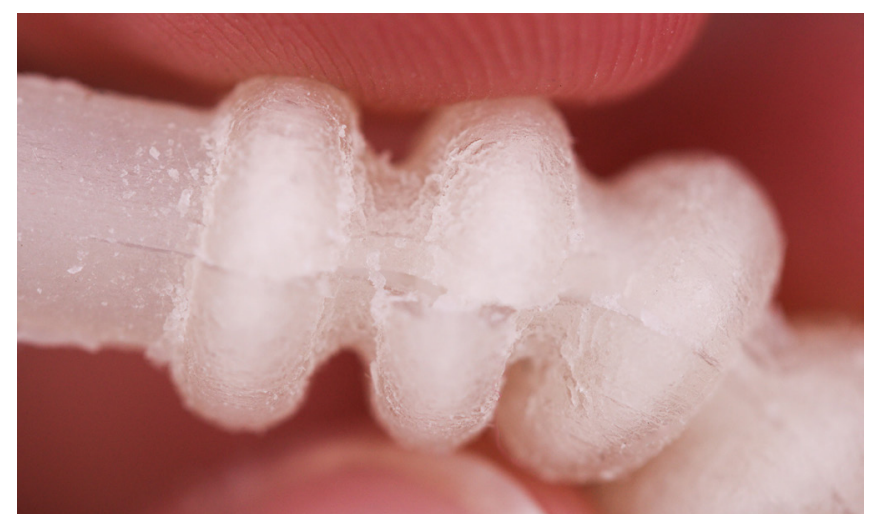

The goal for this print set was to explore a singular base geometry which could be modified to achieve three different motions. These motions were:

- Extension

Bending

Twisting

This print set worked as a first test to analyse the strength of the material, assess the wall thickness and shore hardness, and begin to establish a technique and process for cleaning the support technique and process for cleaning the support
material from the models and preparation for inflation. 


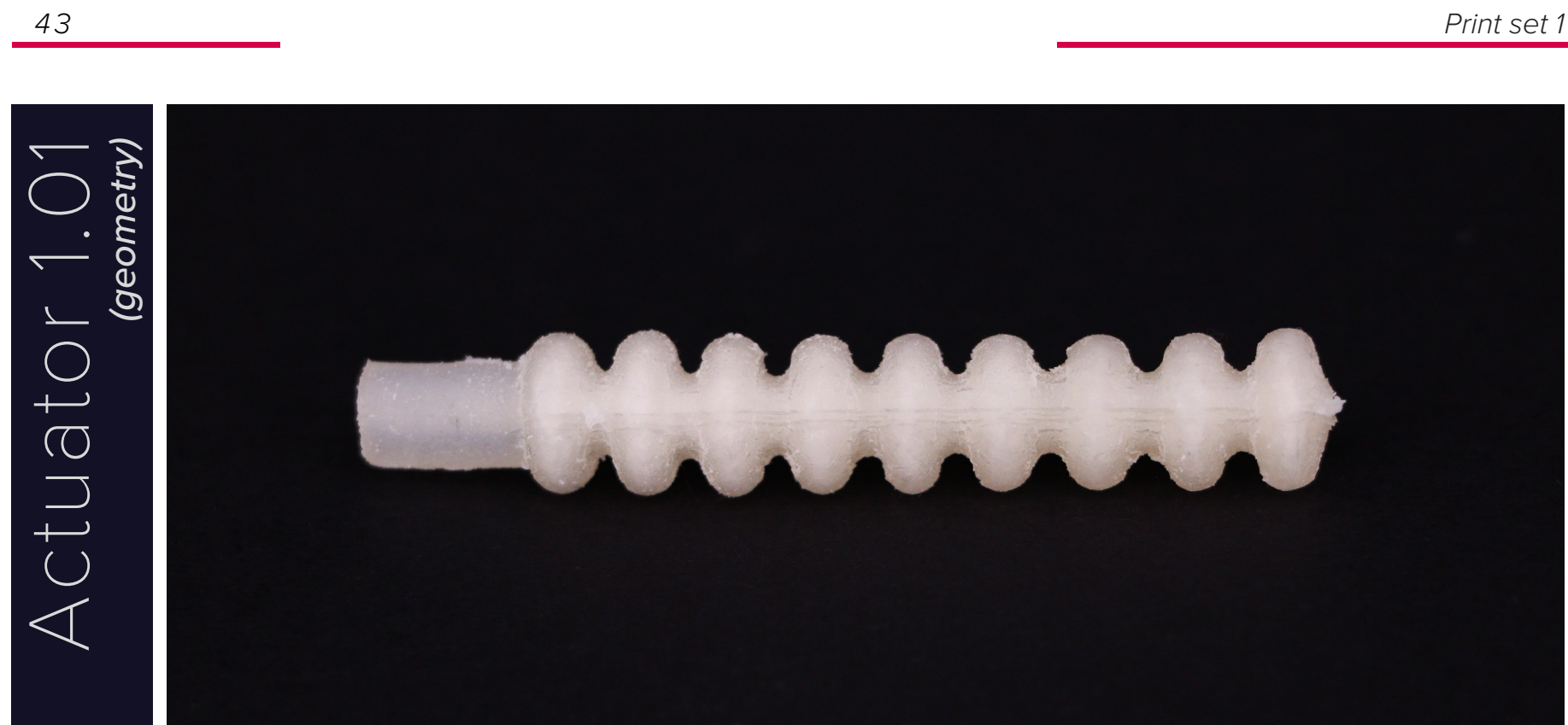

Aim: Create a soft actuator capable of extending when inflated.

30sH Results: Tears occurred during the - $50 \mathrm{SH}$ cleaning of support material from the print.

Figure 18a-18b. Actuator 1.01 images.

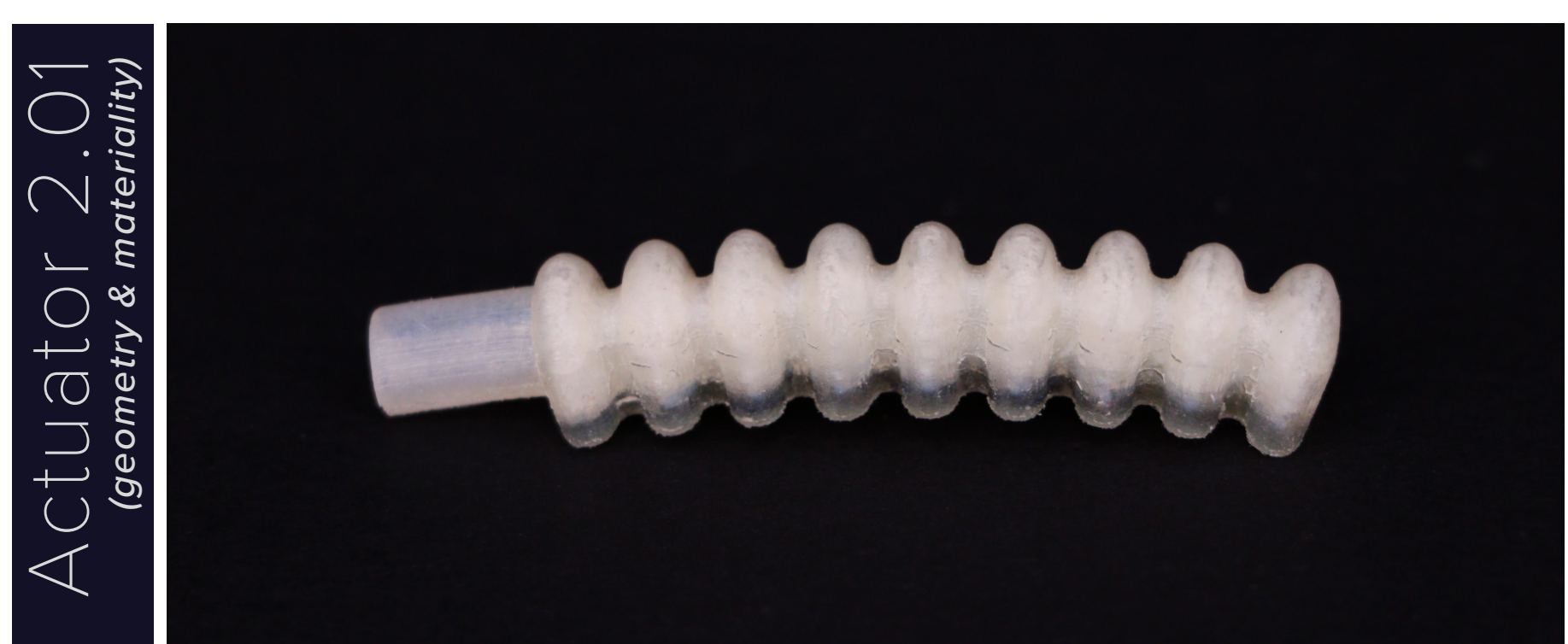

Aim: Create a soft actuator capable of bending when inflated.

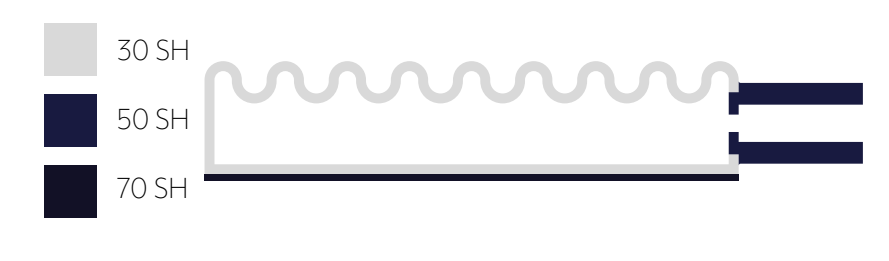

ults: Tears occurred during the cleaning of support material from the print. Delamination occurred between the 70SH material on the base and the main soft body.

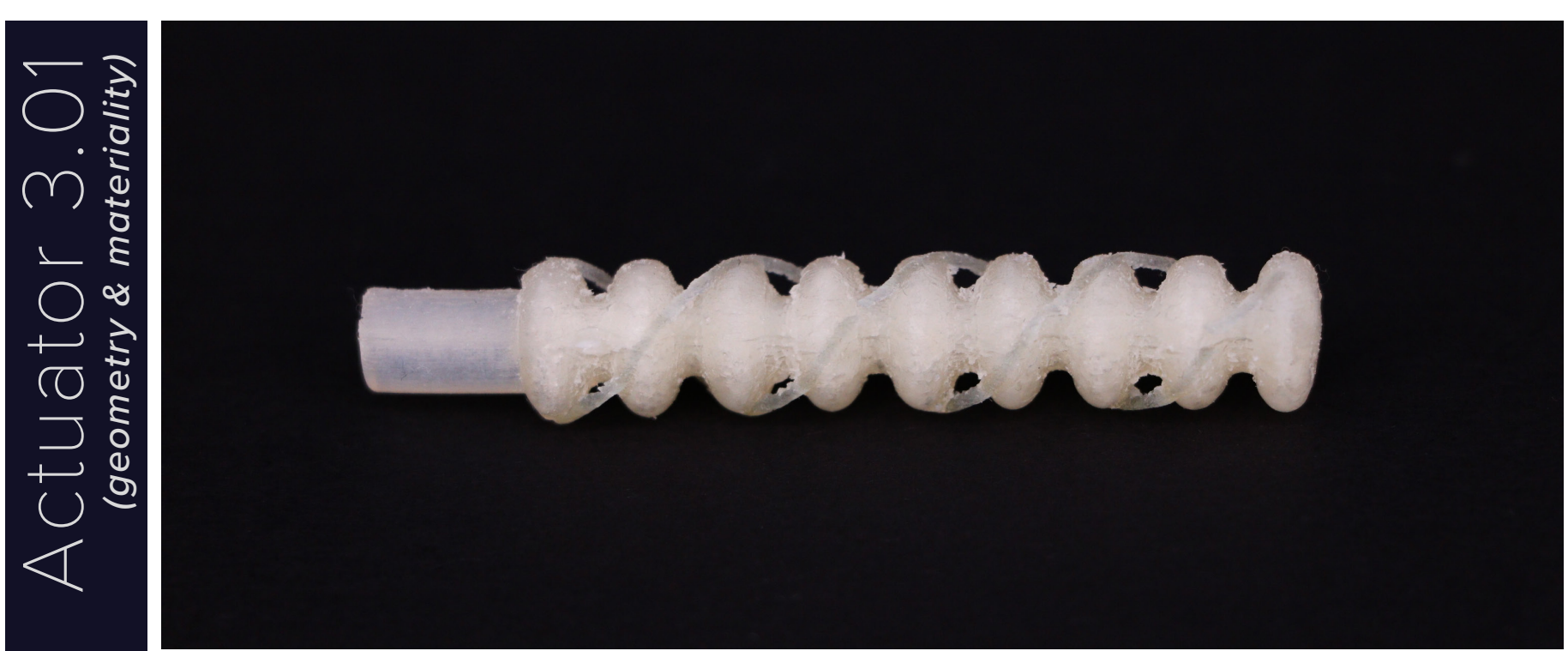

Aim: Create a soft actuator capable of twisting when inflated.

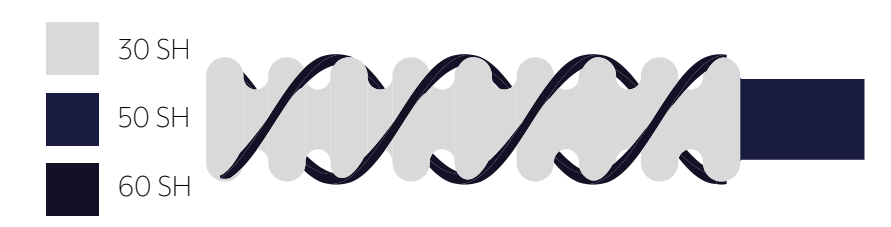

Results: Tears occurred during the cleaning of support material from the print. The helically arranged $60 \mathrm{SH}$ material was too thin and broke easily. 


\section{Analysis}

As shown in Figure 21, the prints in this set all failed

due to tearing before they could be inflated and

the motion could be tested. As the models were so

prone to tearing subsequent designs needed to be

much stronger to survive cleaning and especially to

much strong to sul

perform well once inflated. A cleaning process was

also be established to minimise the damage done

to the prints, and to standardise the procedure

across print sets. 
Print set 2

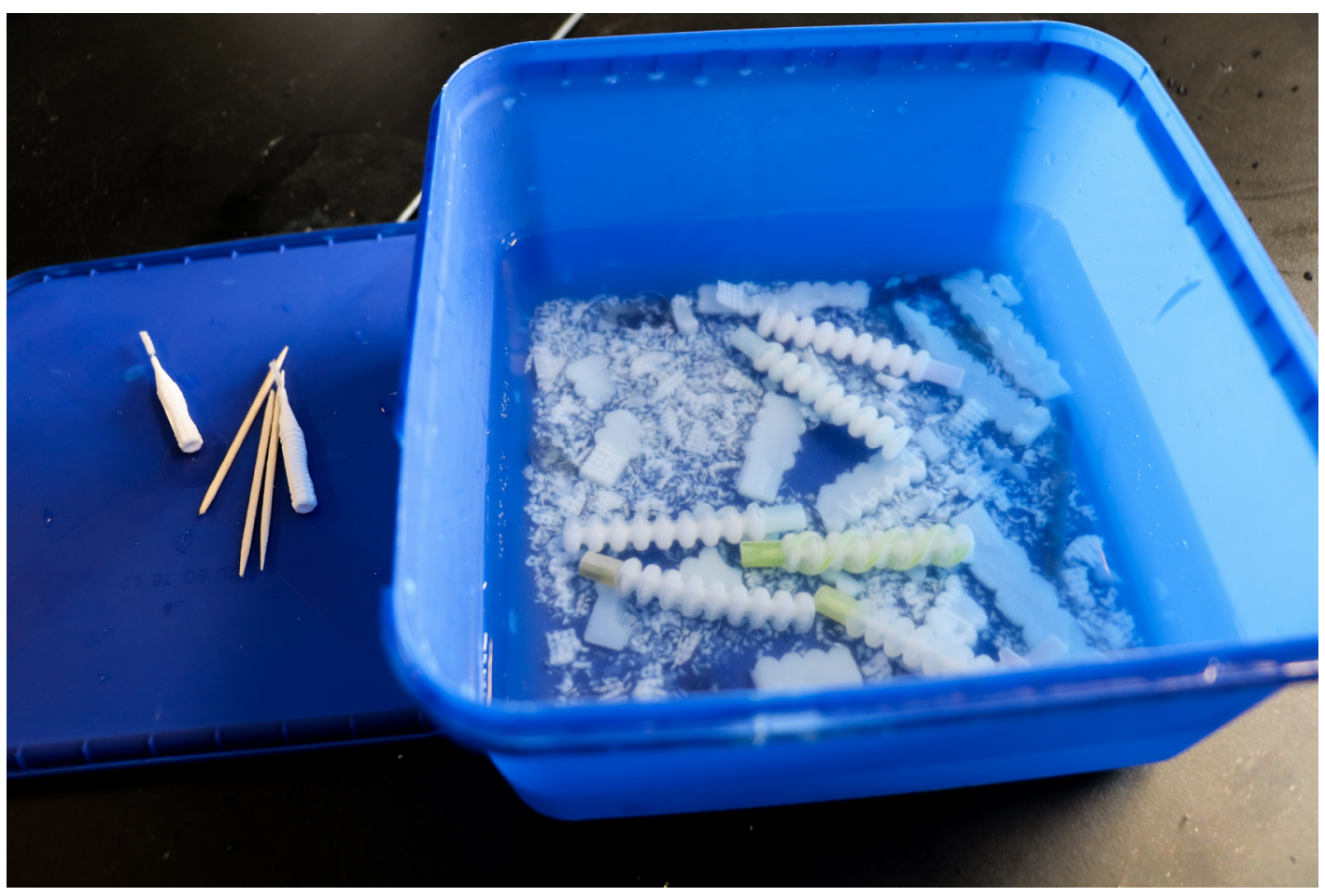

Figure 22. Support material cleaning setup.

\section{Goals}

This print set aimed to explore the success of the performance of actuators by utilising different properties of the 3D printer. These included actuators designed around:

\section{- Materiality and geometry}

Materiality

Internal geometry

Extension, twisting, and bending were evaluated for each of these routes. All of the actuators were printed with a glossy finish on them.

To improve on the previous models the following changes were made:

Increased chamber wall thickness $(0.8 \mathrm{~mm}$ $1.0 \mathrm{~mm})$

Interlocking geometry at the connection between the main soft body and the input geometry to reduce the chance of tearing.

Increased main soft body shore hardness ( $\mathrm{SH} 30$-> SH35).

- Hard material areas in the bending and twisting actuators were embedded within the main soft body to reduce the chance of separation between bodies.

A relief valve was added to the end of the chambers. This was to help with the removal of support material from internal chambers and to reduce the chances of bursting at high actuation pressure. This also altered the actuation pressure needed as the new system needed a constant input of air.

To improve the cleaning process of the models, the following workflow was implemented:

\section{. CAD modelling}

2. Printing

3. Soak prints in water

Rough removal of support material while in a water bath (Figure 22)

5. Solution bath

6. Finer detail support material removal and solution rinsing while in the water bath

7. Washing/drying

8. Glueing relief valves closed

9. Pressurisation

Pressurisation is used as the final step in the postprocessing of the actuators as the flow of air helps to loosen the remainder of the support materia from the internal chambers of the actuators. The rom air incut. The valves were then closed off with The valus we super glue to make the system airtight to decrease the volume of air needed for actuation. Actuation results were taken before and after the valves were sealed to test this hypothesis. 

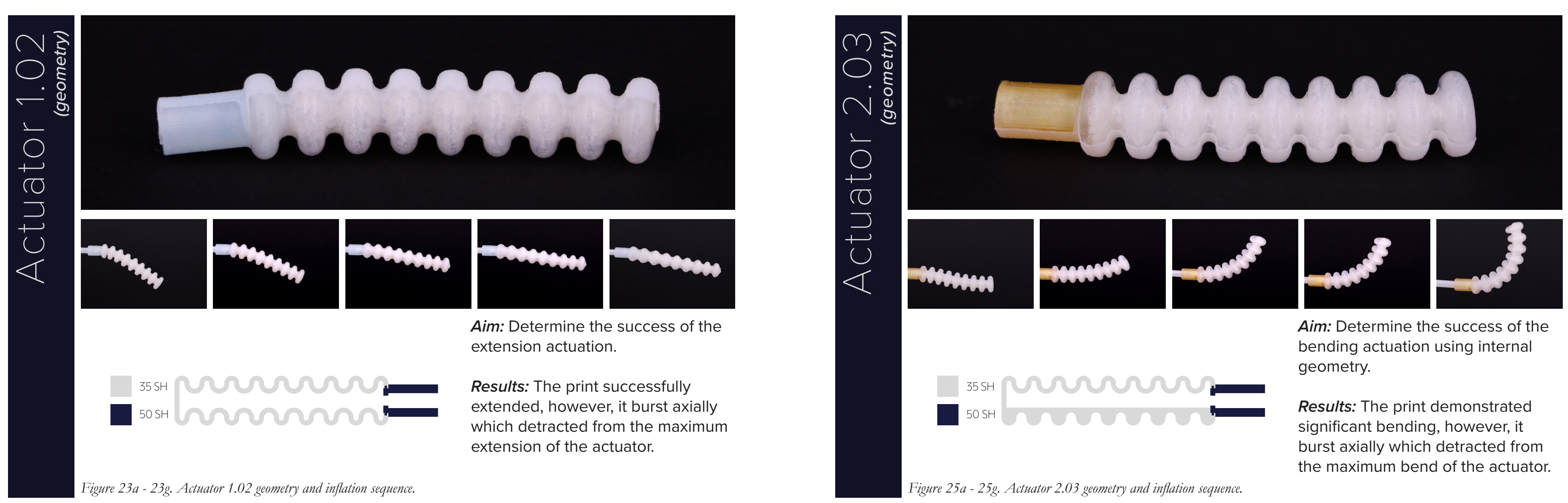

Figure 23a-23g. Actuator 1.02 geometry and inflation sequence.
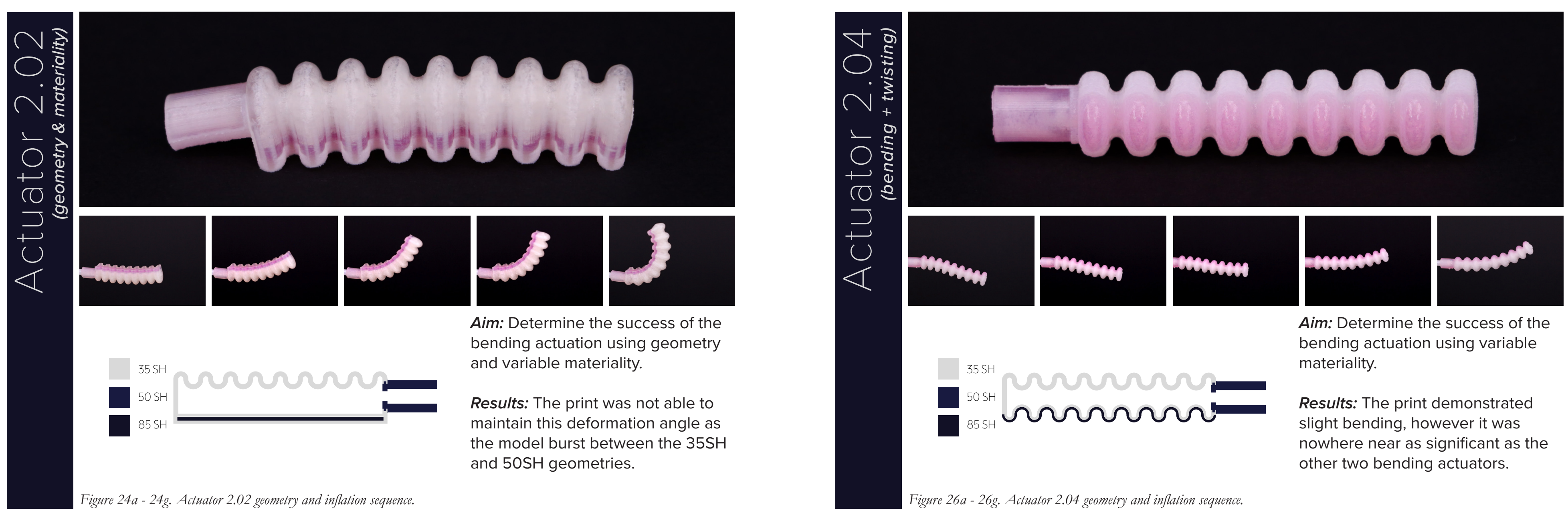


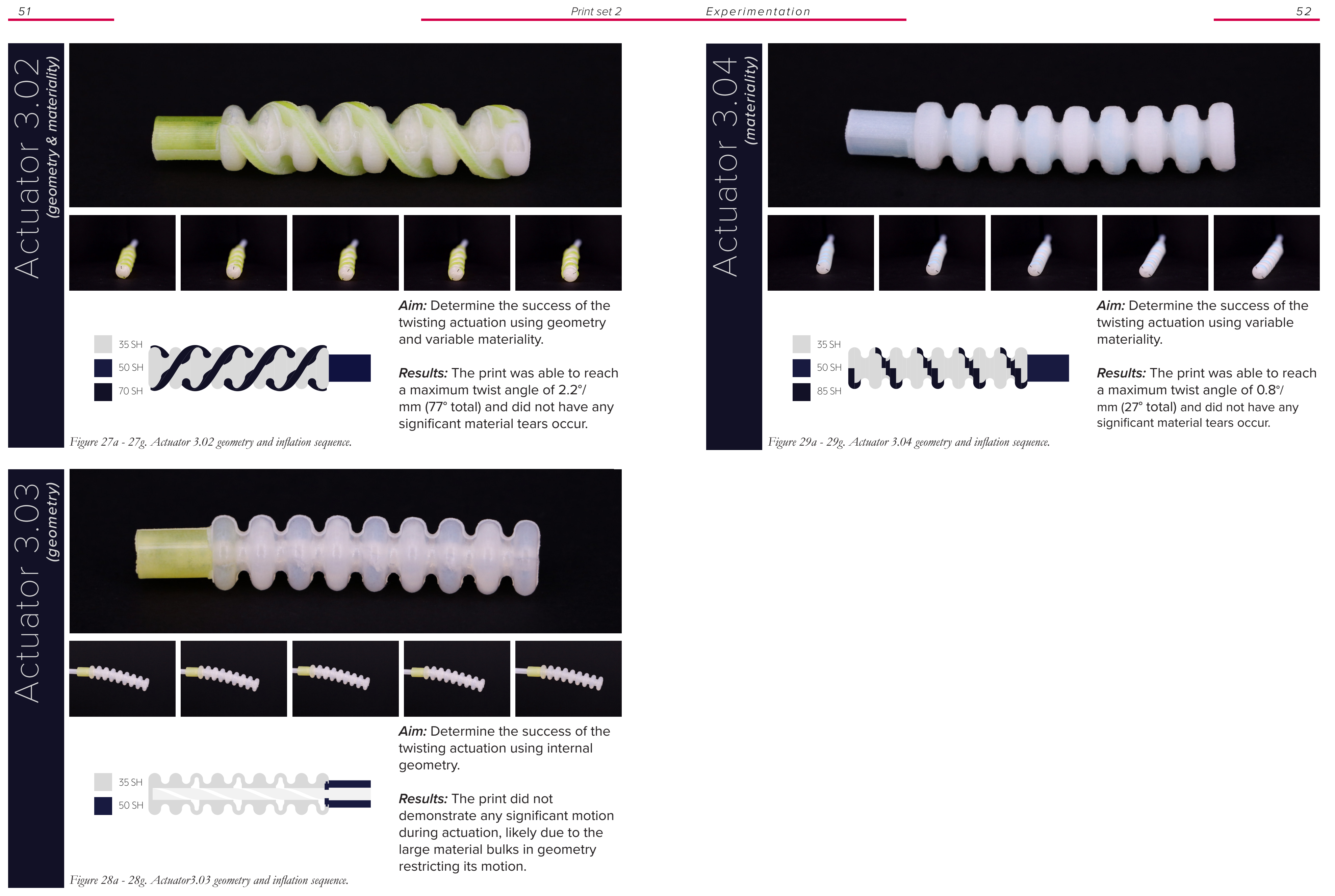




\section{Analysis}

Extension actuator:

The proportions of the extension actuator (1.02)

The geometry + materiality twisting print (3.02) was

the most successful, and it reached a twist angle

extension achieved while maintaining the design's of $77^{\circ}$ along its length of $35 \mathrm{~mm}$. Although this

strength acainst bursting during inflation.

print was successful, a new geometrical solution was explored to further expl angles that are possible.

From a visual inspection, the geometry + materiality The valve that was used to remove interna (2.02) and internal geometry (2.03) prints achieved support material was successful, as it allowed a much larger bending deformation than the more thorough removal of support material and it materiality print (2.04). The main problem with the was easily closed off with superglue once this was bending actuators was the air leaks that limited accomplished.

the deformation angle. This was likely happening

because of the radial asymmetrical nature of the

bending deformation which caused the print layers

to separate along the horizontal axis where air is

escaping. This is addressed in subsequent prits

by altering the is addressed in subsequent prints

regions most susceptible to bursting. print was

Twisting actuators:

$$
\text { complished. }
$$




\section{Print set 3}

\section{Goals}

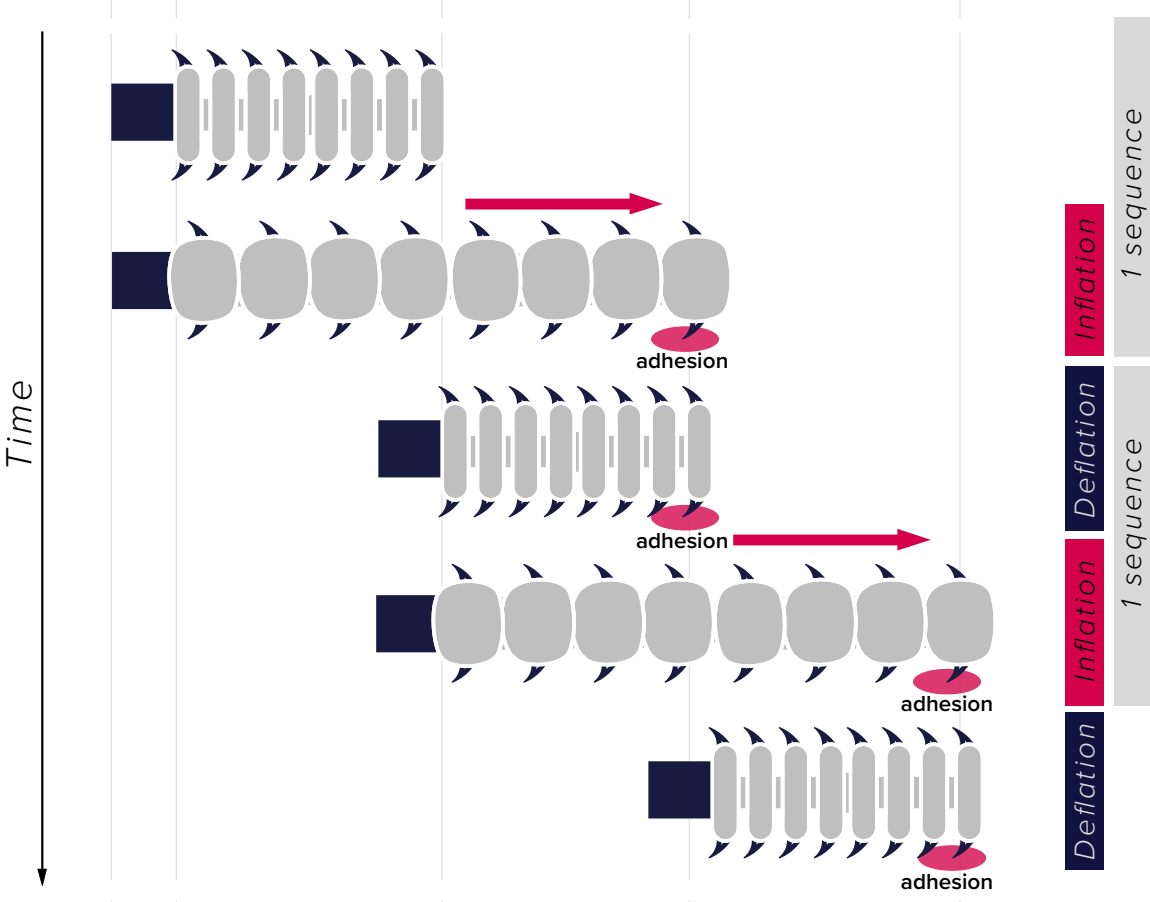

The largest problem with the previous print sets was material tearing. This was addressed in this print set through a set of shore hardness tests which aimed to find the optimal shore hardness in regards to performance and material strength. The other prints in the set were also printed at a large wall thickness (from $0.8 \mathrm{~mm}$ to $1 \mathrm{~mm}$ ) to address this.

In an attempt to increase performance, the bellows on the prints in this set were condensed, meaning that the inner radii were decreased.

The motion that was least satisfactory in its performance for previous prints was the twisting actuator. This was addressed in this print set with a departure from the previous twisting concepts to introduce a completely new geometry. This was tested with different helix angles to see how it would impact perform it would inpact perforn geometry was designed and tested, a precedent in the literature was found which presents a similar geometry for twisting actuation. Development of Multi-chamber Pneumatic Twist Actuator for Soft robot (Darekar et al., 2019) details a method

$3 \mathrm{D}$ printed mould at a much larger scale than the actuators presented in this portfolio. Although the research done by Darekar et al. (2019) is intended for high force applications, the complexity of the fabrication process in comparison to the method presented in this portfolio is notable.

A few other new concepts were tested which were derived from existing concepts:

Extension/adhesion actuator (1.06): This prin was derived from the extension geometry with the addition of adhesion geometry to attempt to achieve linear locomotion (Figure 30).

Expansion actuator (4.01): Derived from the new twisting geometry with the helix angle set to $0^{\circ}$.The concept was designed to achieve a radial expansion upon inflation.

Bending/twisting actuator (2.06): Derived from the bending geometry+materiality model with the bellows offset on an angle from the central axis. This print was designed to follow a helical path upon inflation. 


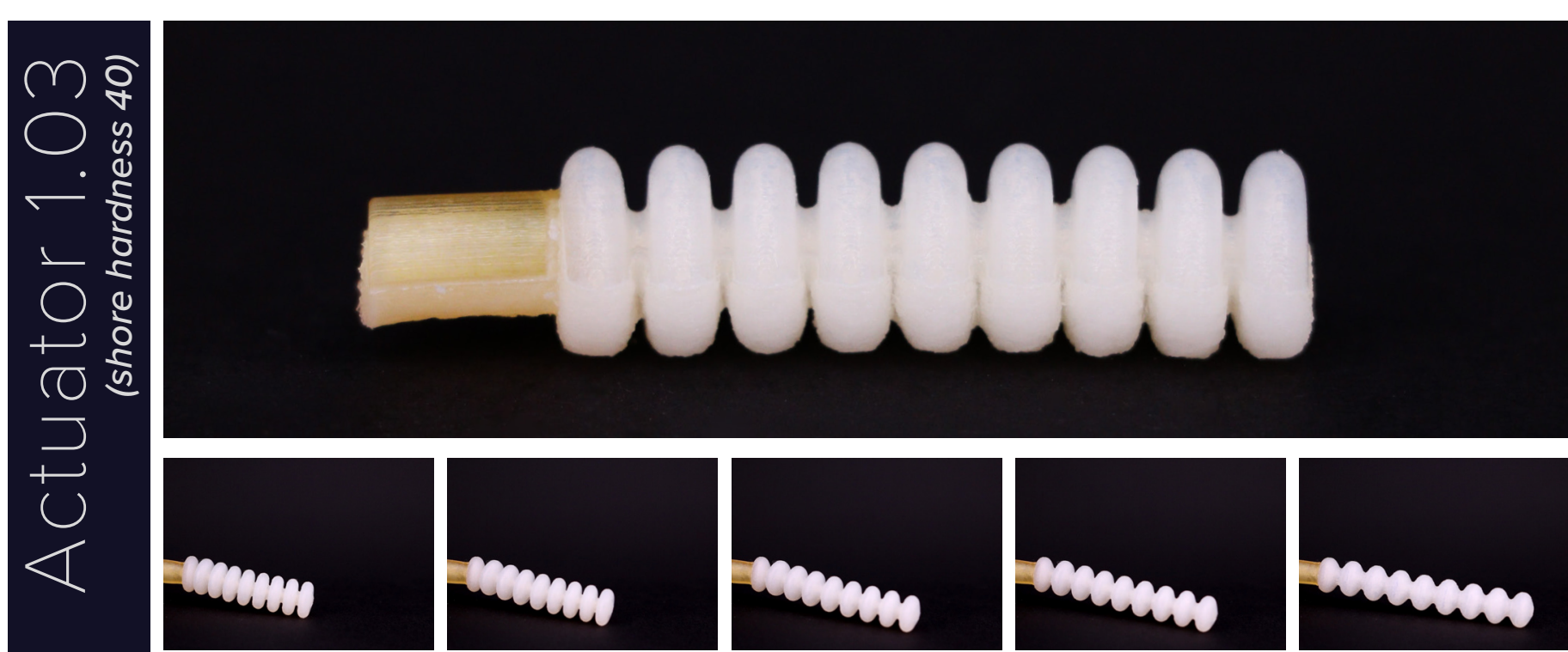

Aim: Determine the best shore hardness for the prints in terms of

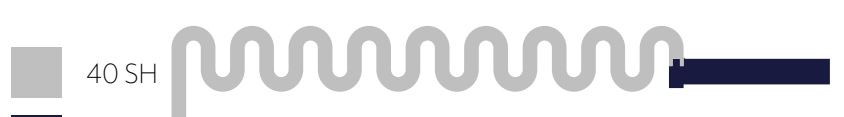

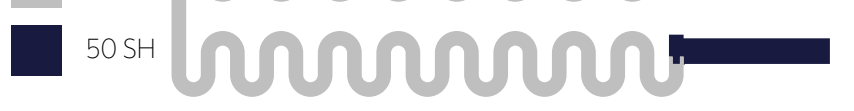
performance and material streng

Results: No material tearing occurred, and the print achieved an extension percentage of $162 \%$.

igure 31a - 31g. Actuator 1.03 geometry and inflation sequence.

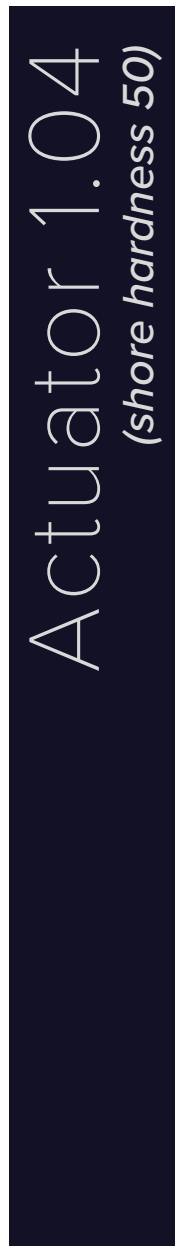

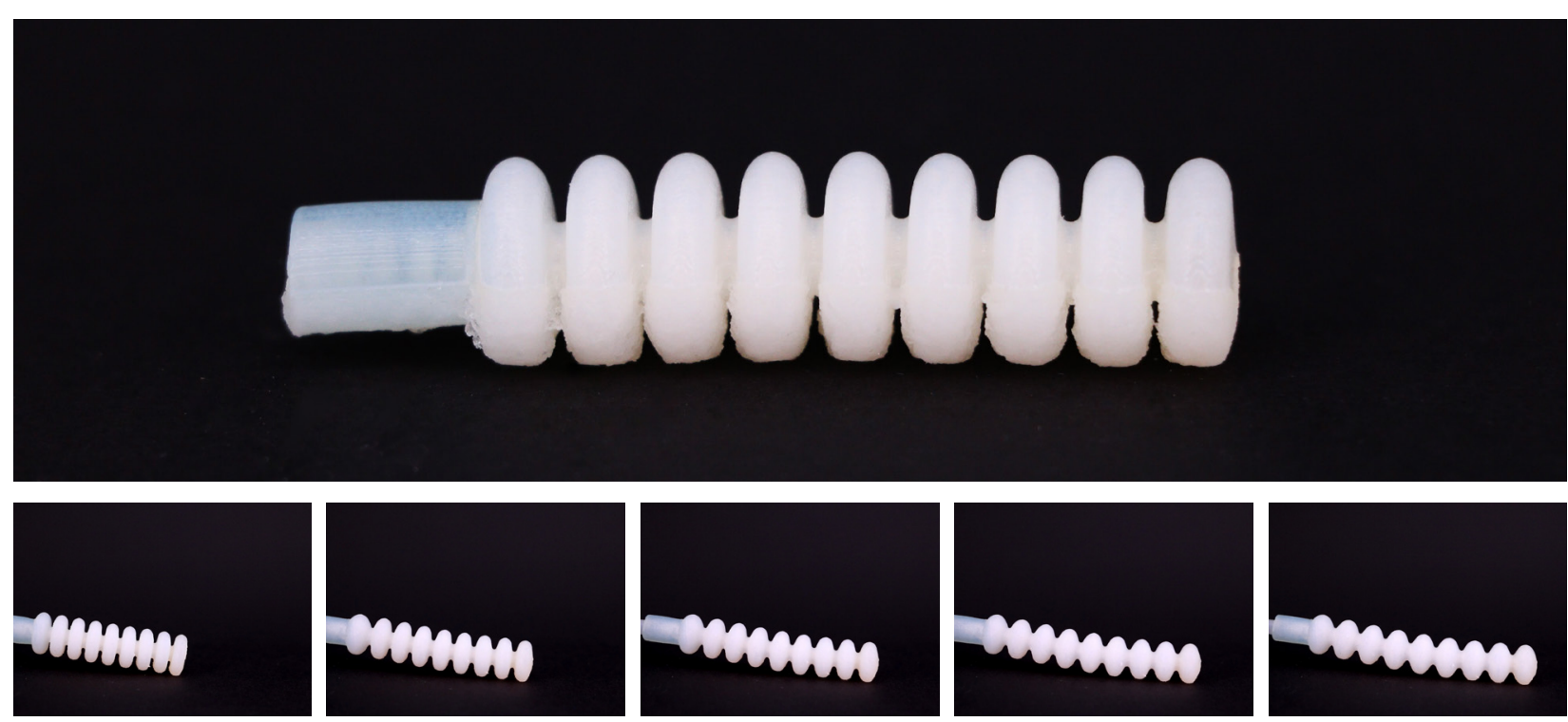

Aim: Determine the best shore hardness for the prints in terms of - crrrrrrrrra performance and material strength.

Results: No material tearing occurred, the print achieved an extension percentage of $153 \%$.

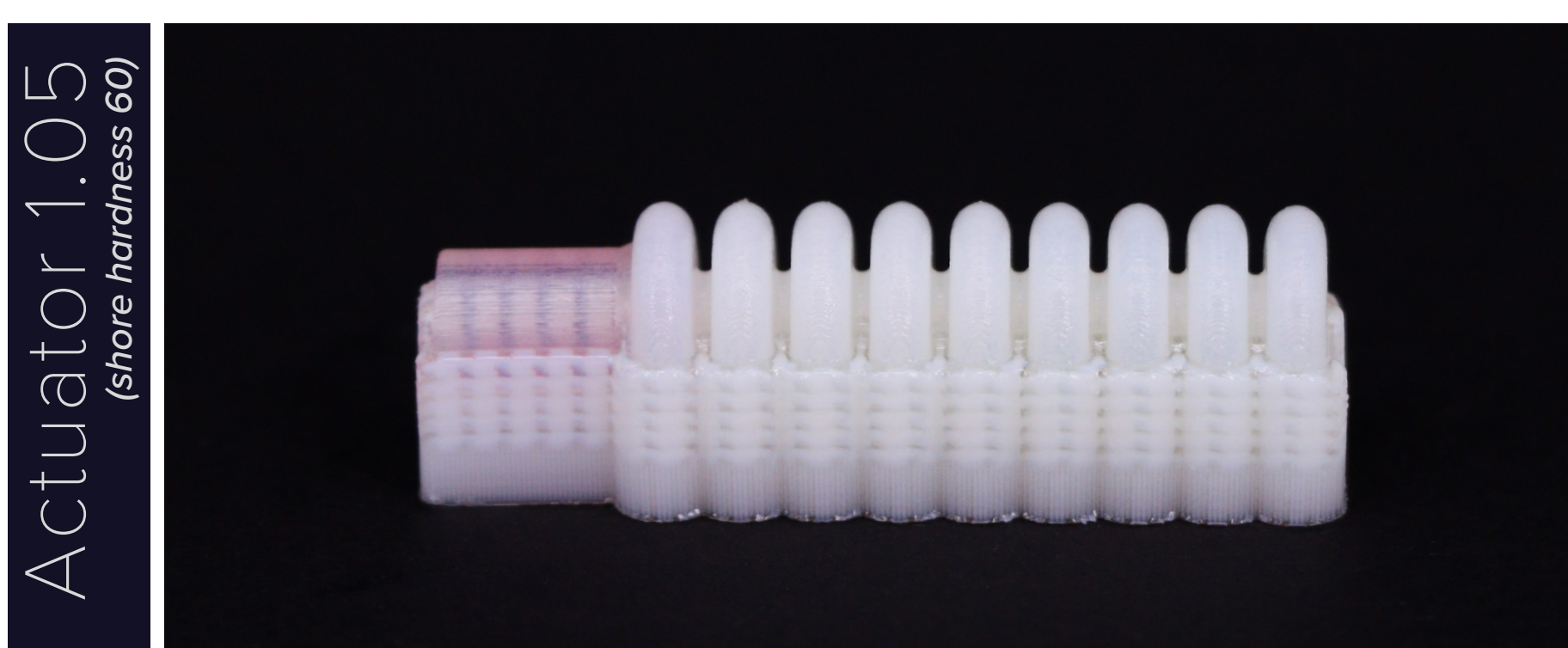

Aim: Determine the best shore hardness for the prints in terms of ${ }_{5 \circ \mathrm{SH}} 0200 \mathrm{n}$ performance and material strength.

Results: Significant material tearing occurred during the cleaning of this print which ruled the print out before inflation testing could occur.
Figure 33a - 33g. Actuator 1.05 geometry and inflation sequence.
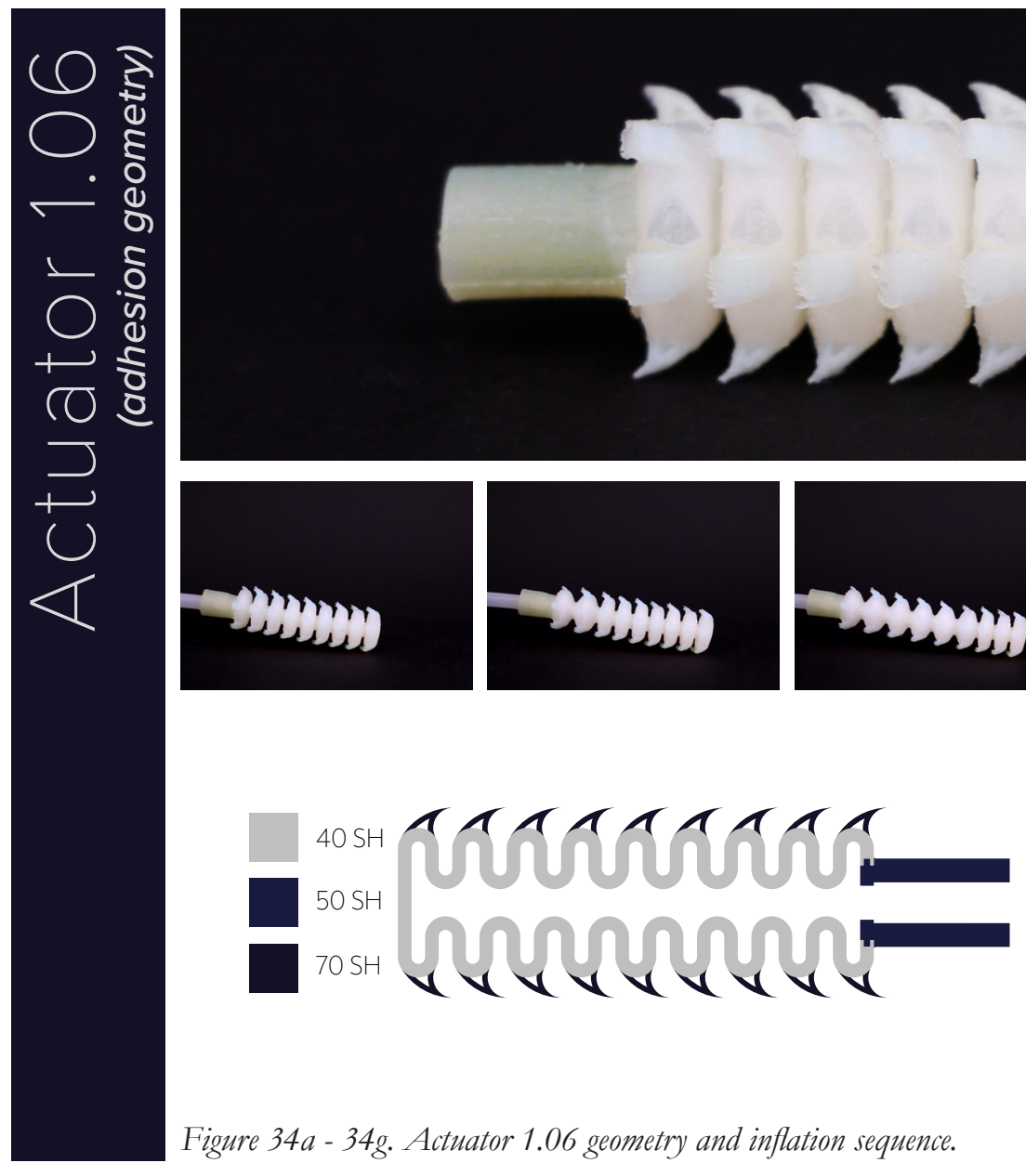

Aim: Create a soft robot capable

of linear locomotion by utilising

knowledge of adhesion.

Results: The extension percentage $157 \%$ was comparable to the other extension geometries, however, the adhesive geometry didn't allow for locomotion to occur. 

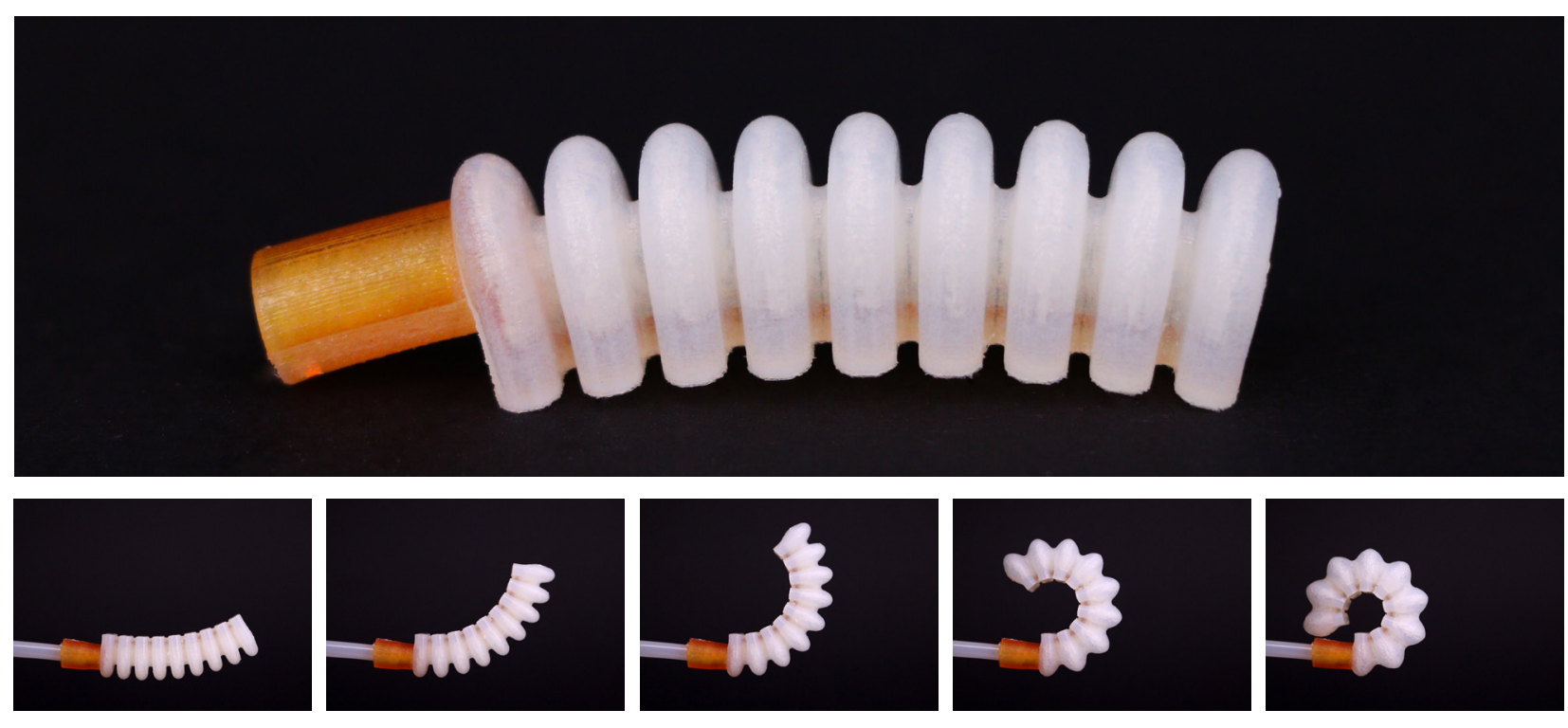

Aim: Determine the success of the

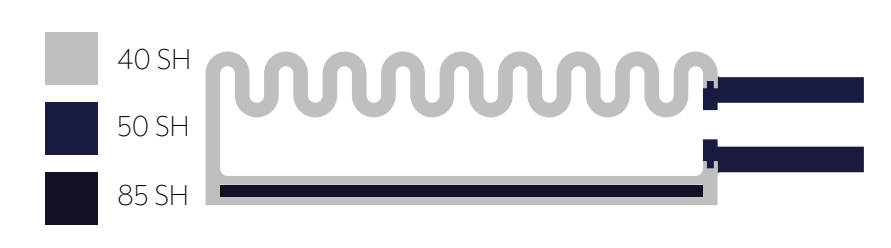

bending actuation using geometry and variable materiality.

Results: No material tearing

occurred, and at its actuated state the print achieved a change in

Figure 35a-35g. Actuator 2.04 geometry and inflation sequence. angle of $7.2^{\circ} / \mathrm{mm}\left(253^{\circ}\right.$ total).
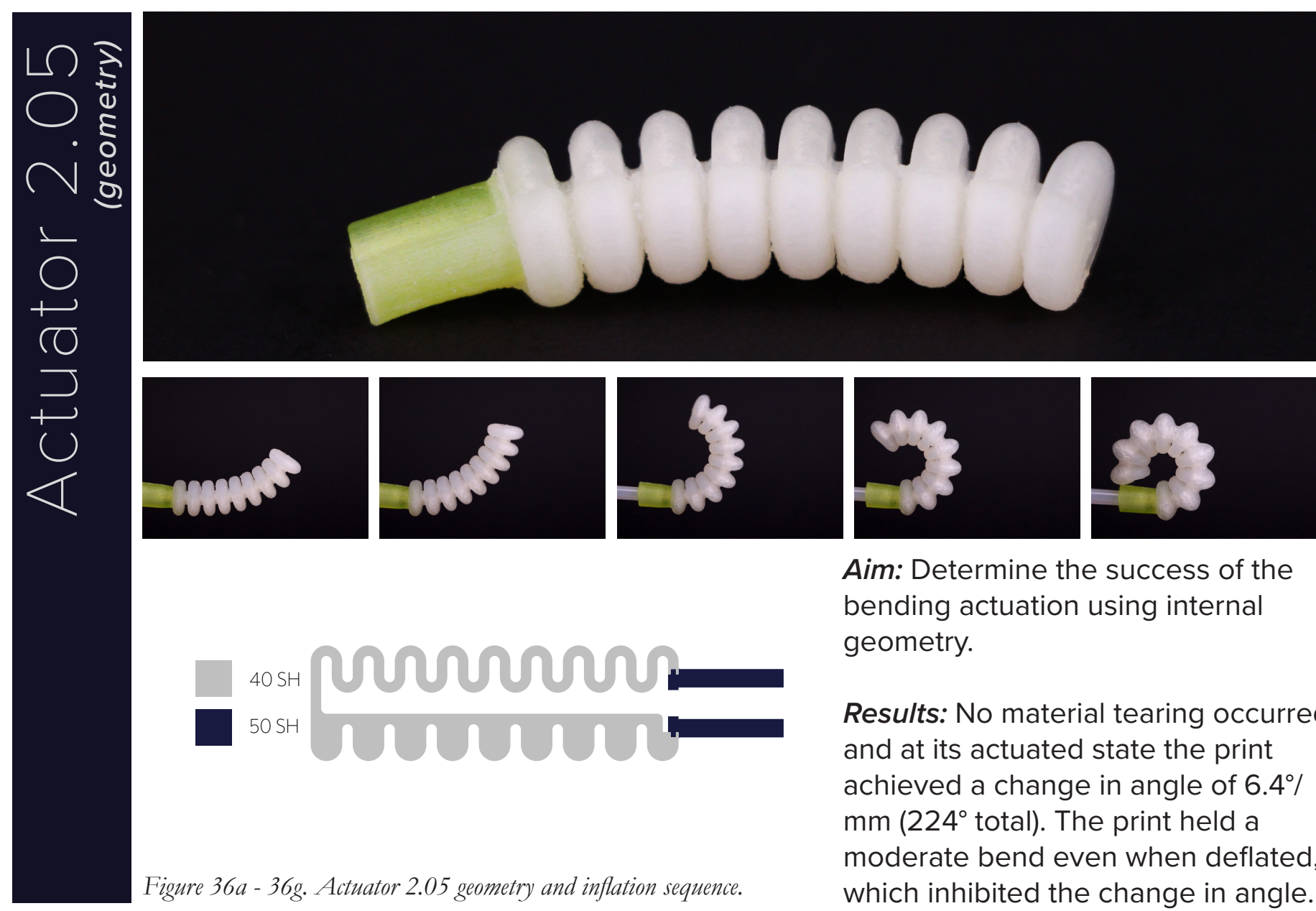

Aim: Determine the success of the bending actuation using internal प geometry.

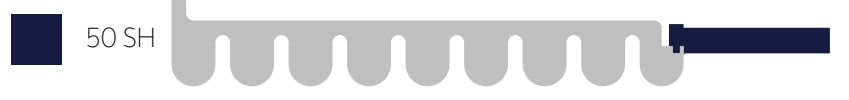

Results: No material tearing occurred, and at its actuated state the print achieved a change in angle of $6.4 \%$ $\mathrm{mm}\left(224^{\circ}\right.$ total). The print held a moderate bend even when deflated, igure 36a -36g. Actuator 2.05 geometry and inflation sequence. which inhibited the change in angle.

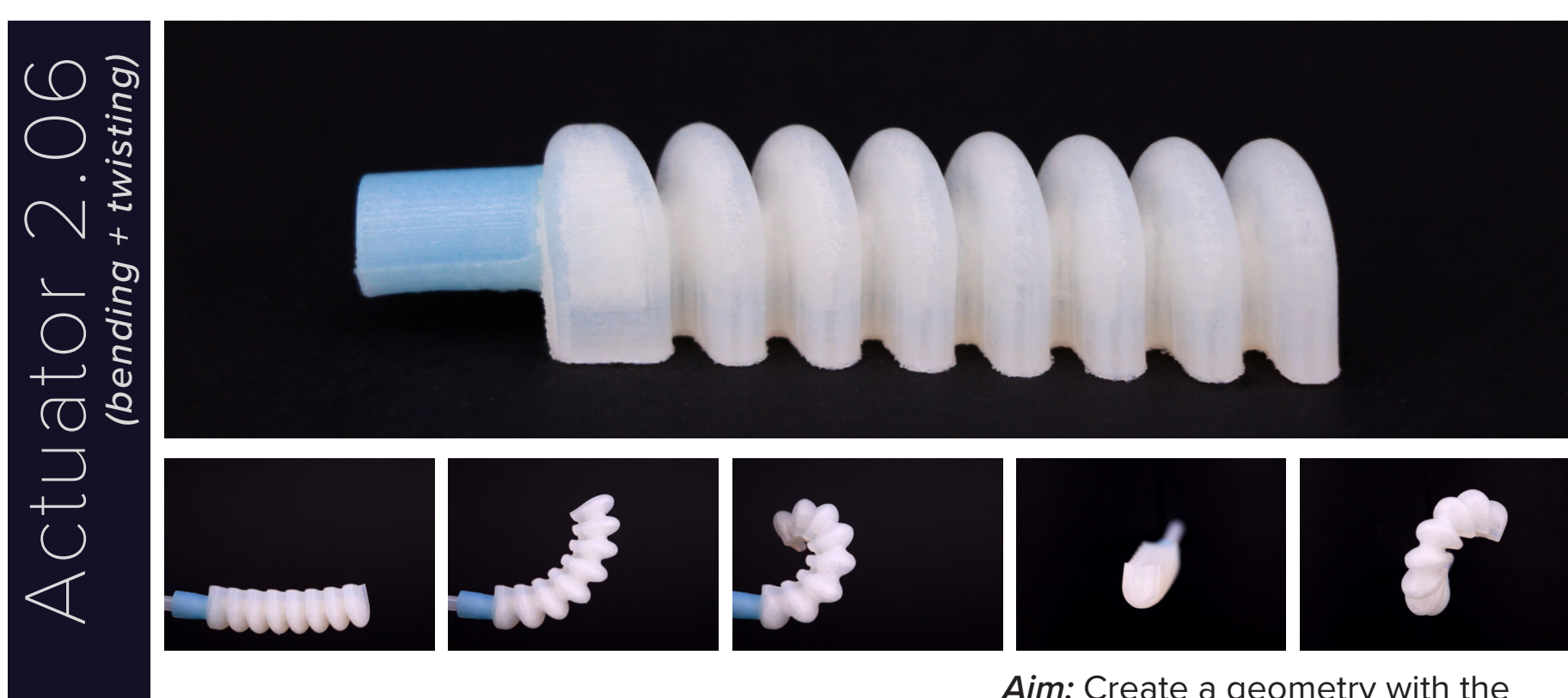

Aim: Create a geometry with the $25^{\circ} \quad$ inflation.

- ${ }^{40 \text { SH }}$ fllow a helical path upon

print successfully followed a helical path.

Figure $37 a-37 g$. Actuator 2.06 geometry and inflation sequence.
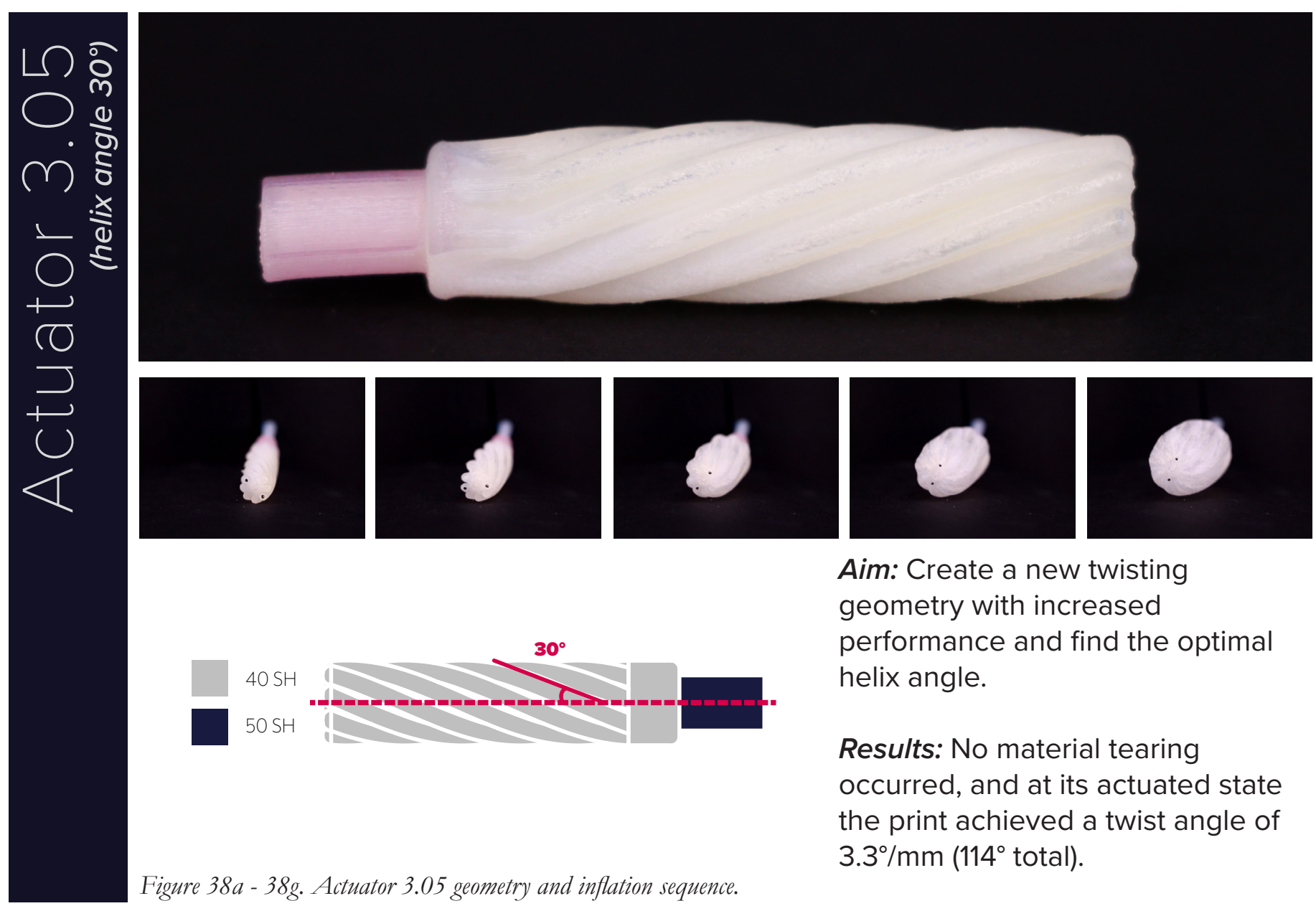

Aim: Create a new twisting geometry with increased

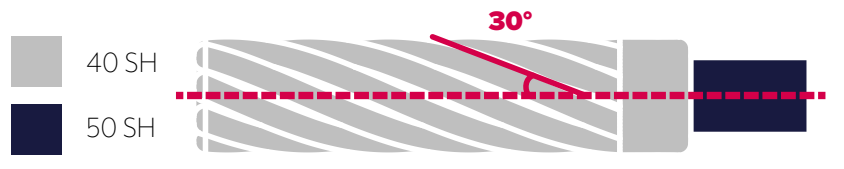

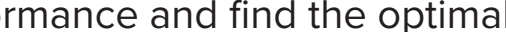

helix angle.

Results: No material tearing occurred, and at its actuated state the print achieved a twist angle of $3.3^{\circ} / \mathrm{mm}\left(114^{\circ}\right.$ total). 


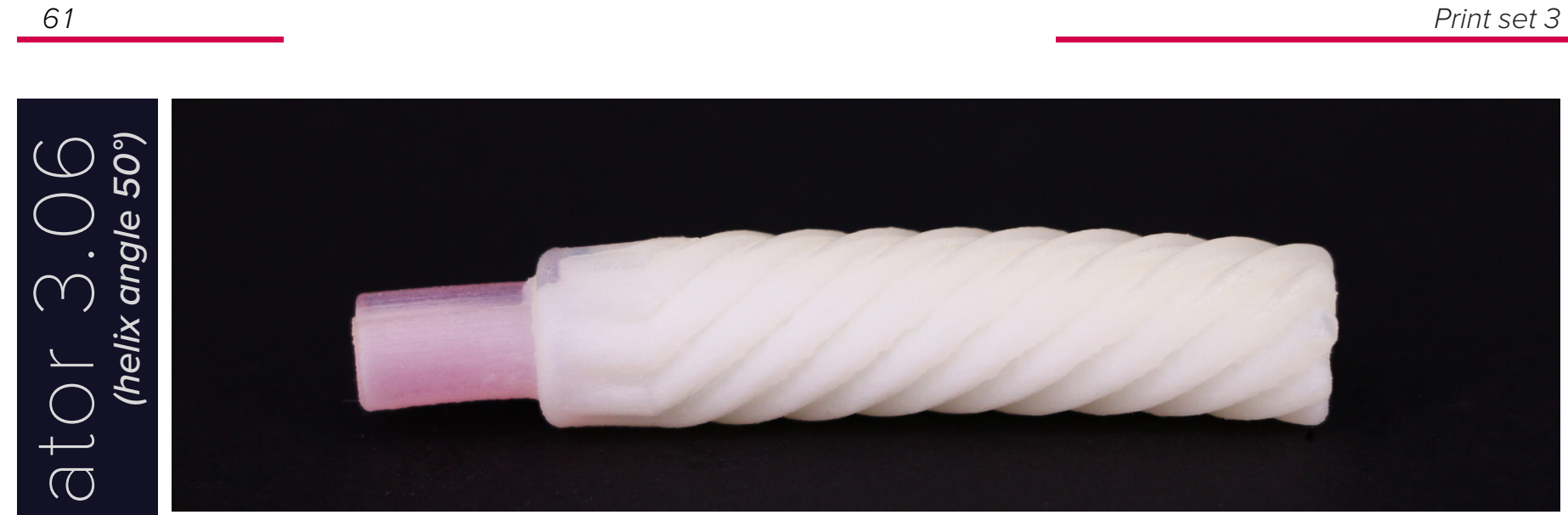

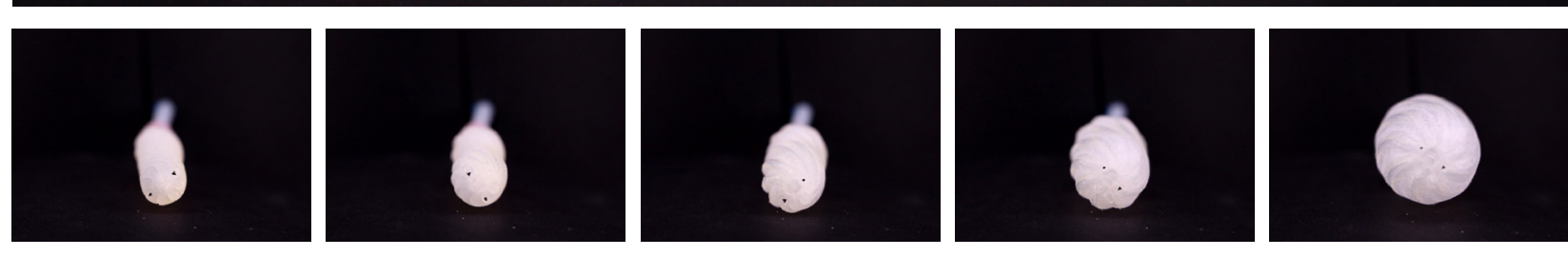

Aim: Create a new twisting geometry with increased

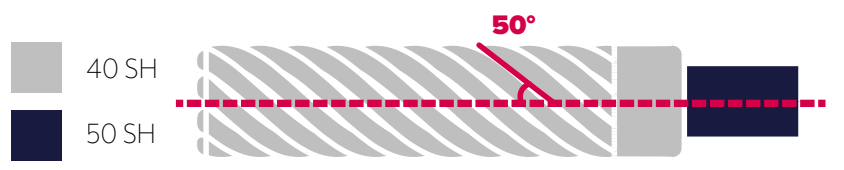

ance and find the optimal

helix angle.

Results: No material tearing occurred, and at its actuated state the print achieved a twist angle of

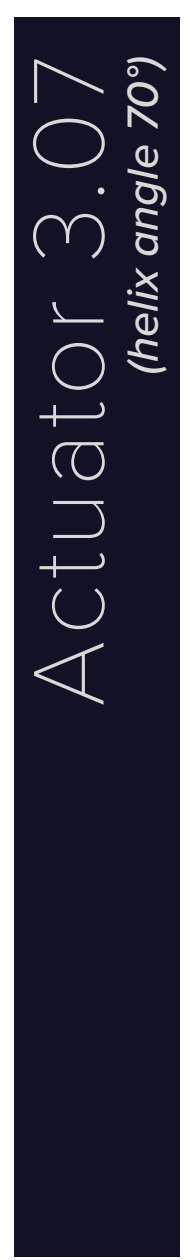

igure 39a - 39g. Actuator 3.06 geometry and inflation sequence.

$7.9^{\circ} / \mathrm{mm}$ ( $277^{\circ}$ total).
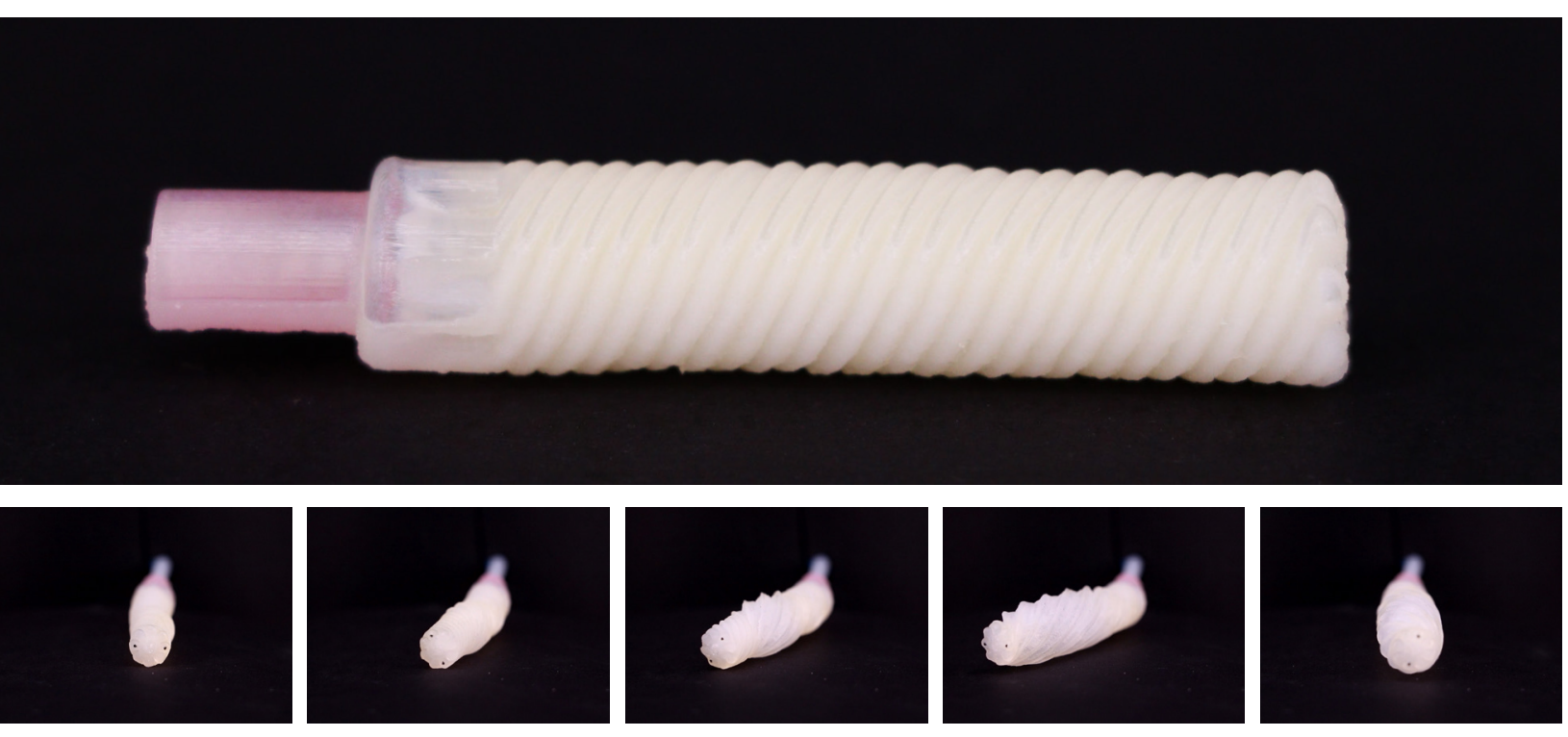

Aim: Create a new twisting

geometry with increased

performance and find the optimal

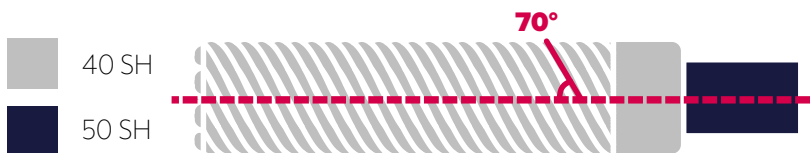

ix angle.

Results: Significant material tearing

occurred, however a twist angle

of $12.4^{\circ} / \mathrm{mm}\left(435^{\circ}\right.$ total) was still

achieved.
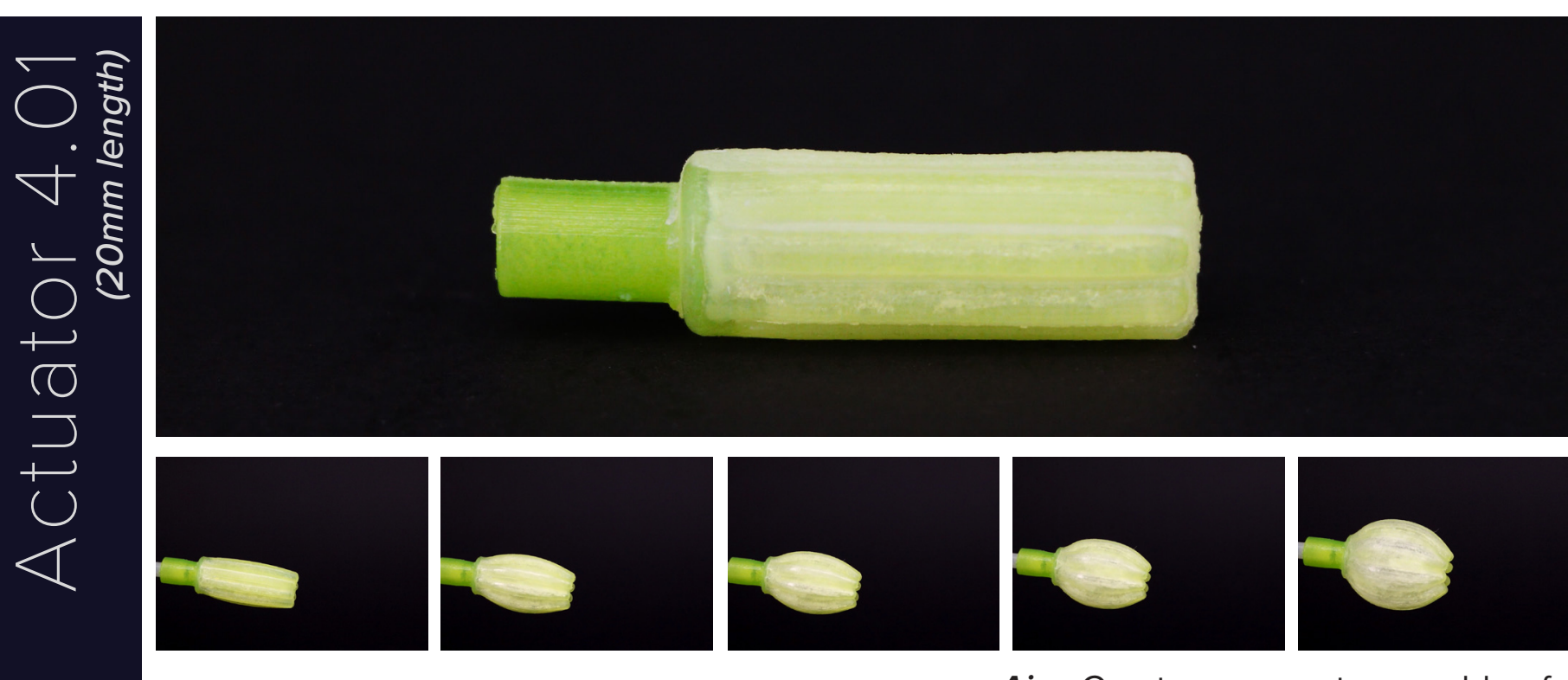

Aim: Create a geometry capable of

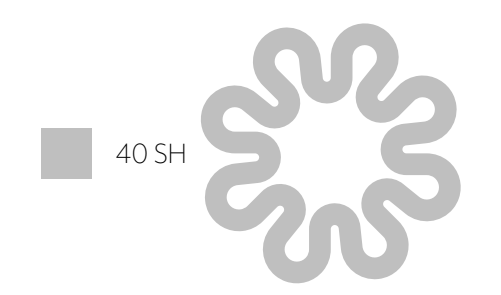

radially expanding upon inflation.

Results: The print successfully

expanded, and reached an

expansion percentage of $182 \%$.

The print burst, and was unable to perform as well afterwards. 


\section{Analysis}

Shore hardness testing:

be because the force pulling the actuator back to its neutral state is larger than the force securing

Shore hardness 40, 50, and 60 were tested as 35 shore hardness was not exhibiting the necessary strength. The $60 \mathrm{SH}$ print (1.05) tore during cleaning deeming it unsuccessful. The $40 \mathrm{SH}$ model (1.03) deeming it unsuccessful. The $40 \mathrm{SH}$ model (1.03)
appeared to give the best result as, although the appeared to give the best result as, although the
$50 \mathrm{SH}$ model (1.04) didn't tear, it exhibited a $9 \%$ drop in performance which was most likely because of the material being stiffer.

\section{Extension-adhesion actuator (1.06):}

The extension-adhesion geometry successfully exhibited extension but not adhesion. The exhesion geometry proved to be very delicate, The many broke off during cleaning and handling. extension percentage of $157 \%$ was comparable to the other extension geometries, meaning the to the other extension geometries, meaning the
adhesion geometry did not have a significant adhesion geometry did not have a significant impact on the extension of the actuator. The actuator was placed on during inflation. This could

\section{to the surface.}

\section{Bending actuators:} materiality focused model (2.04), and an interna geometry modelled with a material bulk on one side of the central axis (2.05). Both tests were successful, each exhibiting over $220^{\circ}$ of total bend. The main difference between the two models was that the internal geometry model did not return to a straight position, and when deflated it always had a significant bend in it ( $\left.60^{\circ}\right)$. This could be the performance if an application required the actuator to return to a straight state. The bending + twisting model (2.06) exhibited the desired actuated state offollowing a helix, however, this was beried state of following a hellx, however, a tighter helix.
The primary bending tests were a geometry
Twisting actuators:

Helix angles of $30^{\circ}, 50^{\circ}$, and $70^{\circ}$ were printed and tested with the same length $(35 \mathrm{~mm})$ and base geometry sketch. The $70^{\circ}$ model (3.07) twisted the most along its length at $435^{\circ}\left(12.4^{\circ} / \mathrm{mm}\right)$ however it also had issues inflating fully. Since the geometry was the most tightly wound, some of the geometry fused together during printing which restricted its motion. The geometry was also compared to the material twist model and the materiality + geometry twist model from print set 2 (3.02), which showed that the new geometry significantly outperformed

\section{Expansion actuator (4.01):}

The expansion geometry worked as anticipated and reached an expansion percentage of $182 \%$. It was designed using the same base geometry as the twisting actuators. 


\section{Print set 4}

\section{Goals}

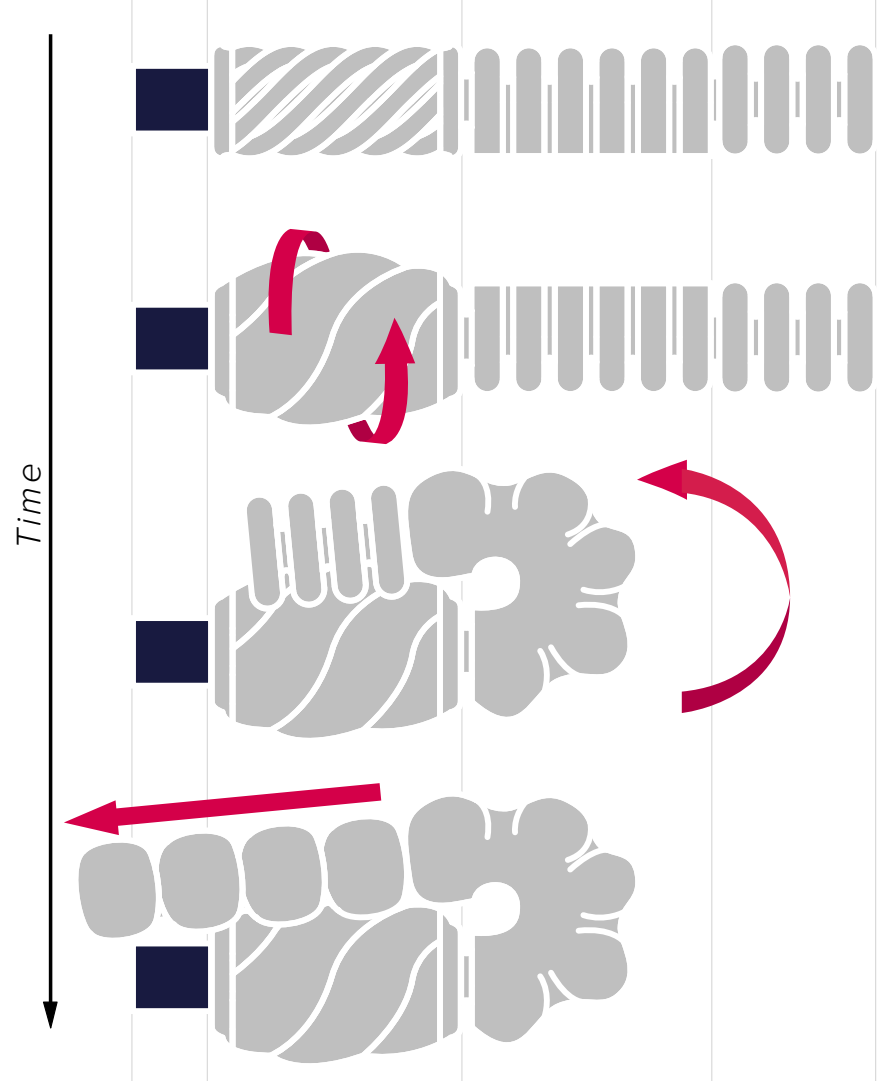

To optimise the optimal helix angle for the twisting A series of internal valves were tested, which, if geometry which maximises both twist angle and successful, would stop the inflation of the second performance, three further iterations of the same half of the actuator until it reaches a certain base geometry were tested. These included helix pressure. The internal valves were placed halfway angles of $55^{\circ}, 60^{\circ}$, and $65^{\circ}$, because the $70^{\circ}$ helix along the bellows of the extension geometry, and angle print from print set 3 had significant tears geometric tolerances of $0.05 \mathrm{~mm}, 0.1 \mathrm{~mm}, 0.15 \mathrm{~mm}$, due to the steepness of the helix angle. Therefore, and $0.2 \mathrm{~mm}$ were printed.

it was hypothesised that the optimal helix angle would be between $50^{\circ}$ and $70^{\circ}$.

The first model with multiple sections of different motion geometries was also printed in this set. The To test how the expansion percentage scales, the goal was to see how successful the actuation would expansion print geometry was altered to have be when multiple geometries were being inflated in a length of $35 \mathrm{~mm}$ rather than $20 \mathrm{~mm}$ in the first unison (Figure 42)

expansion print. This also made it the same length

as the other actuators being tested. 


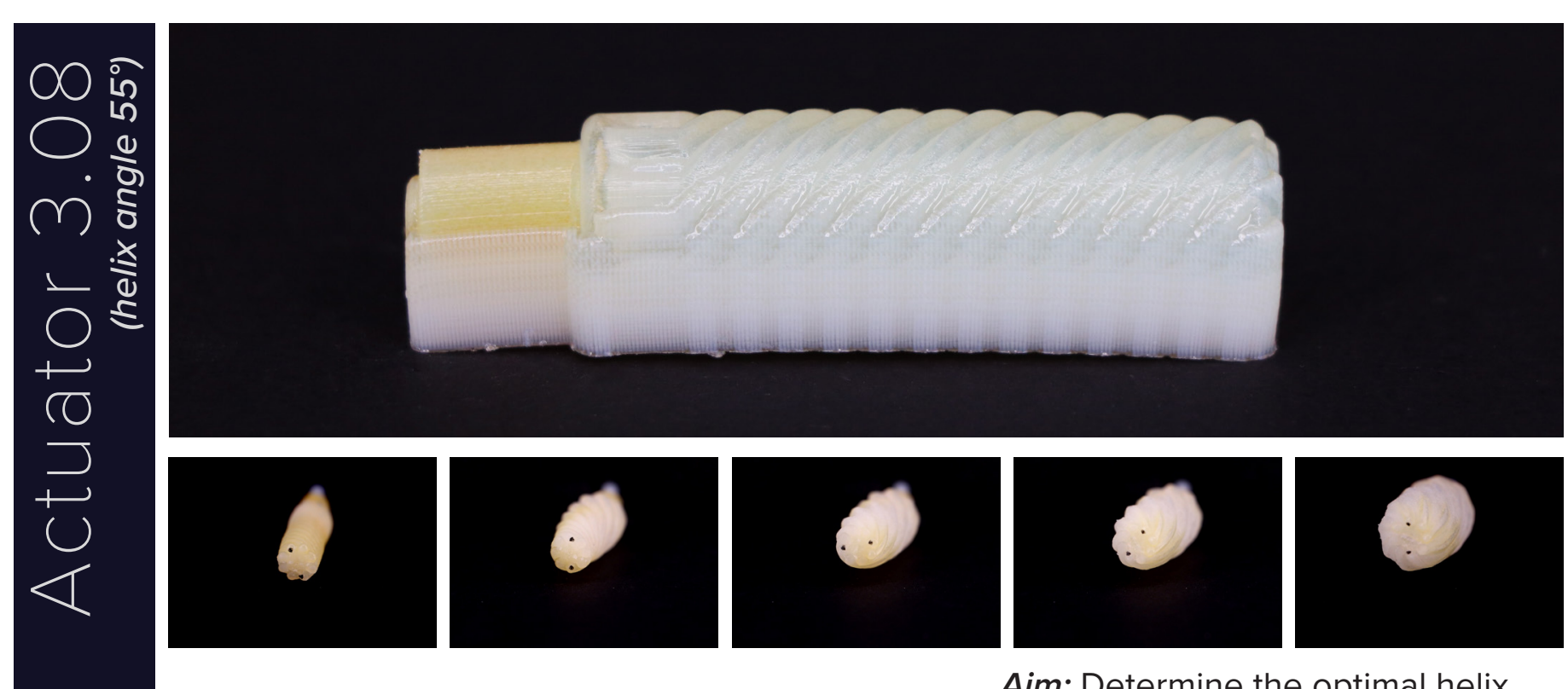

Aim: Determine the optimal helix angle to maximise twist angle whilst

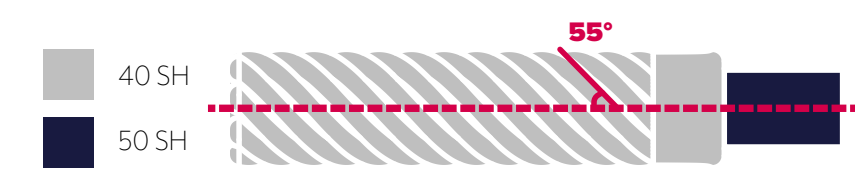

aintaining performance.

Results: No material tearing

occurred, and at its actuated state the print achieved a twist angle of $9.7^{\circ} / \mathrm{mm}$ (339 ${ }^{\circ}$ total).

Figure 43a - 43g. Actuator 3.08 geometry and inflation sequence.

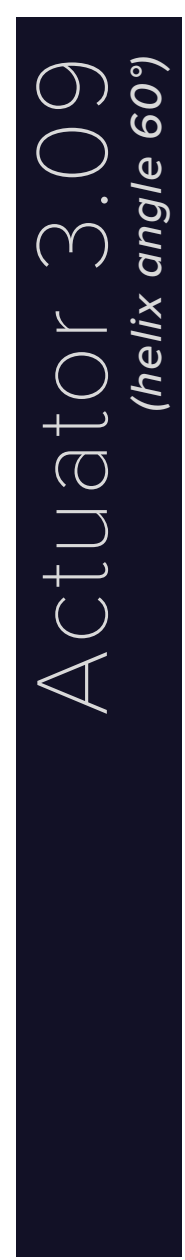

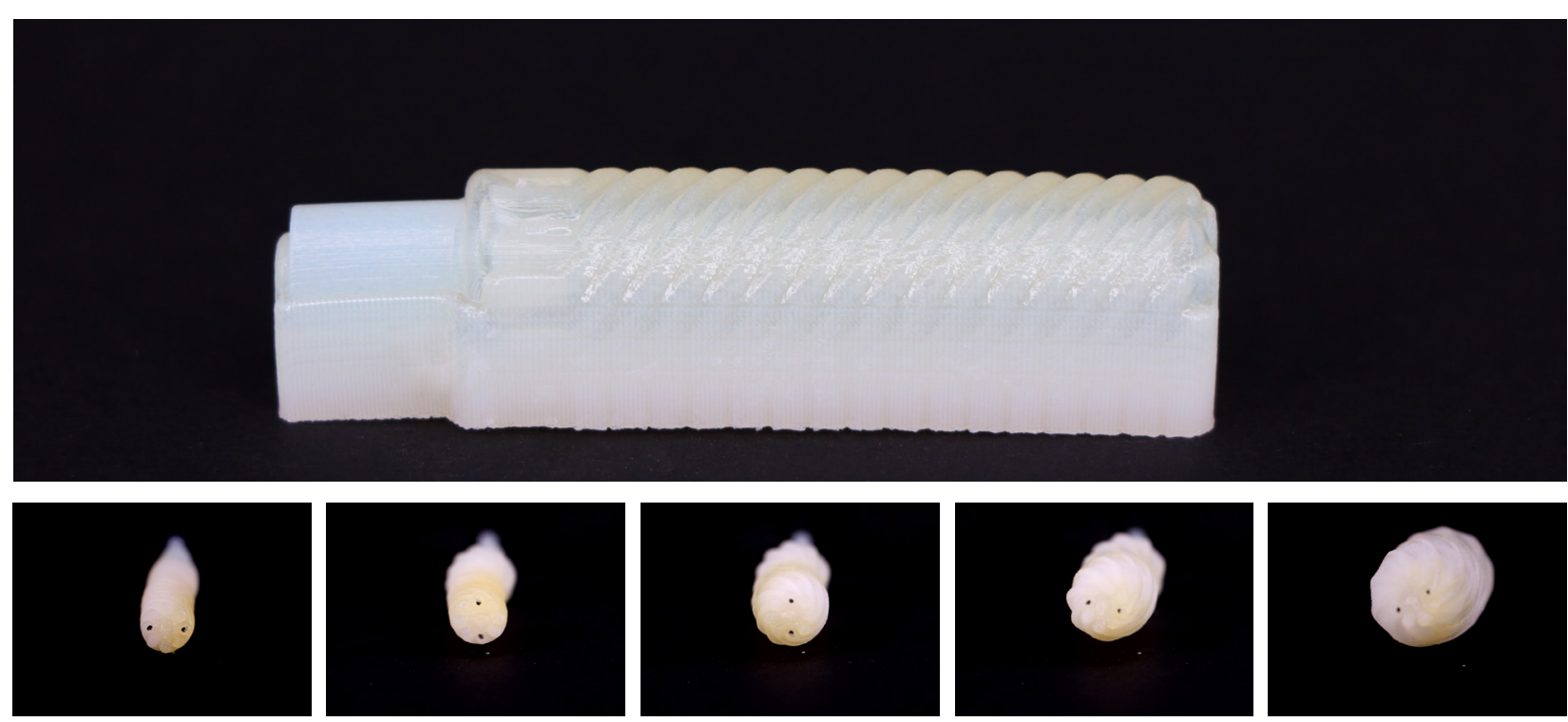

Aim: Determine the optimal helix angle to maximise twist angle whilst maintaining performance.

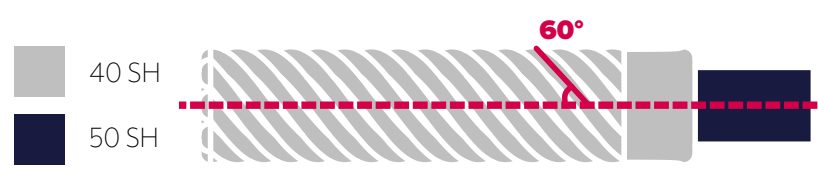

Results: No material tearing

occurred, and at its actuated state the print achieved a twist angle of $11.3^{\circ} / \mathrm{mm}\left(394^{\circ}\right.$ total)
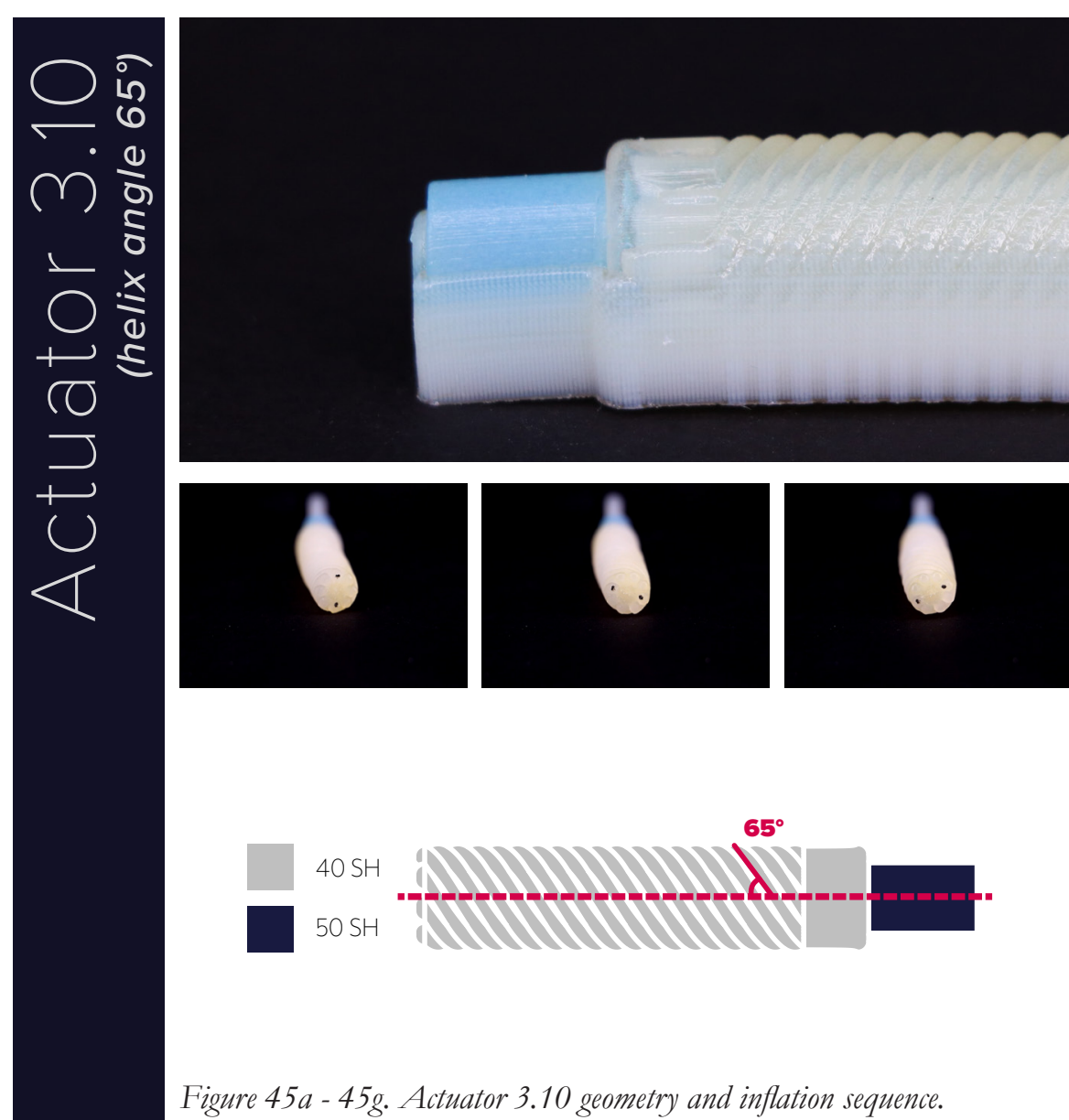

Aim: Determine the optimal helix angle to maximise twist angle whilst maintaining performance.

Results: Significant materia tearing occurred, resulting in a comparatively low twist angle of $6.4^{\circ} \mathrm{mm}$ ( $255^{\circ}$ total) being achieved.

Fure 45a-45g. Actuator 3.10 geometry and inflation sequence.
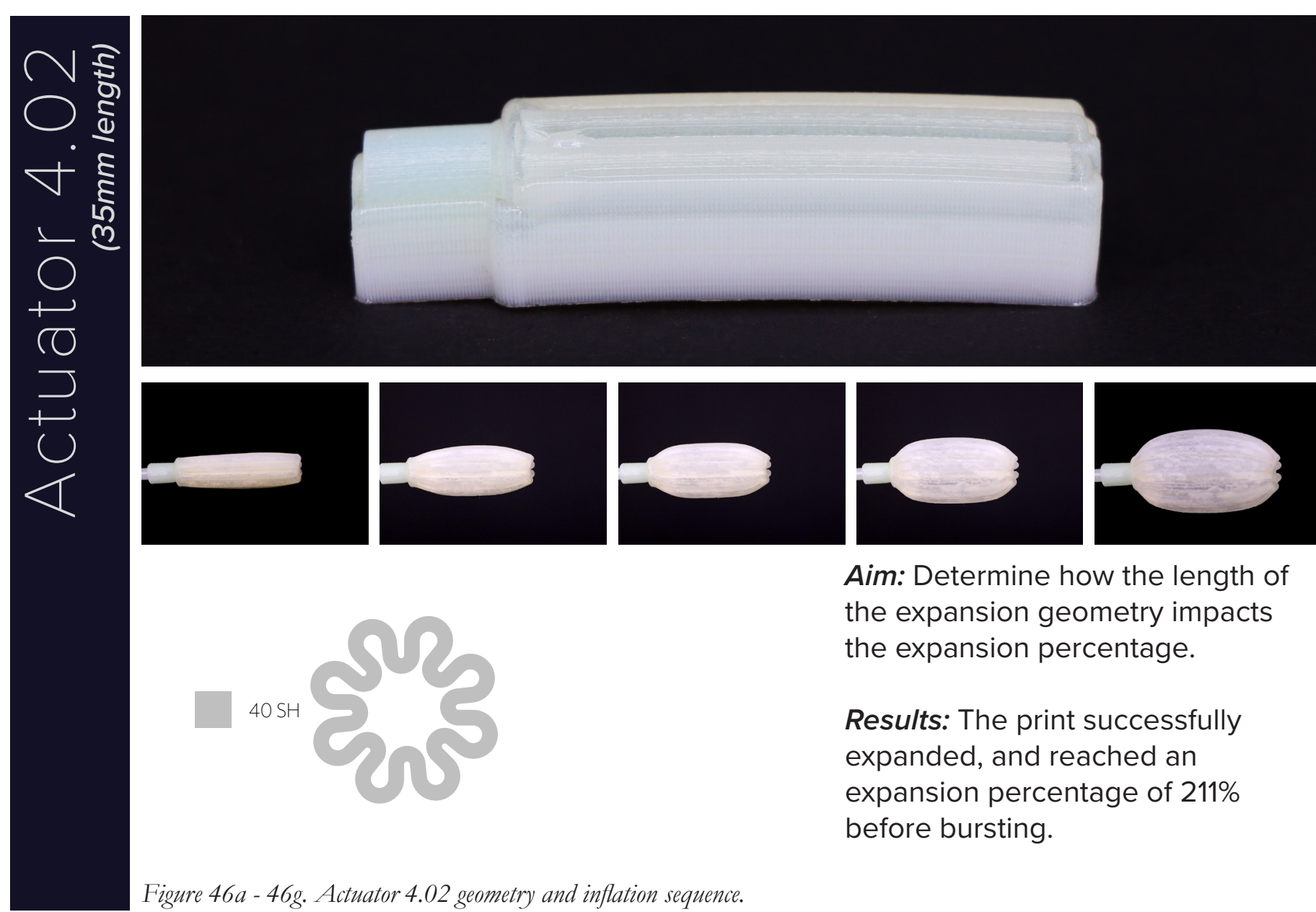

Aim: Determine how the length of ${ }_{40 \mathrm{SH}}$ the expansion geometry impacts the expansion percentage.

Results: The print successfully expanded, and reached an expansion percentage of $211 \%$ before bursting. 


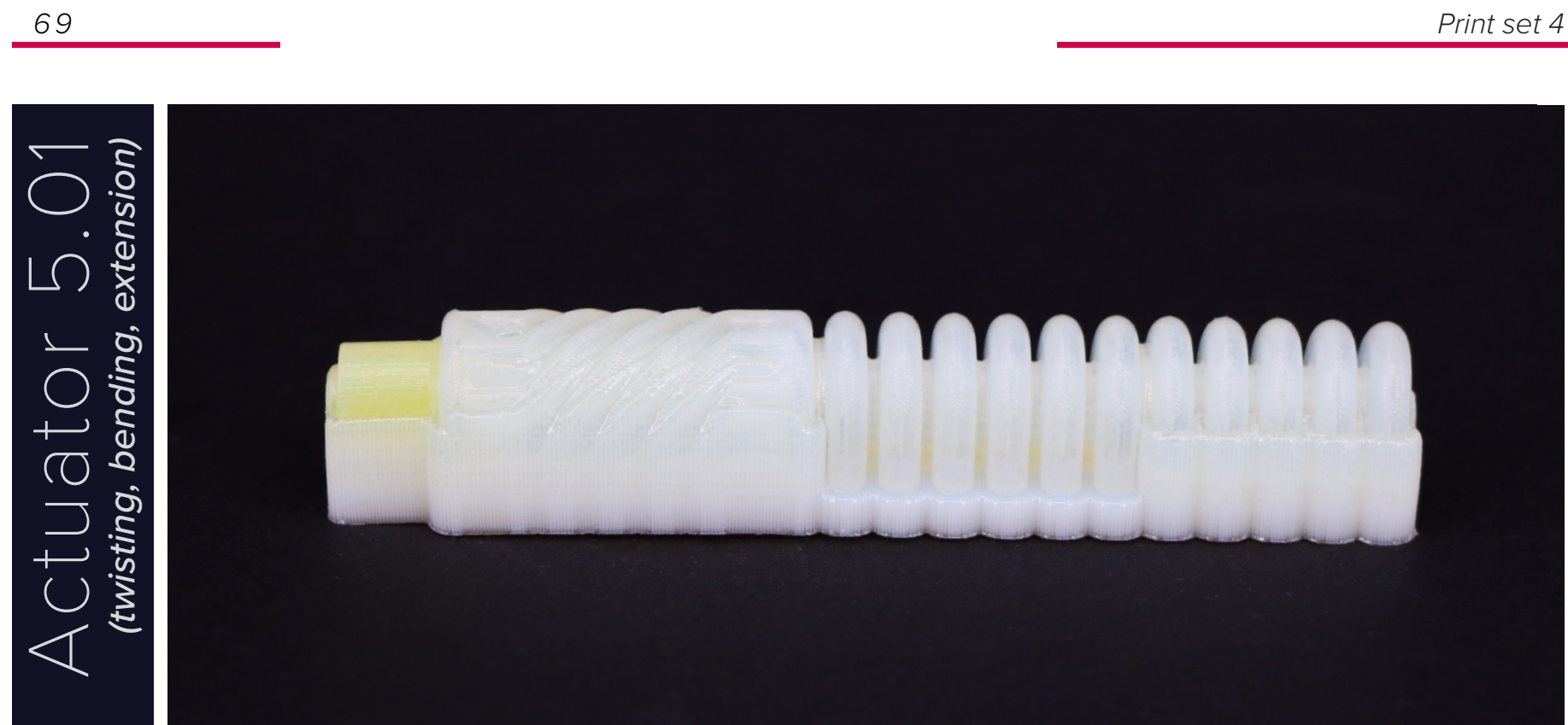

Aim: Create a soft robot capable of achieving multiple motions through one

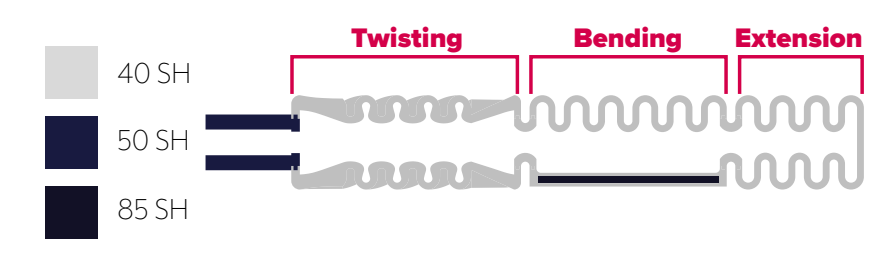

Results: Large tears occurred in the connections between sections. Rough actuation testing showed that the twisting section actuated before the other sections could reach actuation

Figure 47a - 47b. Actuator 5.01 geometry. pressure.

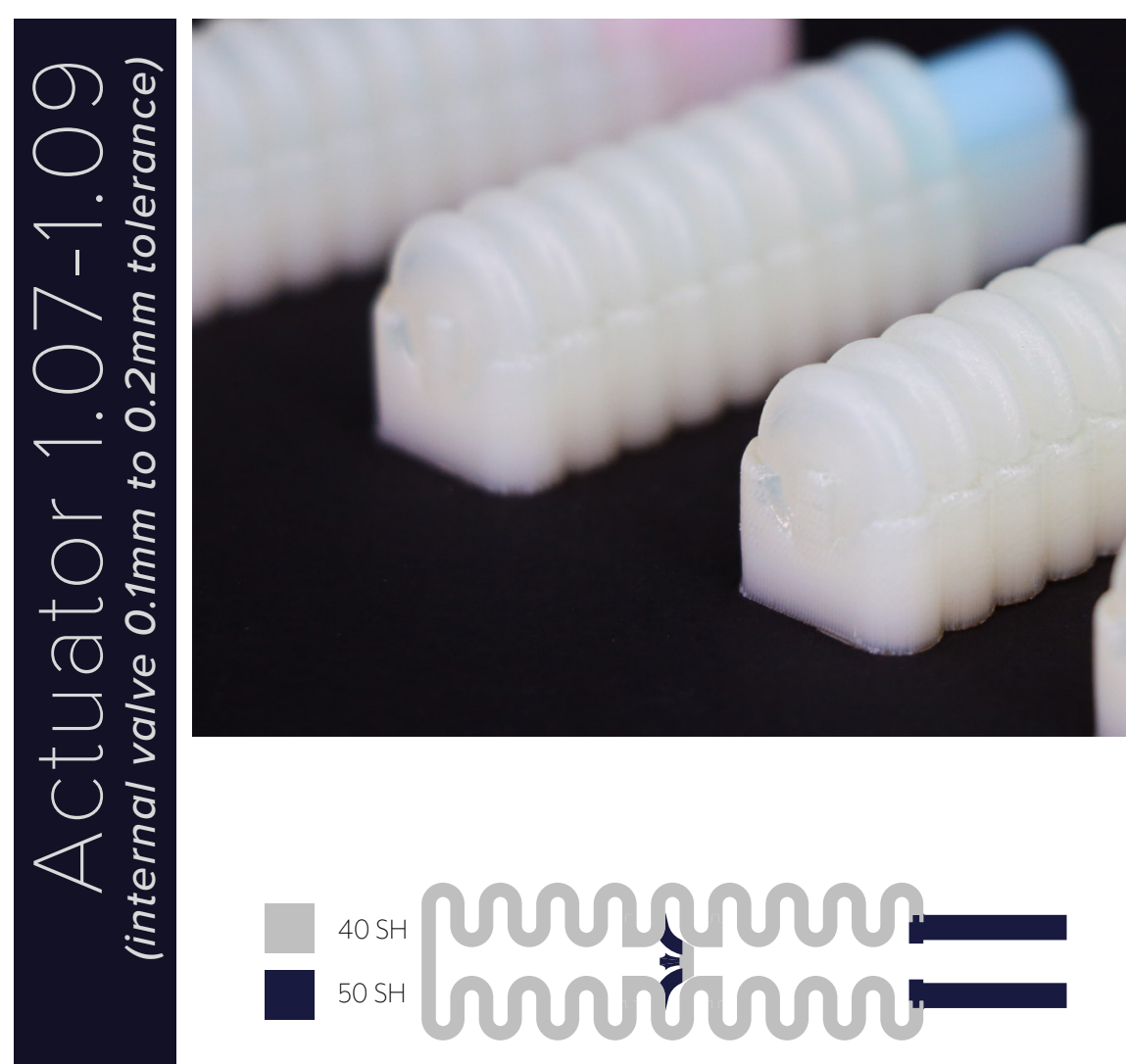

Aim: Create a valve capable of controlling the sequence of

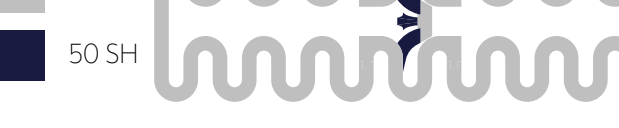

Results: As the $0.05 \mathrm{~mm}$ tolerance valve failed, the larger gaps were assumed to also not restrict the

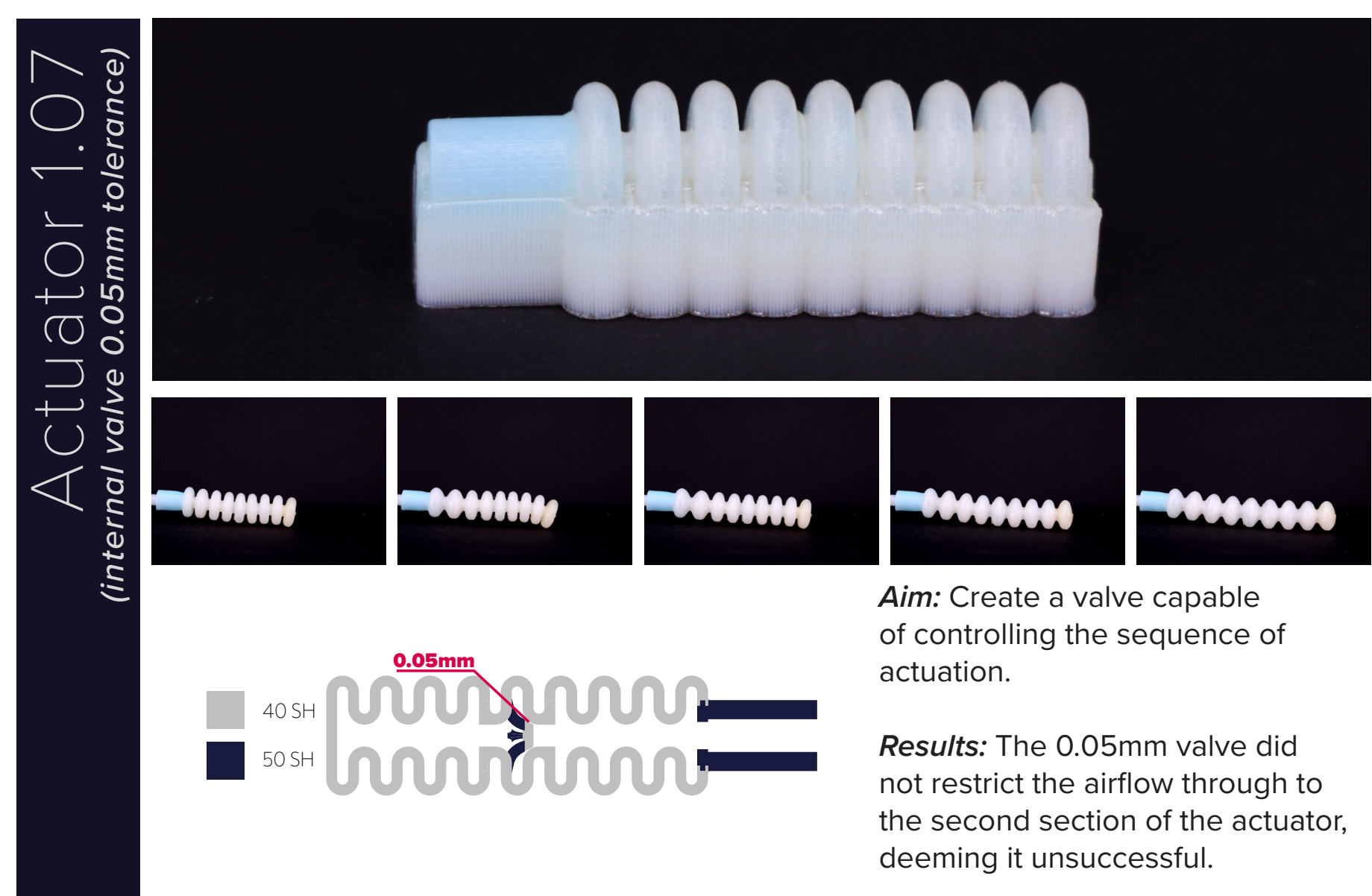




\section{Analysis}

Helix angles of $55^{\circ}, 60^{\circ}$, and $65^{\circ}$ were tested in this This print, although it tore before proper actuation print set. The $65^{\circ}$ helix angle burst before it could testing could be carried out, highlighted a key issue fully inflate, likely due to the steepness of the helix for complex actuation. The actuator was tested angle. The $55^{\circ}$ and $60^{\circ}$ prints worked well, although through rough inflation testing with a ball pump, the $60^{\circ}$ print was irregular in its performance - where the twisting section inflated and burst before sometimes only parts of the geometry would inflate. the bending and extension sections reached a As the performance of the $55^{\circ}$ helix angle was the large enough pressure to inflate. As the twisting most predictable while still reaching a larger twist section has a different base geometry to the other angle than the $50^{\circ}$ print, it was determined to be two sections, the pressure required for inflation the most successful of the twisting prints.

was significantly different.

\section{Expansion actuator (4.02):}

\section{Internal valves:}

The expansion geometry actuated in the same way The internal valve design tested in this print set was as the first expansion print did, however, the longer unsuccessful as it did not cause the first section length $(35 \mathrm{~mm}$ as opposed to $20 \mathrm{~mm}$ ) allowed for to inflate before allowing air into the second a $29 \%$ increase in expansion percentage. This section. This could be due to certain intricacies print also appeared to burst more quickly than the in the geometry or due to allowing for too large shorter version, which could be due to the larger a geometric tolerance. However, it was also very expansion percentage.

likely that the geometry of the valve was too small and delicate, and was either broken during cleaning or was completely ineffective during inflation. 


\section{Print set 5}

\section{Goals}

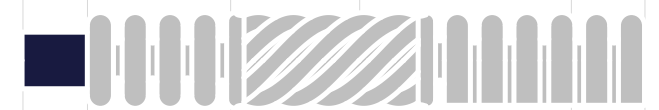 \\ $\overrightarrow{-000}$ romannma \\ - $0000($ / flumu \\ - $0000(2003)$}

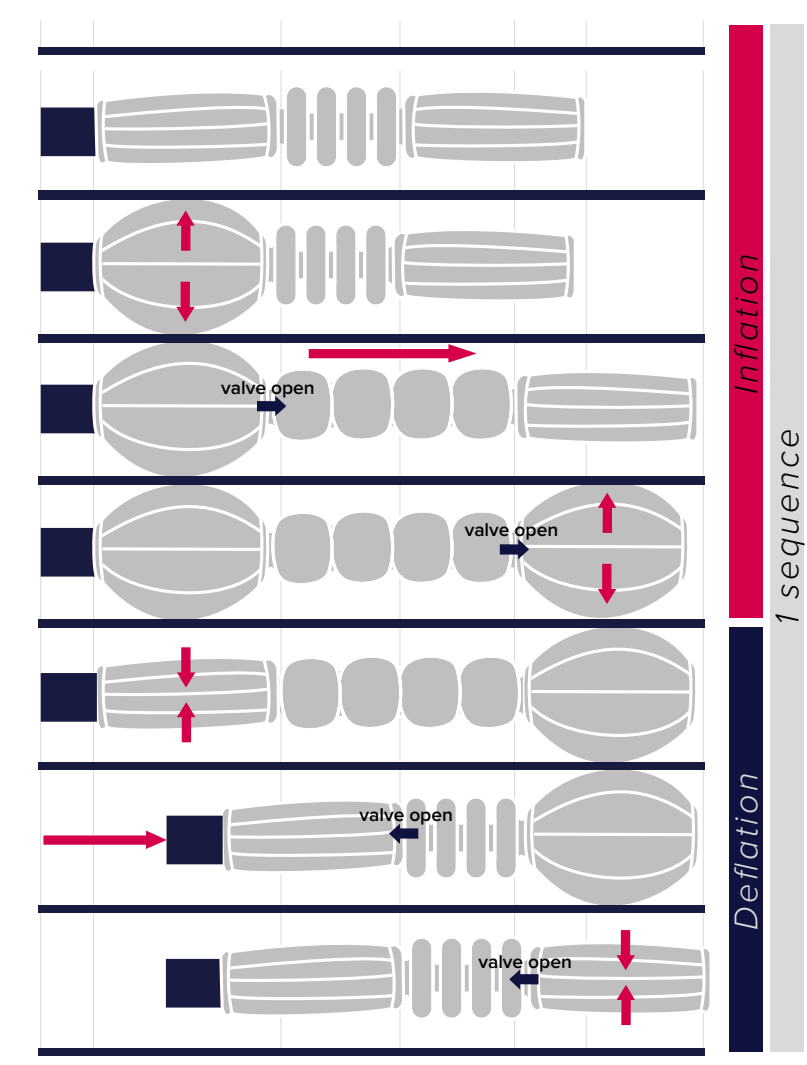

Figure 51. Actuator 5.03 intended actuation sequence.
This print set included 3 prints, all exploring different variations of complex actuation containing multiple geometric sections. The primary goal for this print set was to explore the possibilities of complex actuation. By using different geometric variations, .

determined.

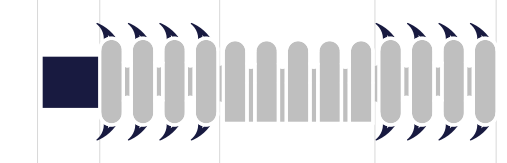

(,,

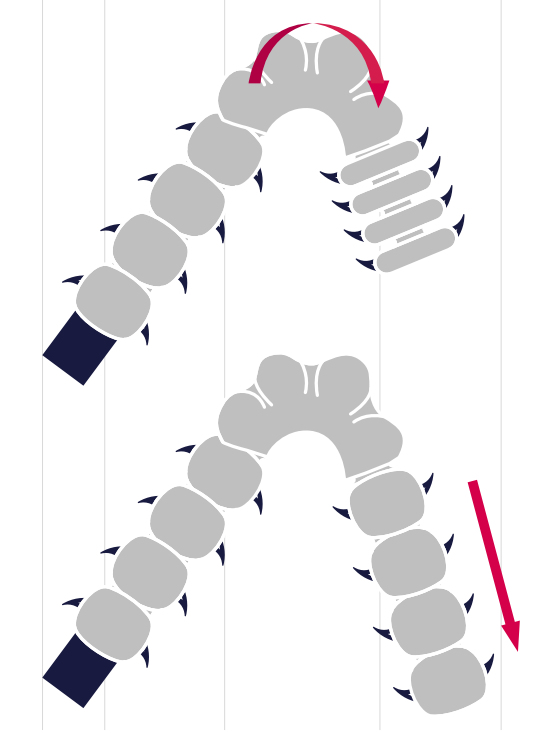

Figure 52. Actuator 5.04 intended actuation sequence. 


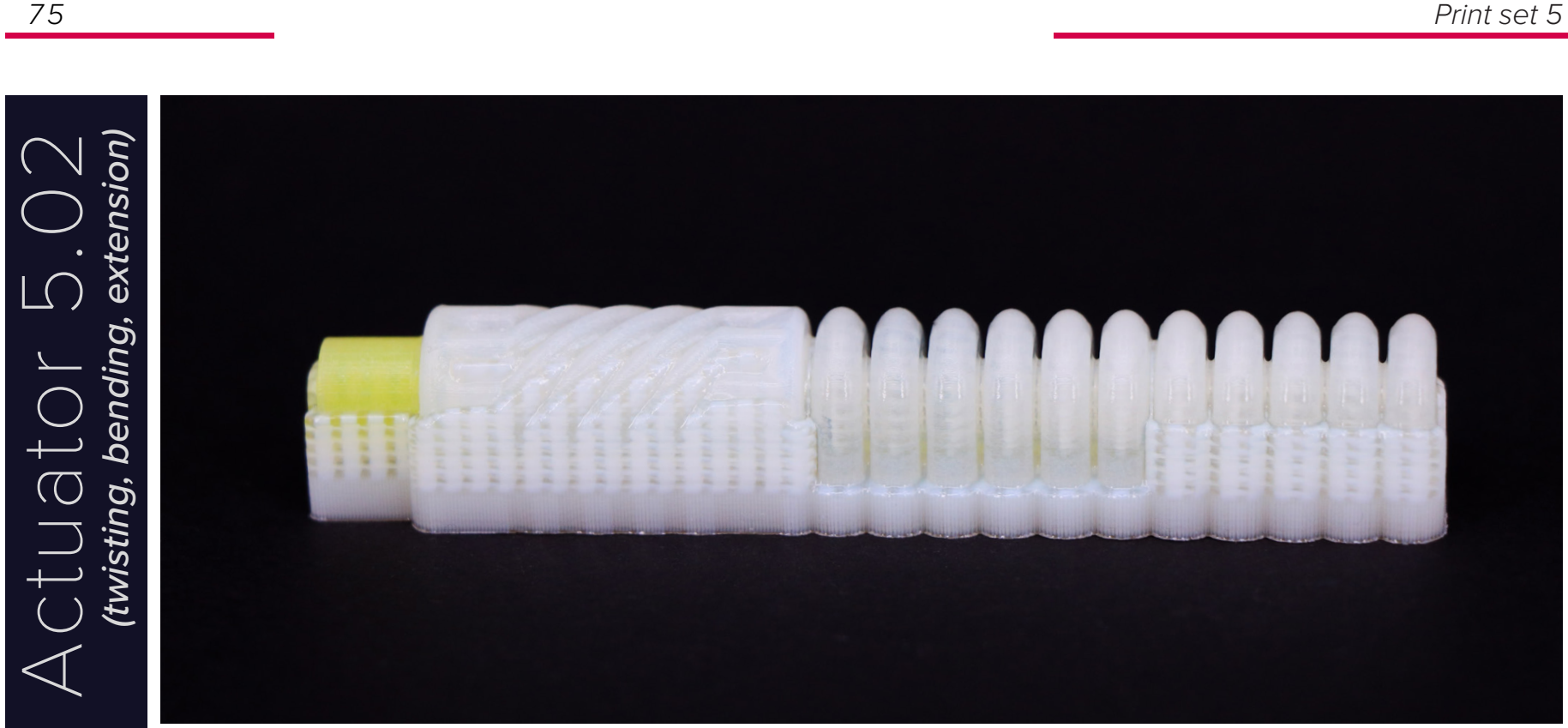

Aim: Create a soft robot capable of achieving multiple motions through one

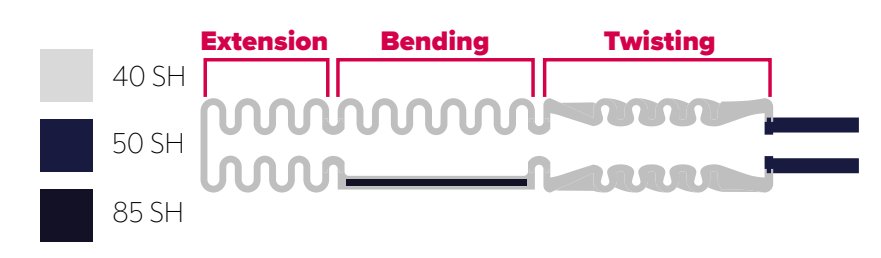

Results: This test confirmed that the twisting section actuates and bursts before the other sections are able to inflate.

Figure 53a - 53b. Actuator 5.02 geometry.
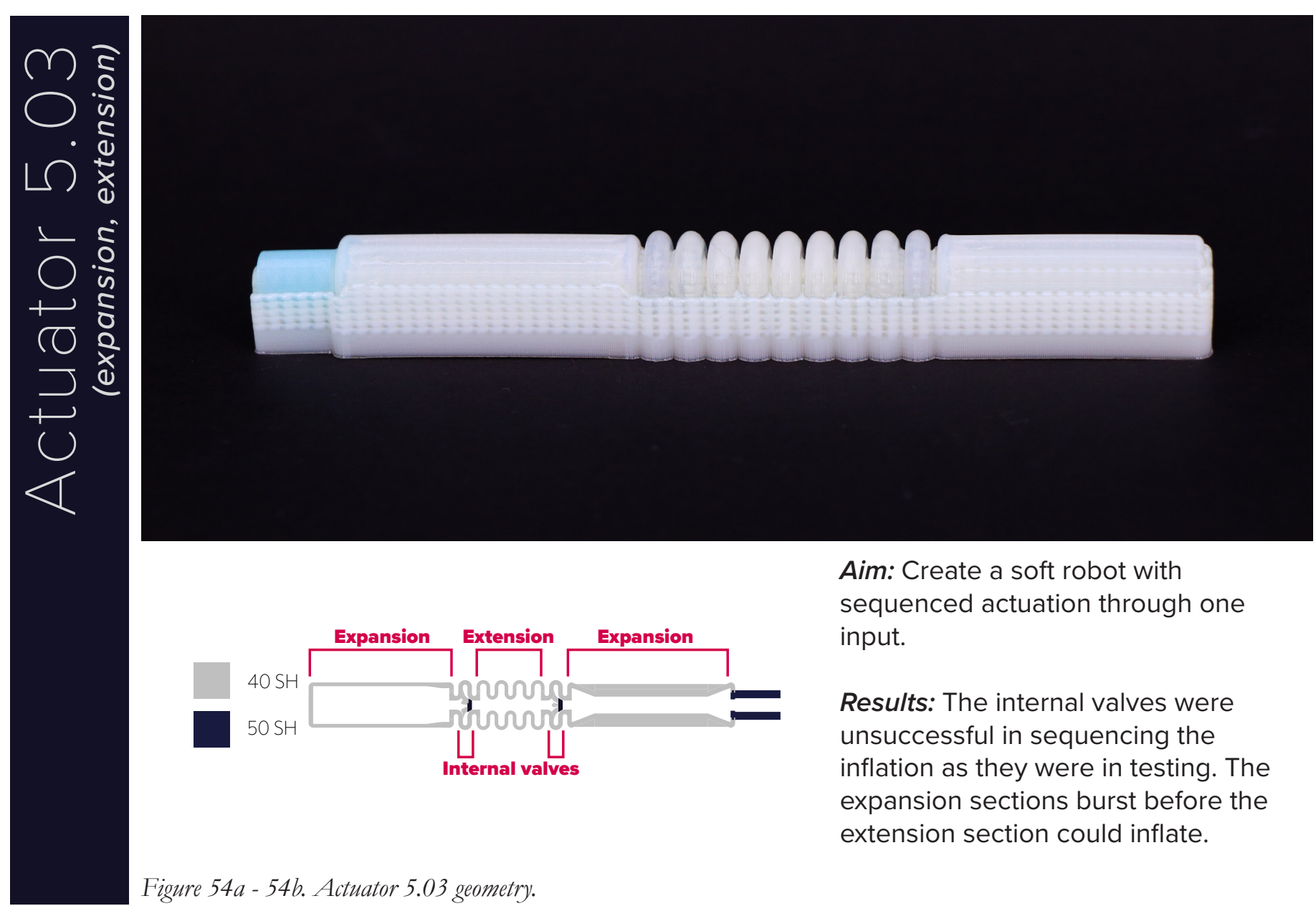

Aim: Create a soft robot with

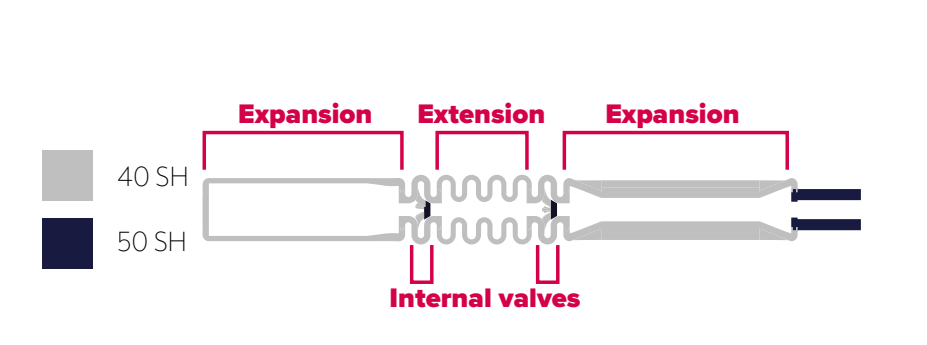

d actuation through one

input.

Results: The internal valves were

unsuccessful in sequencing the

inflation as they were in testing. The

expansion sections burst before the

extension section could inflate.

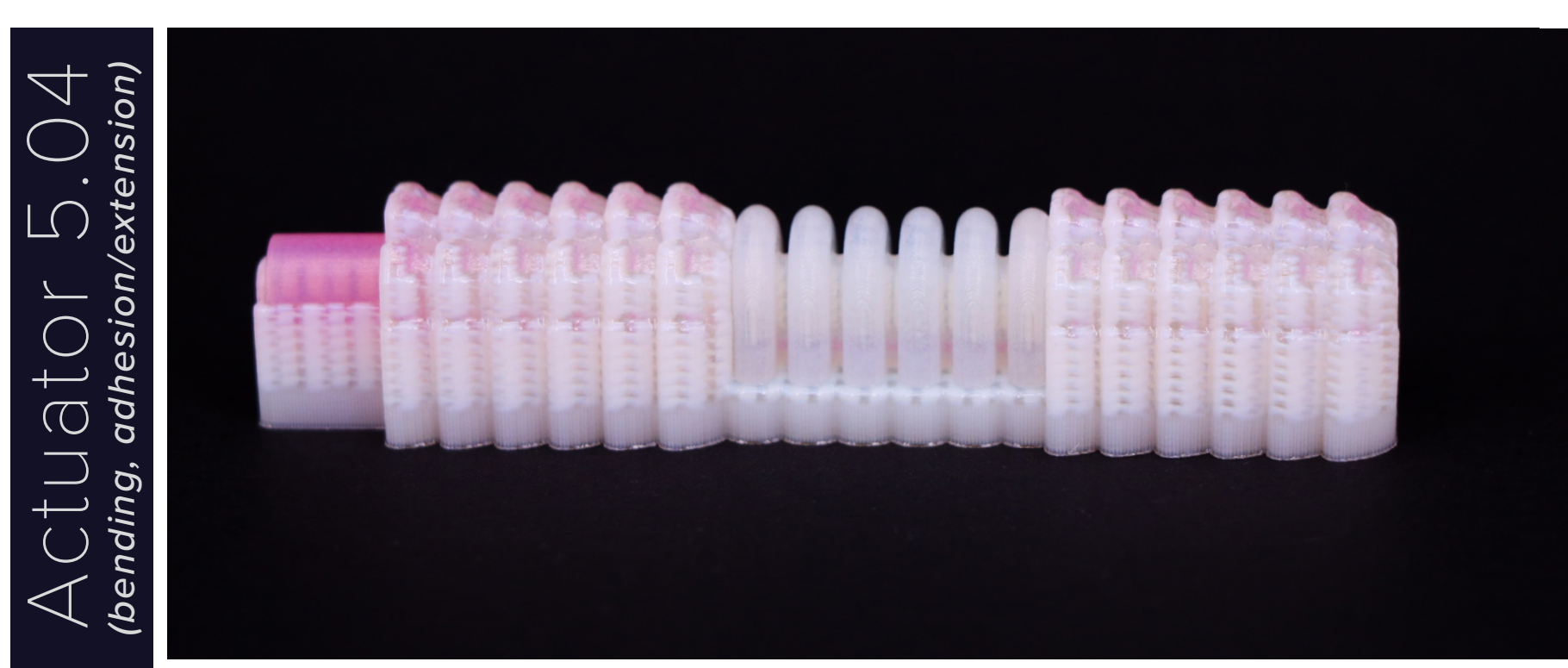

Aim: Create a soft robot capable

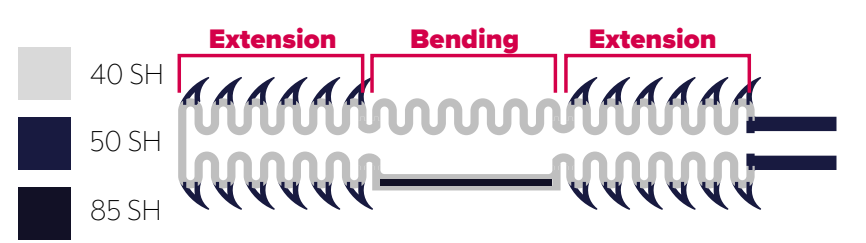
of locomotion by utilising surface adhesion.

Results: All sections successfully inflated, however, the adhesive hooks did not provide enough grip 


\section{Analysis}

The main problem that was identified from this prin set is that the expansion/twisting geometry bursts before a pressure can be reached which inflates the other sections. Actuator 5.04 successfully actuated, as the print did not contain the expansion/twisting geometry. To equalise the pressure needed to actuate a soft robot with both expansion/twisting and extension/bending sections, parameters such as shore hardness and material wall thickness as shore handess and material wall thickness in inflation pressure. The bend section in actuto 5.04 seen to be detrimental to the perfortor 5.04 seemed to be detrimental to the performance of the actuator as it pushed the actuator onto its side rather than bending vertically as was intended. 


\section{Print set 6}

\section{Goals}

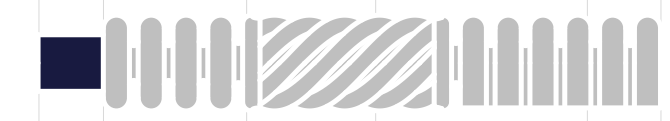

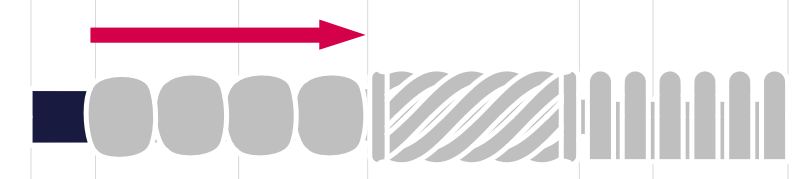

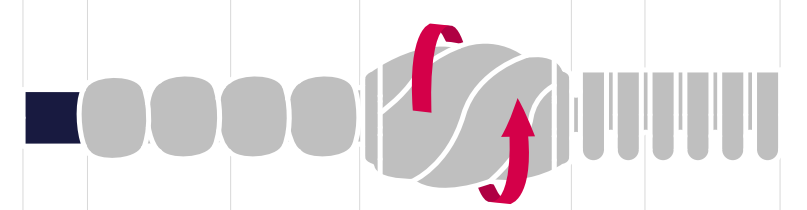

- 00000

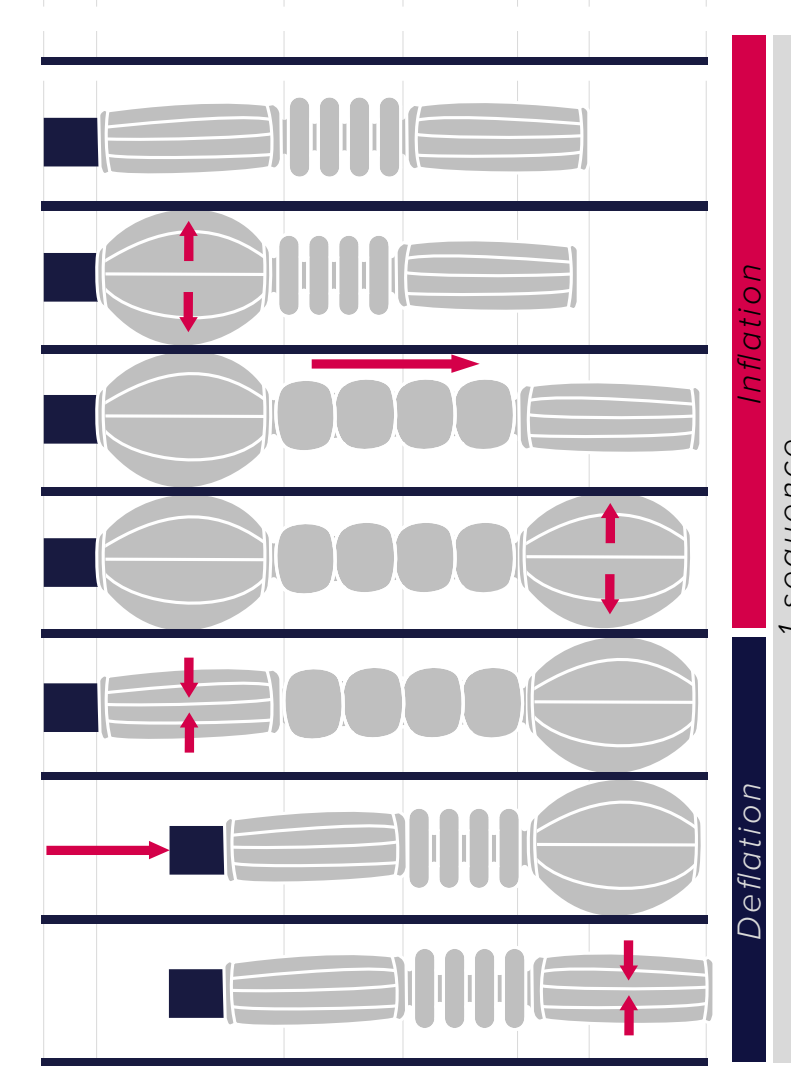

The primary goal for this print set was to successfully actuate three different models that demonstrate complex actuation.

This print set iterated on the previous concepts in an attempt to equalise the pressure needed to actuate the twisting/ expansion geometry and the bending/extension geometry, a couple of changes were made:

1. The shore hardness of the twisting/expansion sections were increased from 40 to 50 .

2. The wall thickness of the bending/extension sections was reduced from $1 \mathrm{~mm}$ to $0.8 \mathrm{~mm}$.

\section{- \\ (O, O,}

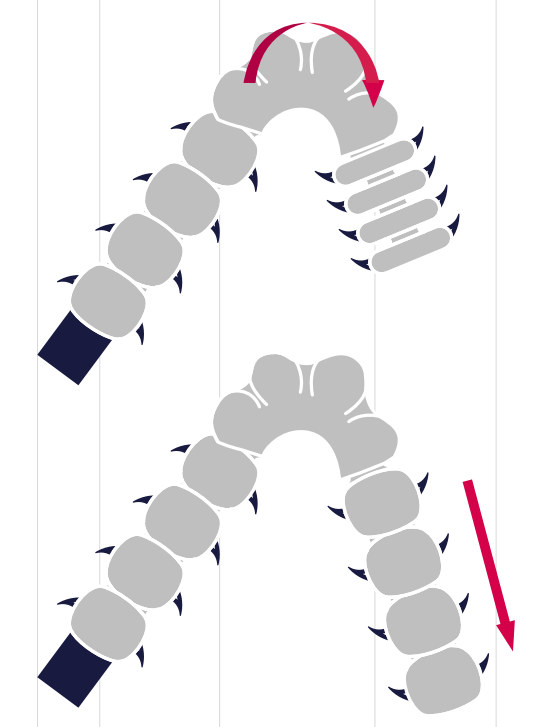

Figure 58. Actuator 5.07 intended actuation sequence. 


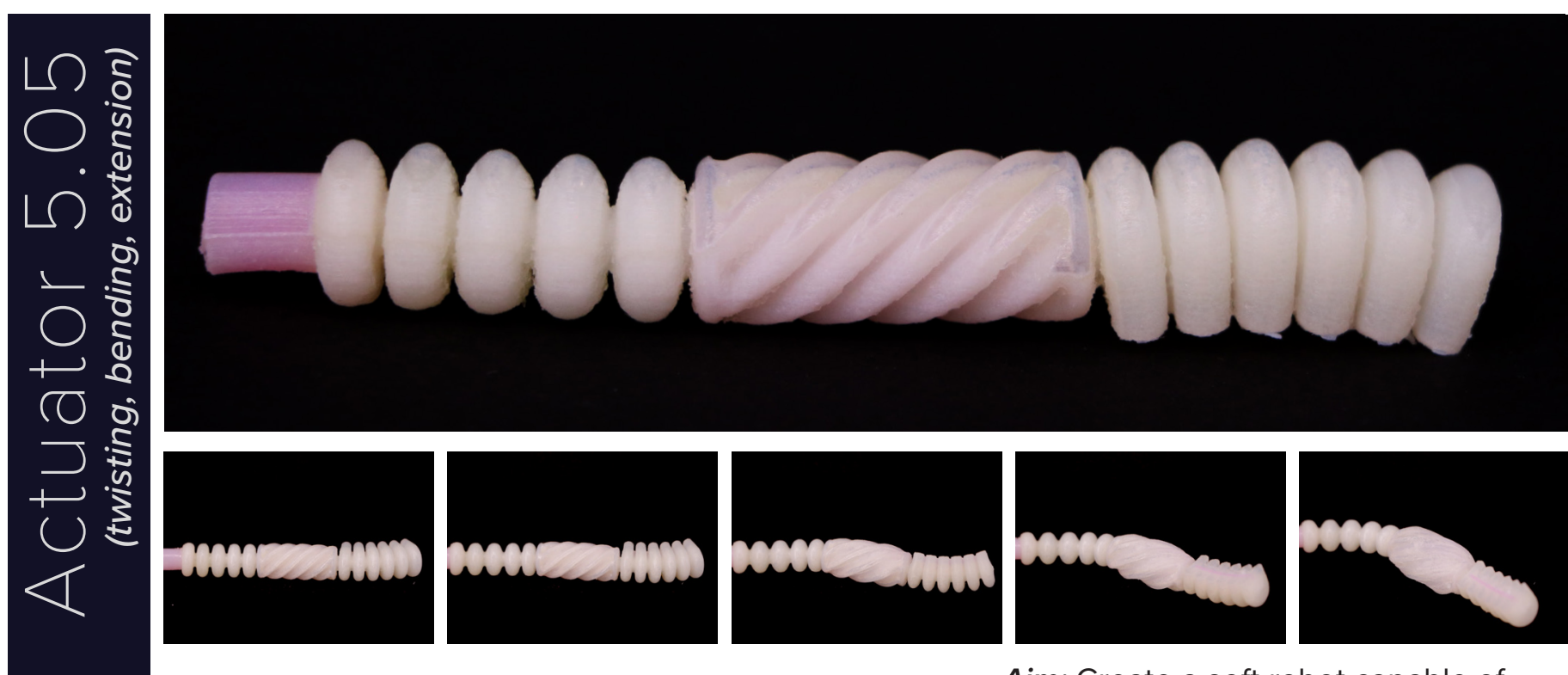

Aim: Create a soft robot capable of achieving multiple motions through one

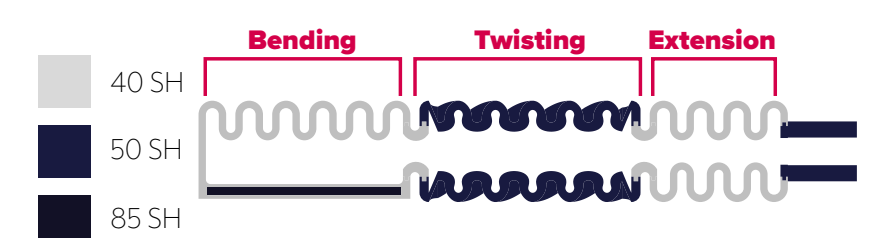

sections successfully actuated. The bending section did not fully inflate due to the twisting section bursting.

igure 59a - 59g. Actuator 5.05 geometry and inflation sequence.

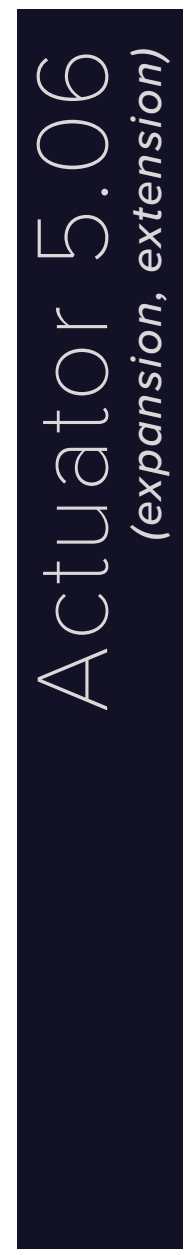

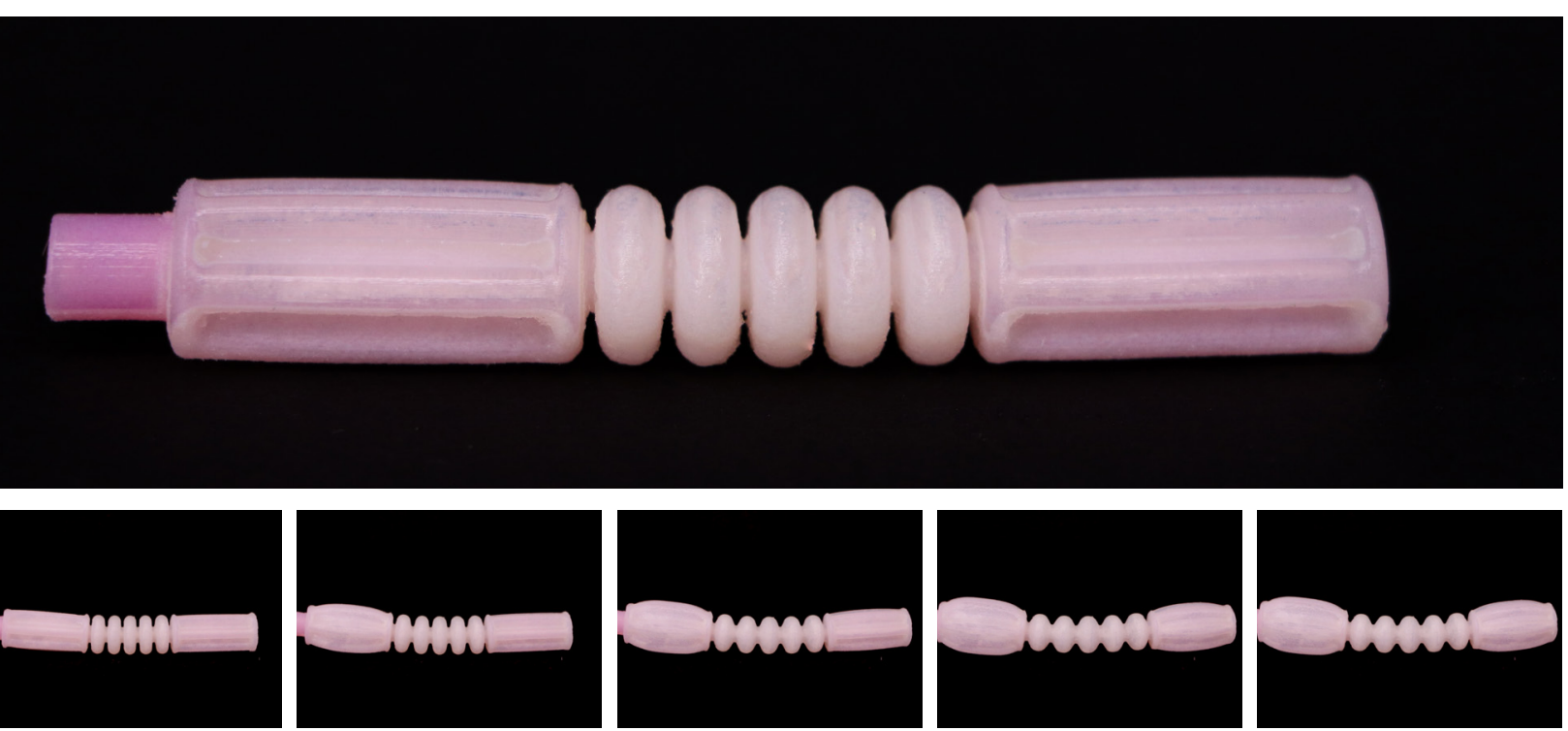

Aim: Create a soft robot capable of

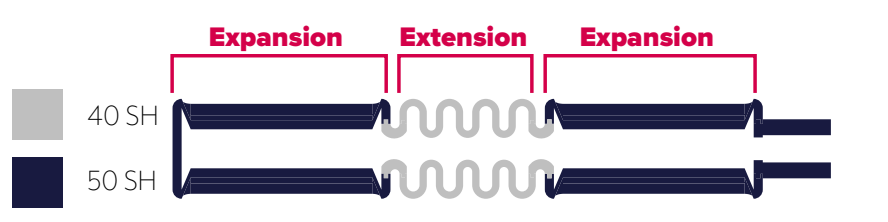

achieving multiple motions through one input.

Results: The print successfully actuated in all three sections, however, the expansion in the second expansion Bursts in the expansion sections limited the success in repeated tests.

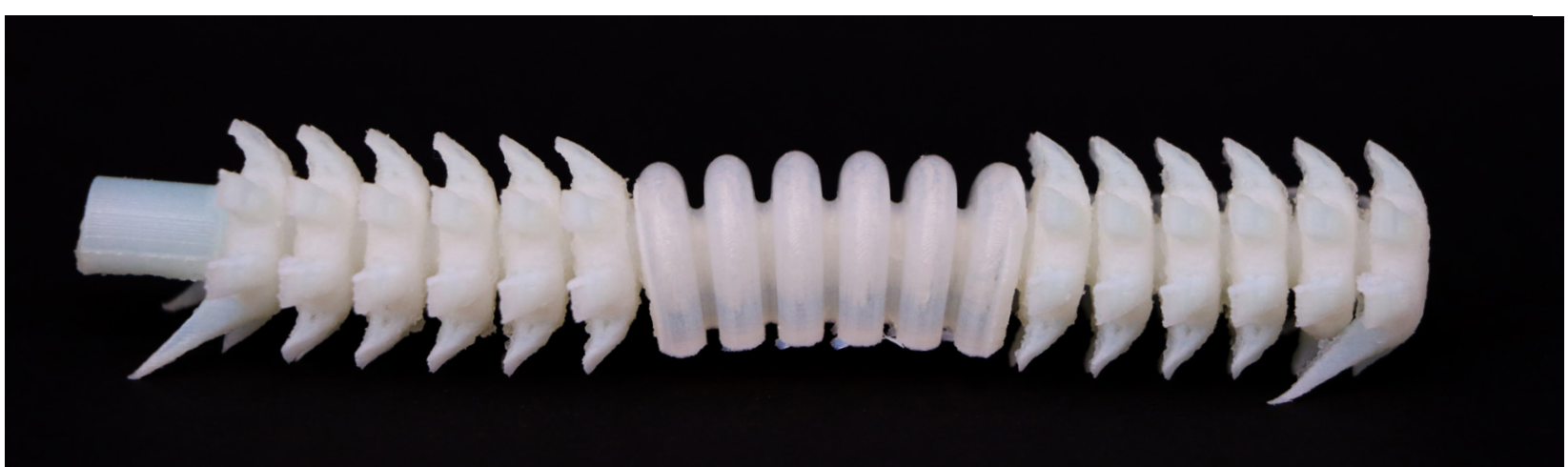

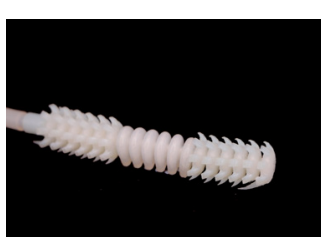
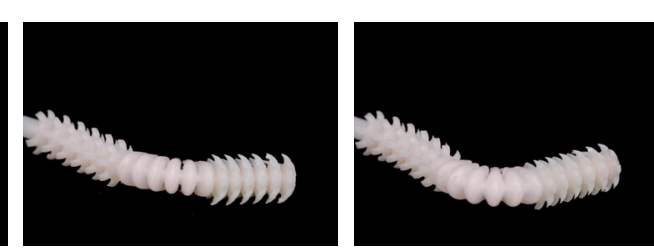

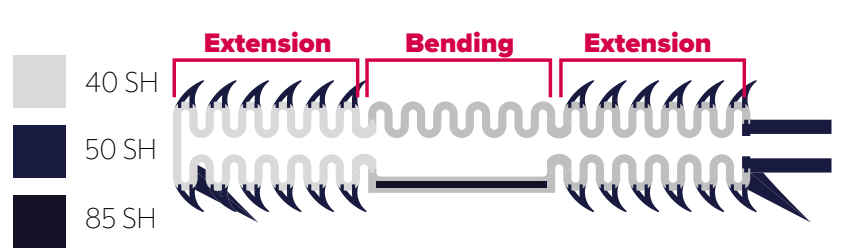

Figure 61a - 61g. Actuator 5.07 geometry and inflation sequence.

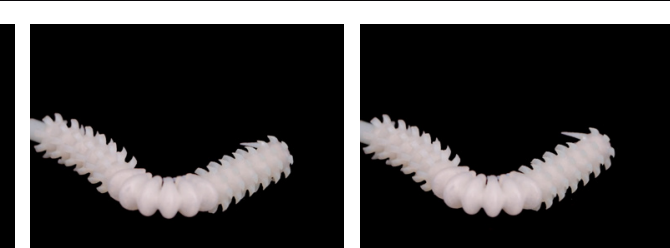

Aim: Create a soft robot capable of locomotion by utilising surface adhesion.

Results: All sections successfully inflated. The addition of longer hooks did not help to orient the print in the intended direction and it still fell onto its side from the bending section inflating. 


\section{Analysis}

The two prints thathadacombination of the bending/ reach its actuation pressure before the twisting extension and twisting/expansion geometry once section inflated and burst. This could mean that again burst during inflation and did not fully actuate. prints with the expansion/twisting sections after Even the changes made in shore hardness and to the bending/extension sections would achieve wall thickness to equalise the pressure across all greater success, however, this would severely the complex cections were pattened appeared to have the fuly actuad however, it was unsuccessul 5.07 sections were patterned appeared to have an fully actuated, however, it was unsuccessful in its effect. In actuator 5.05, the extension section fully movement. The longer hooks at the front and back actuated, but the bending section did not. This is ends of the print designed to keep it upright during ikely because the extension section was before inflation did not work as the print still fell on its side. the twisting section relative to the input, so it could 


\section{Print set 7}

\section{Goals}

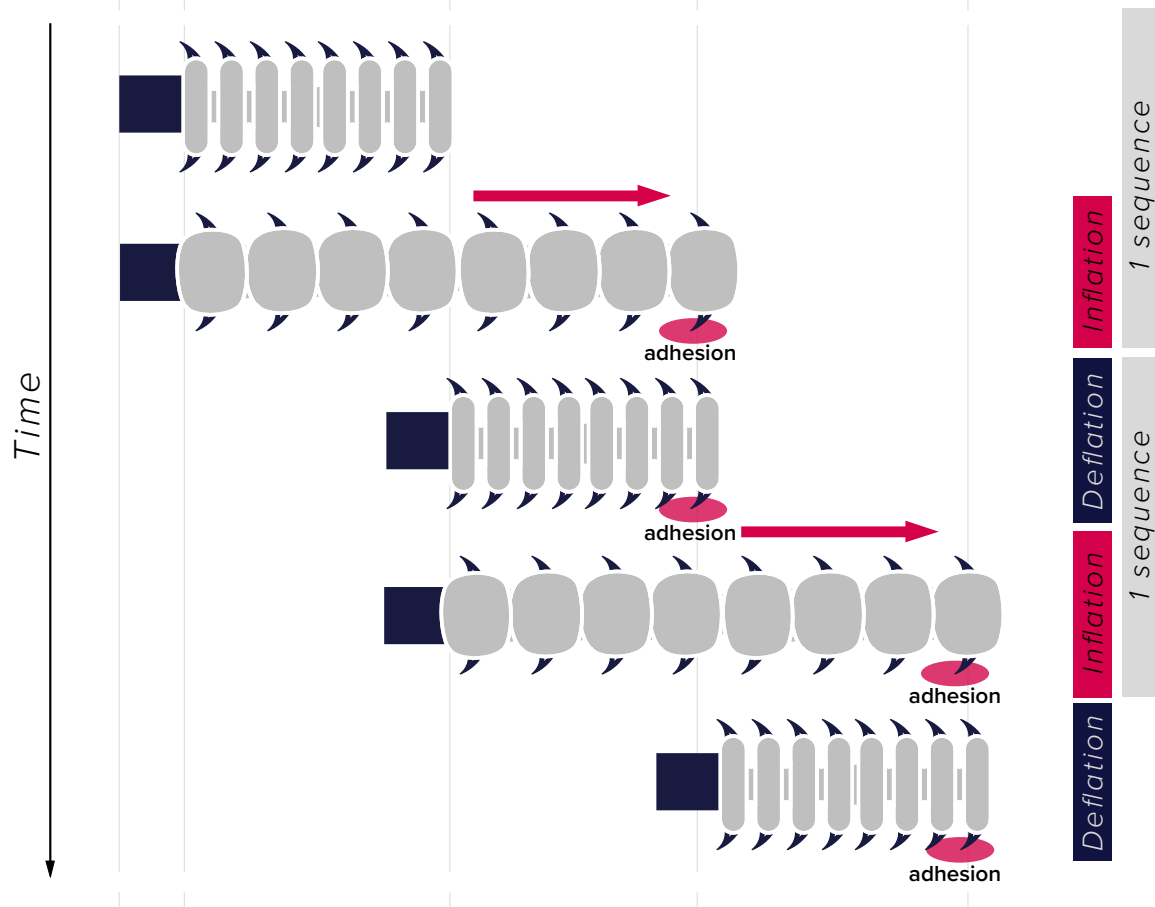

To print a larger scale extension print to compare its durability and performance to other models. This was printed with an increased wall thickness of $1.5 \mathrm{~mm}$.

Print a bending+twisting actuator on a steeper offset angle to compare its performance to the previous bending+twisting print.

Iterate on the original extension+adhesion print

to improve the durability of the adhesive hooks.

A surface was also 3D printed to help the

was also 3 p pinted to help the

extensiontadhesion print successfully achieve

linear locomotion.

Figure 62. Actuator 1.11 intended actuation sequence. 


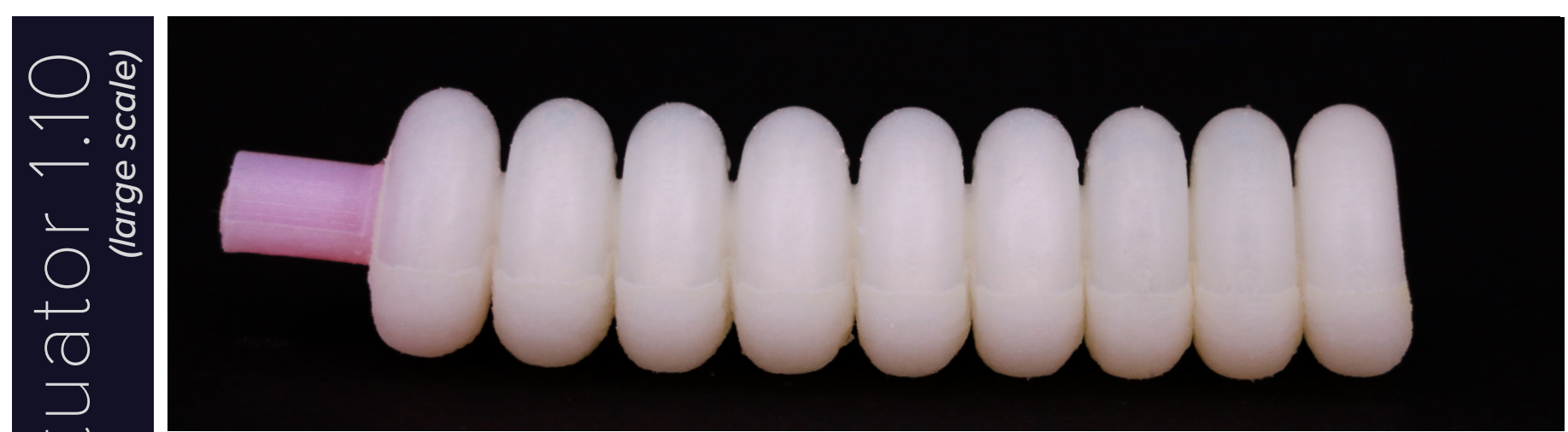

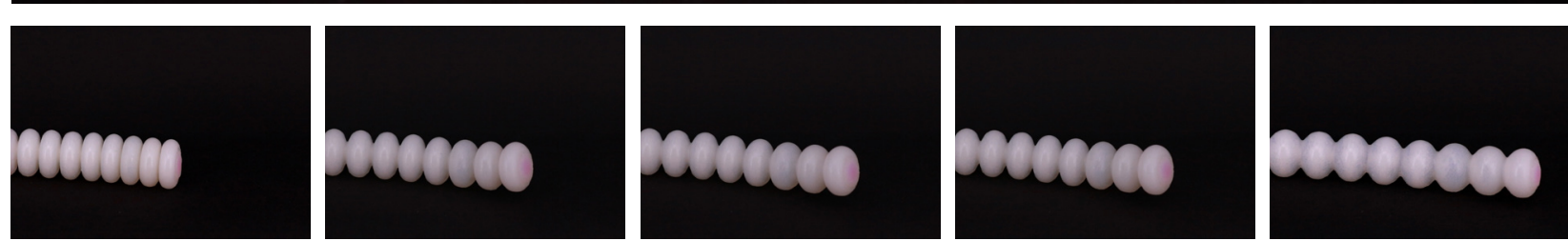

Aim: To assess how scale and wall ¿ ${ }^{40 \mathrm{SH}}$ Aim: To assess how scale and wall
thickness effect on the actuators gth and performance.

Results: The print fully actuated at $7 p s i$ without bursting. An extension percentage of $165 \%$ was reached.

Figure 63a- 63g. Actuator 1.10 geometry and inflation sequence.

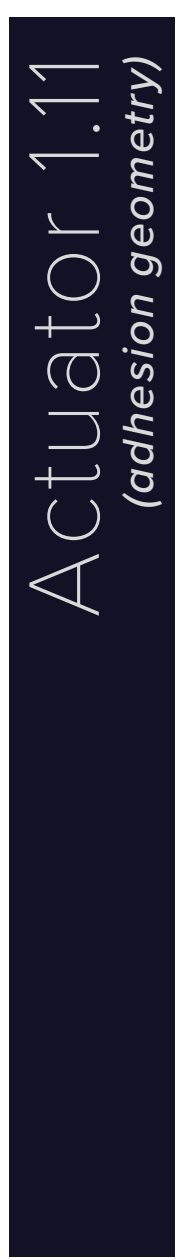

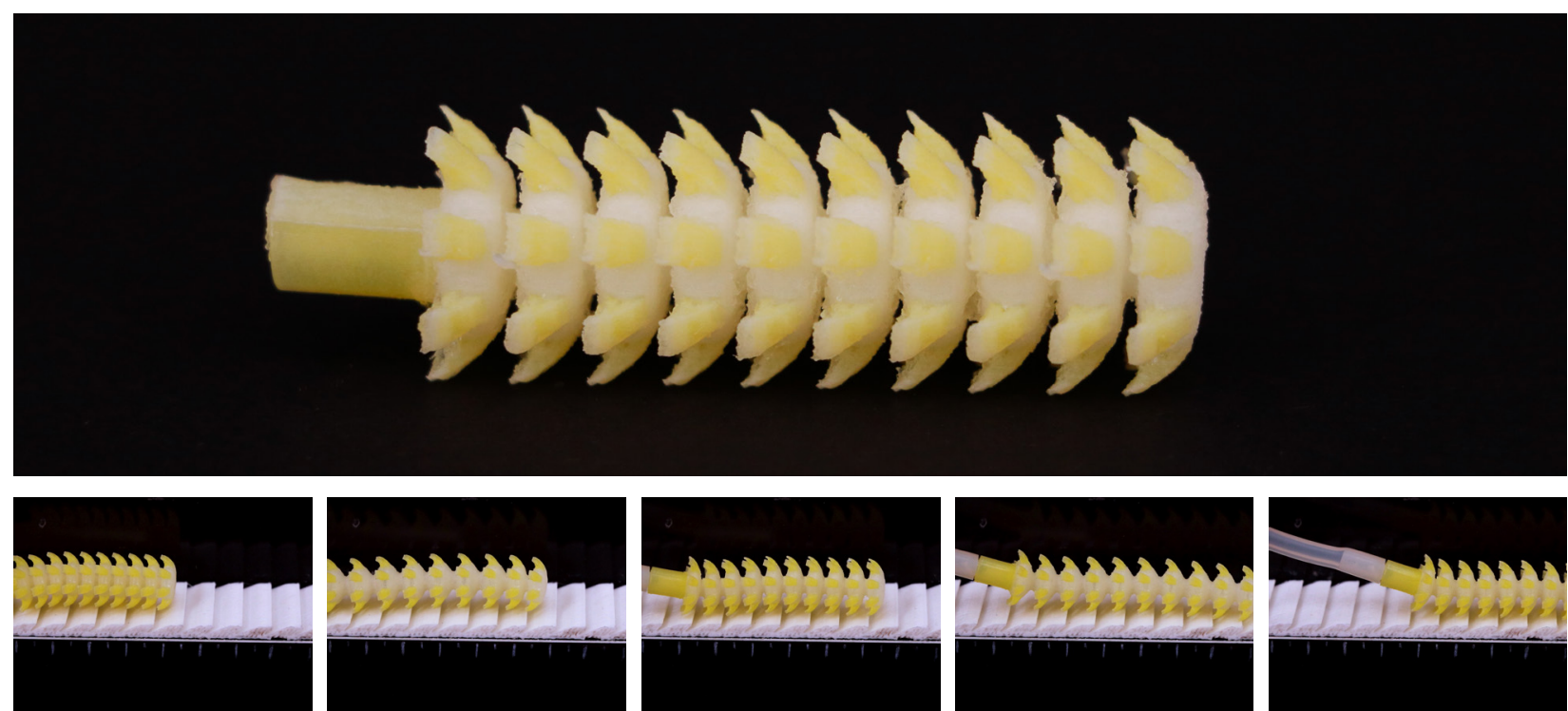

Aim: Create a soft robot capable of locomotion by utilising surface $50 \mathrm{SH}$
$85 \mathrm{SH}$

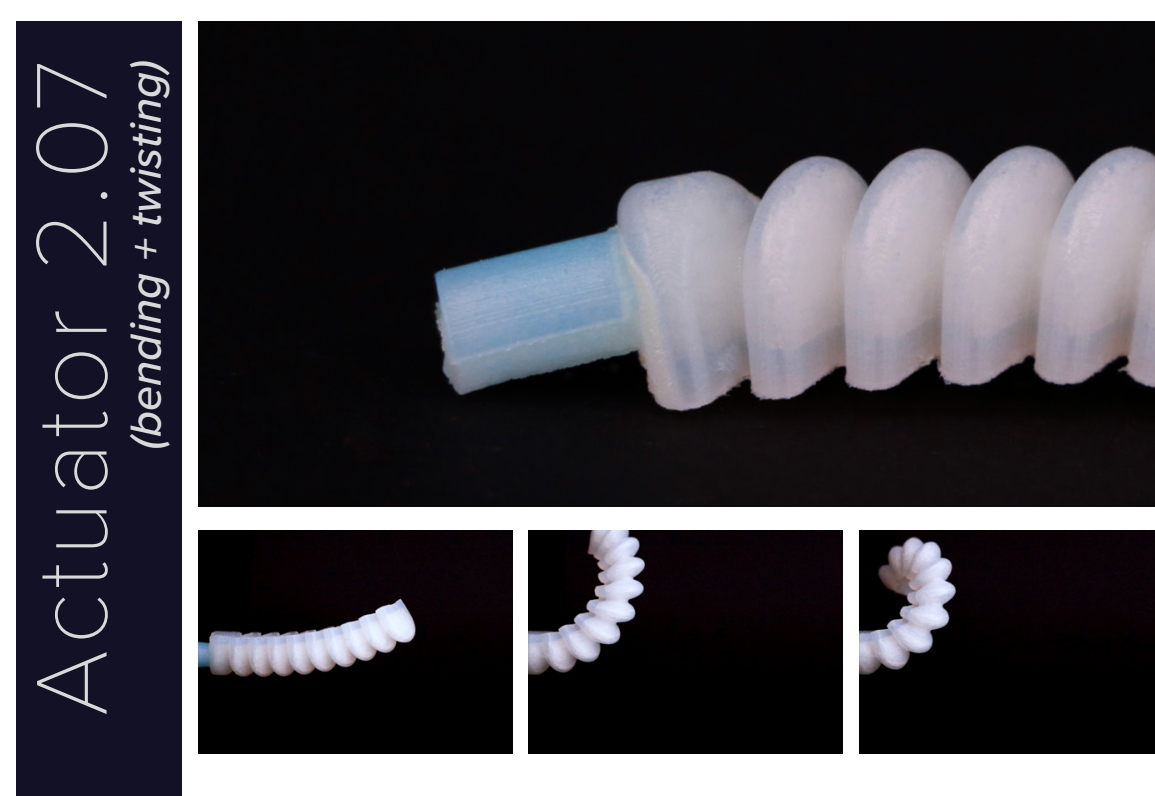

- ${ }_{50 \mathrm{SH}}^{20 \mathrm{H}}$

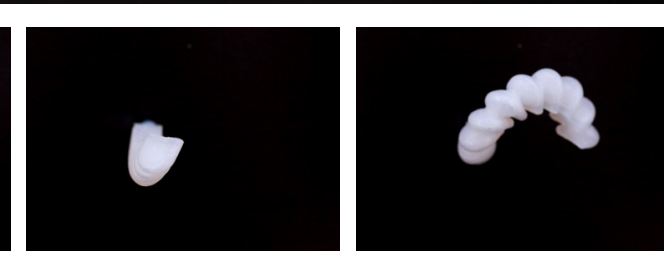

Aim: Assess the effect of the bellow offset angle on the actuator's

ability to follow a helical path upon inflation.

Results: The print successfully followed a helical path, and demonstrated a steeper helix angle to the $25^{\circ}$ bellow offset print.
Figure 65a - 65g. Actuator 2.07 geometry and inflation sequence. 


\section{Analysis}

Scaled extension actuator (1.10):

This print demonstrated much better durability to previous extension prints. This was evident from its high actuation pressure and its ability to hold the pressure. When this actuator was deflated, it took pressure. When this actuator was deflated, it took
much longer for the actuator to depressurise than

much longer for the actuator to depressurise than

the other extension actuators, which could indicate
that the air is only being let out through the pump,

whereas, with the smaller extension pints, faster

depressurisation could have occurred due to smal

tears in the print allowing air to escape.

\section{Extension/adhesion actuator (1.11):}

This was the first print to successfully demonstrate linear locomotion. By calibrating the pressure limit of the pump to the maximum inflated state of the actuator, the pump pulsed its airflow, allowing the actuator to inflate and deflate repeatedly. The adhesion geometry on the actuator was able to successfully hook onto the adhesive surface, which pulled the actuator forward when it was deflated and this was repeated to achieve locomotion. The actuator moved at $2.3 \mathrm{~mm} / \mathrm{s}$, which was increased in later testing to $2.9 \mathrm{~mm} / \mathrm{s}$ by decreasing the space between the hooks in the adhesive surface.

\section{Bending + twisting actuator (2.07):}

The increase of the bellows offset from the previous bending + twisting print had a clear impact on the helix angle that the print followed during actuation. When the offset angle of the bellows is increased so is the offset of the actuation from the primary axis of bend. 


\section{Print set 8}

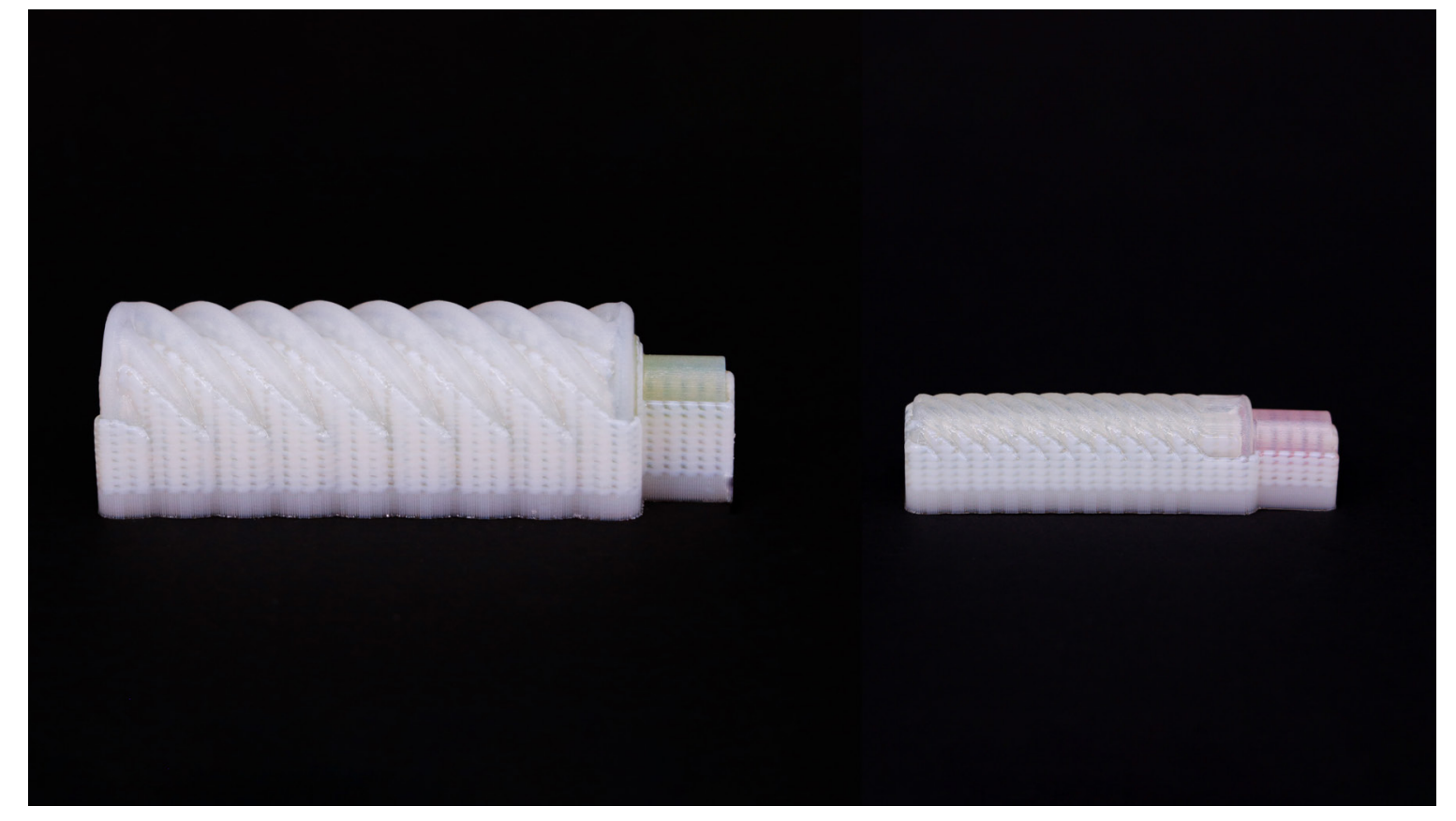

Figure 66. Difference in size of large scale prints.

\section{Goals}

This print set tested two actuators at a larger scale The second goal of this print set was to re-evaluate for twisting and expansion. Like the bending test, the internal valve geometry so that it was more the wall thickness was increased to $1.5 \mathrm{~mm}$. The compatible with the print resolution. To do this, goal was to determine whether the increase of the complex geometry was replaced with a simple wall thickness would allow the print to actuate at a hole in the middle of two extension sections. The wall thickness would allow the print to actuate at a hole in the middle of two extension sections. The
pressure comparable to the scaled bending print.
diameter of the hole was given three separate $\begin{array}{ll}\text { pressure comparable to the scaled bending print. } & \begin{array}{l}\text { diameter of the hole was given three separate } \\ \text { values, to determine the pressure at which the hole }\end{array}\end{array}$ would allow air to pass through. 

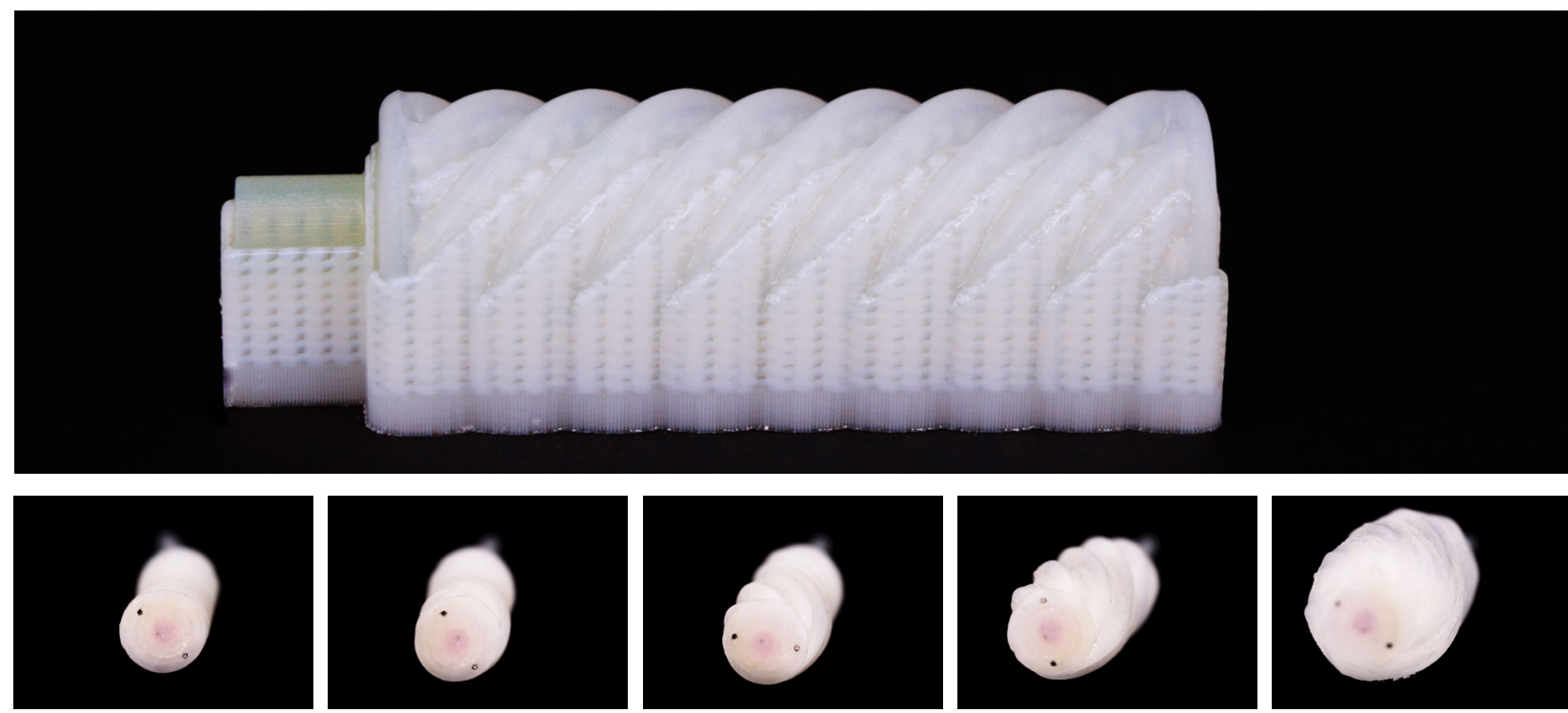

Aim: To assess how scale and wall Aim: To assess how scale and wal ${ }_{50 \mathrm{SH}}^{40 \mathrm{SH}}$ strength and performance.

Results: Small geometric gaps in the lofts at the ends let air out in the first tests. Once these were patched with glue, the print burst along one of the inner radii at $5 \mathrm{psi}$. A twist angle of $5.0^{\circ}$ Figure 67a- 67g. Actuator 3.11 geometry and inflation sequence. $\mathrm{mm}\left(258^{\circ}\right.$ total)was reached.
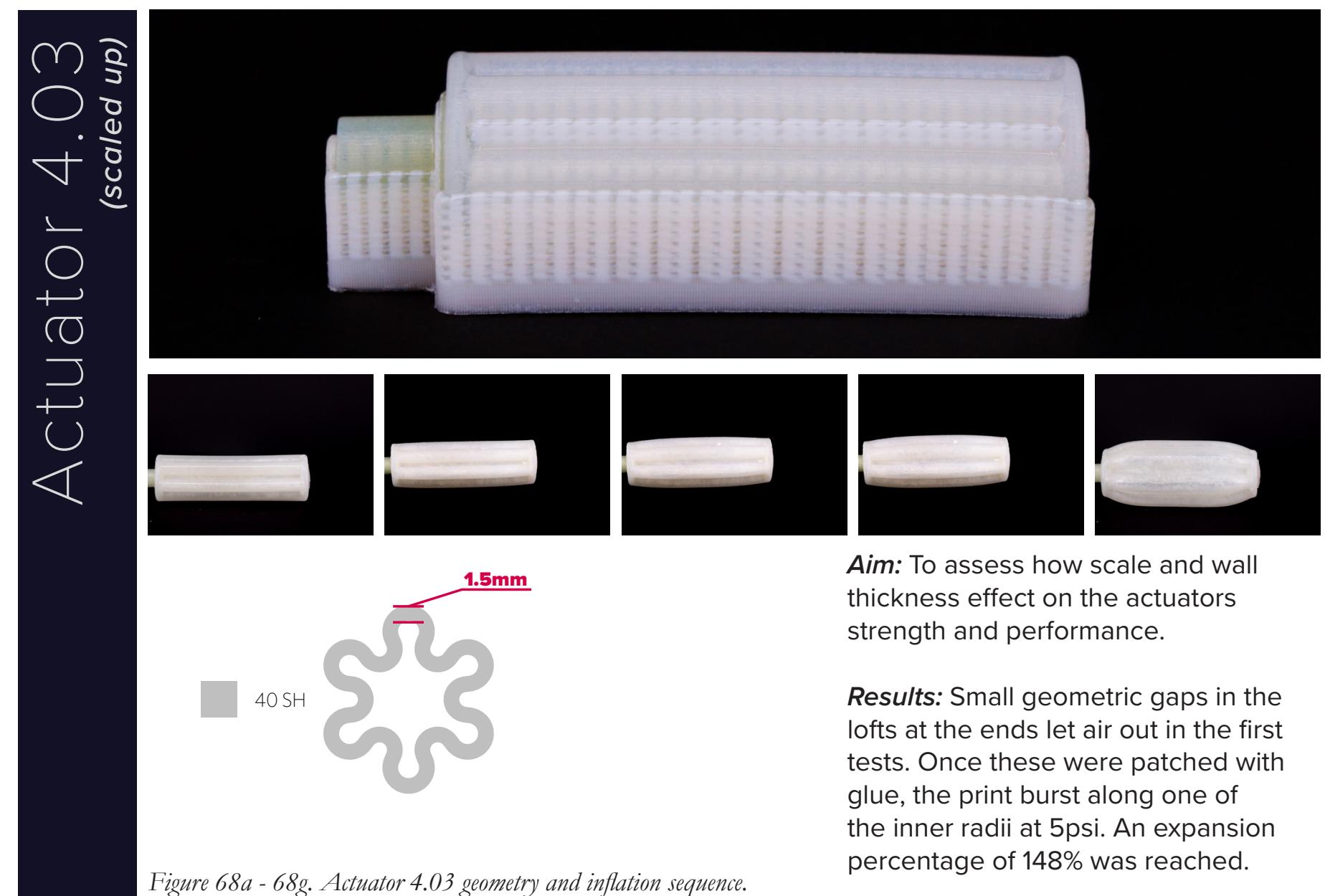

Aim: To assess how scale and wall

thickness effect on the actuator

strength and performance.

Results: Small geometric gaps in the lofts at the ends let air out in the first tests. Once these were patched with glue, the print burst along one of the inner radii at 5 psi. An expansion percentage of $148 \%$ was reached.

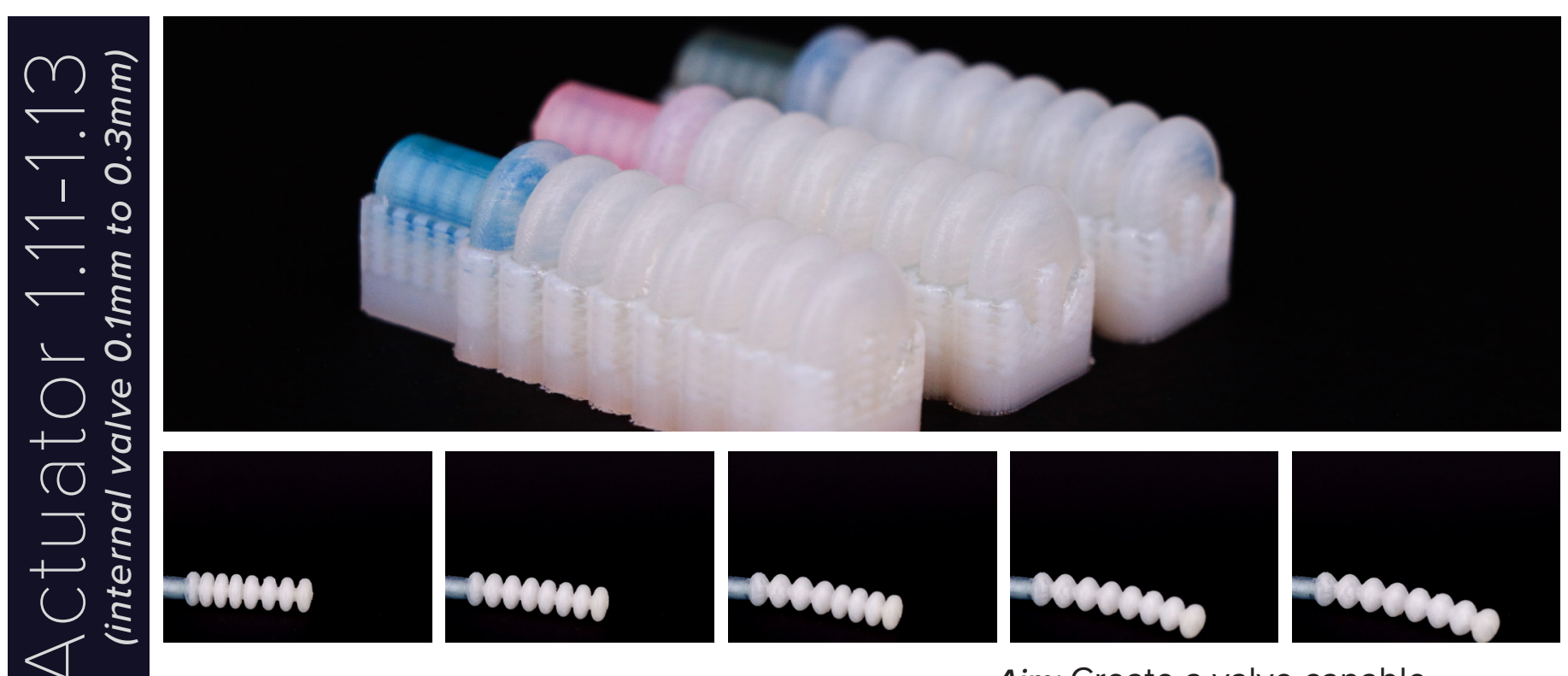

Aim: Create a valve capable ש5 actuation.

Results: $0.1 \mathrm{~mm}$ and $0.2 \mathrm{~mm}$ valve holes did not let any air through up to 10 psi. The $0.3 \mathrm{~mm}$ valve hole let air through to the second section at $4 \mathrm{psi}$. 


\section{Analysis}

The large scale prints in this set did not yield much success. For them to be a success, they needed to actuate at the same pressure as the extension geometry at the same scale. As the extension print required $7 \mathrm{psi}$ to fully actuate and the large print prints in this set burst at $5 \mathrm{psi}$, this was dee scale failure.

The pressure relief valves yielded some success, where the $0.3 \mathrm{~mm}$ valve managed to hold pressure until reaching $4 \mathrm{psi}$, at which point the air flowed through to the second half of the actuator and it fully inflated. The main issue with this, however, was that when the actuator was retested after the initial test, the valve no longer restricted the airflow and the actuator fully inflated without successul ac sequencing. This was likely due to the valve being bequen blocked by material until it reached 4psi initially, and after the material had been removed the ai flowed freely through the valve. 


\section{Print set 9}

\section{Goals}

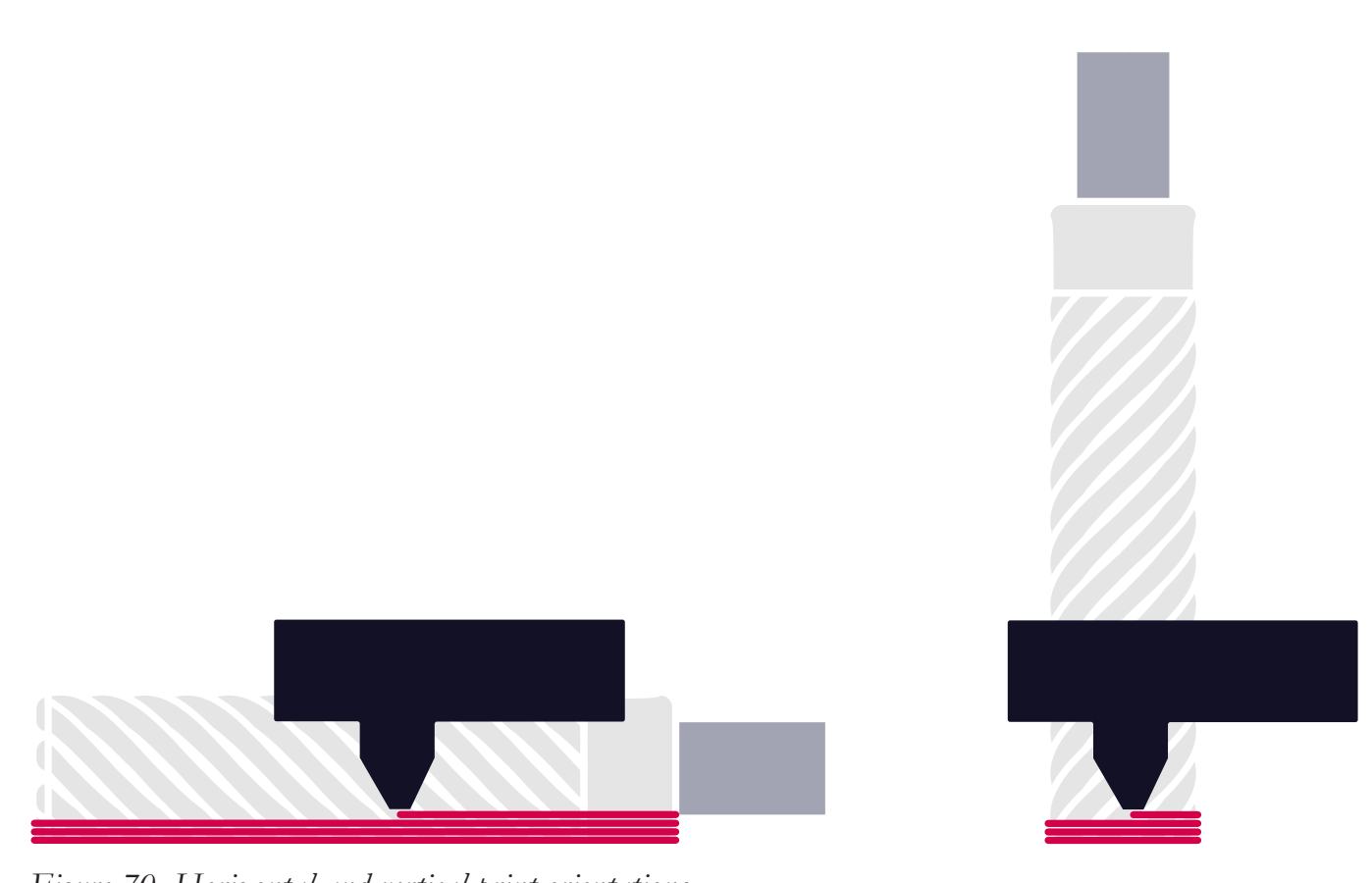

This print set aimed to explore how the print orientation of the models on the Stratasys $J 750$ would impact their performance. Both the twisting and the expansion geometry prints were printed

laying horizontally and standing vertically on the

print bed (Figure 70). These models were chosen

as they are the models that burst at the lowest

as they are the models that burst at the lowest

pressures across the experimentation. To test the

pressure at which they burst, the pump's maximum

pressure was increased in $0.5 \mathrm{psi}$ increments.

Figure 70. Horizontal and vertical print orientations. 


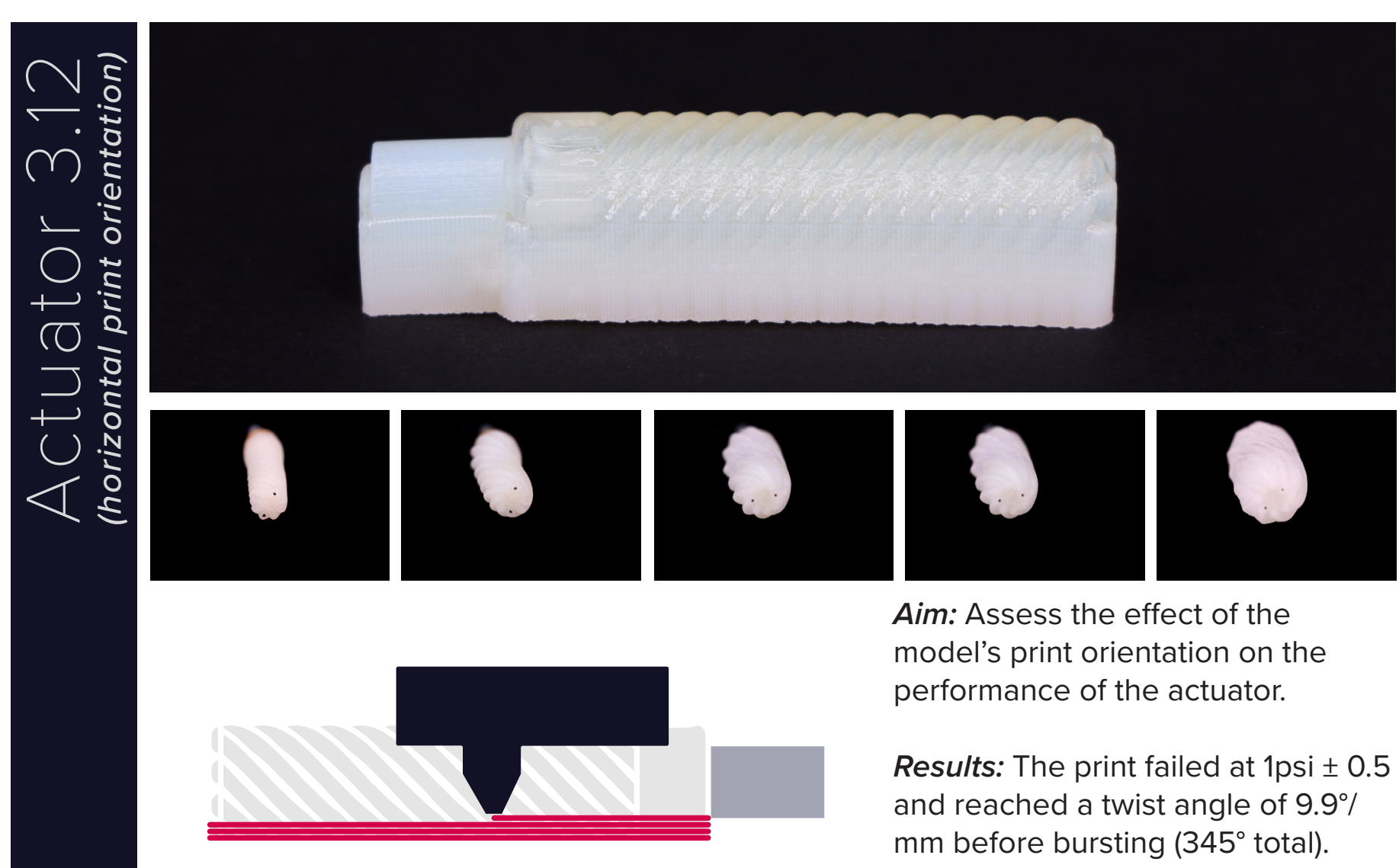

Figure 71 a - 71g. Actuator 3.12 geometry and inflation sequence.

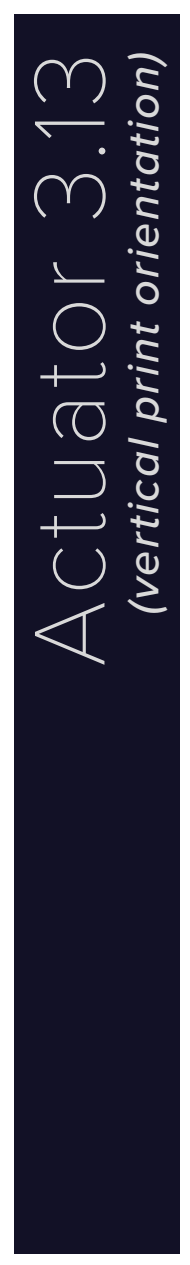

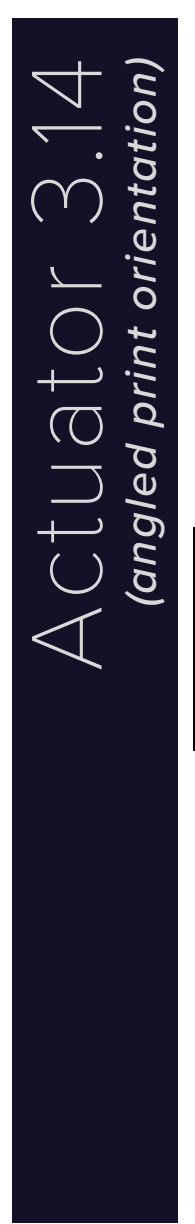
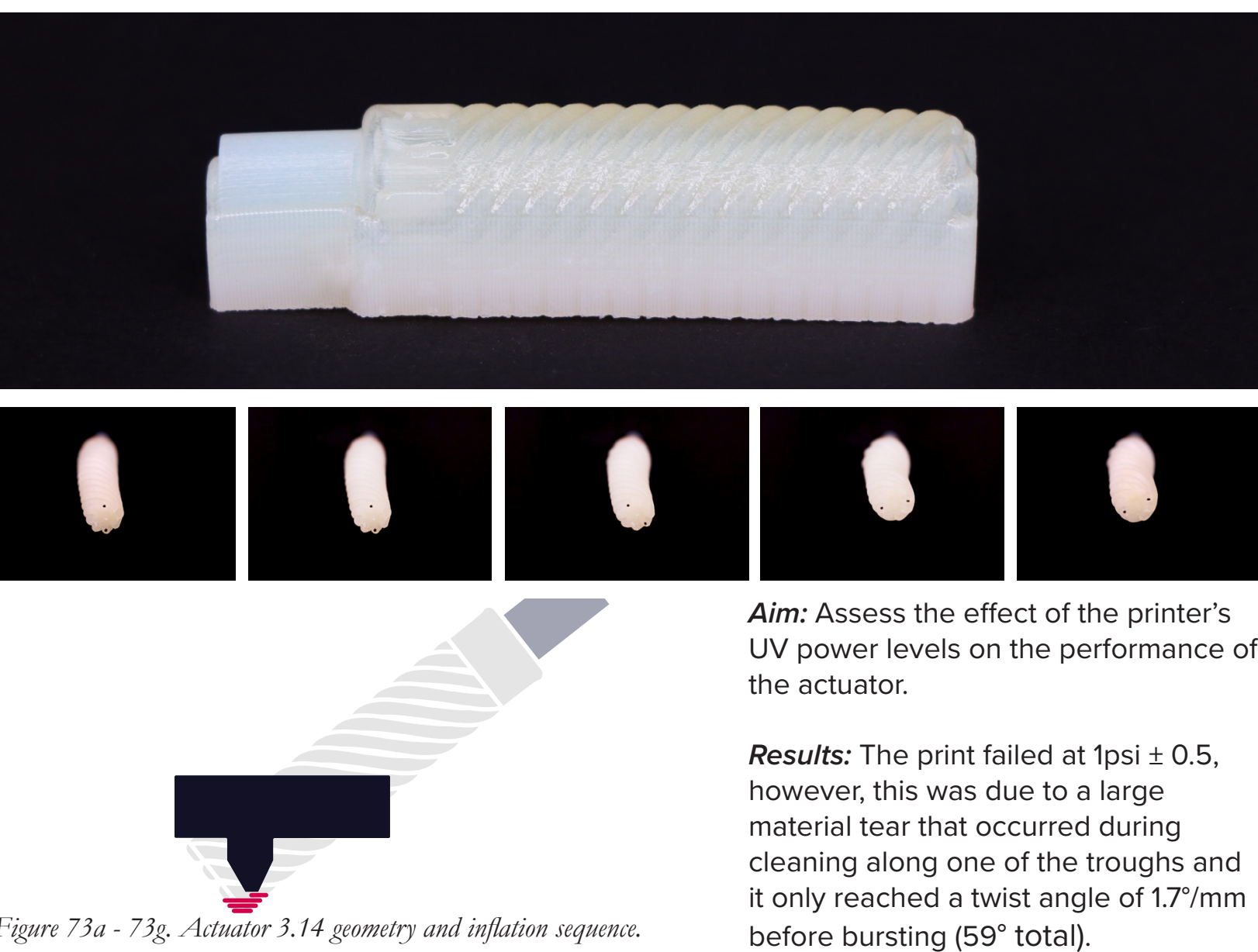

Aim: Assess the effect of the printer's UV power levels on the performance of the actuator.

Results: The print failed at $1 \mathrm{psi} \pm 0.5$,

however, this was due to a large

material tear that occurred during

cleaning along one of the troughs and it only reached a twist angloughs and Figure $73 a-73 g$. Actuator 3.14 geometry and inflation sequence. before bursting $\left(59^{\circ}\right.$ total).

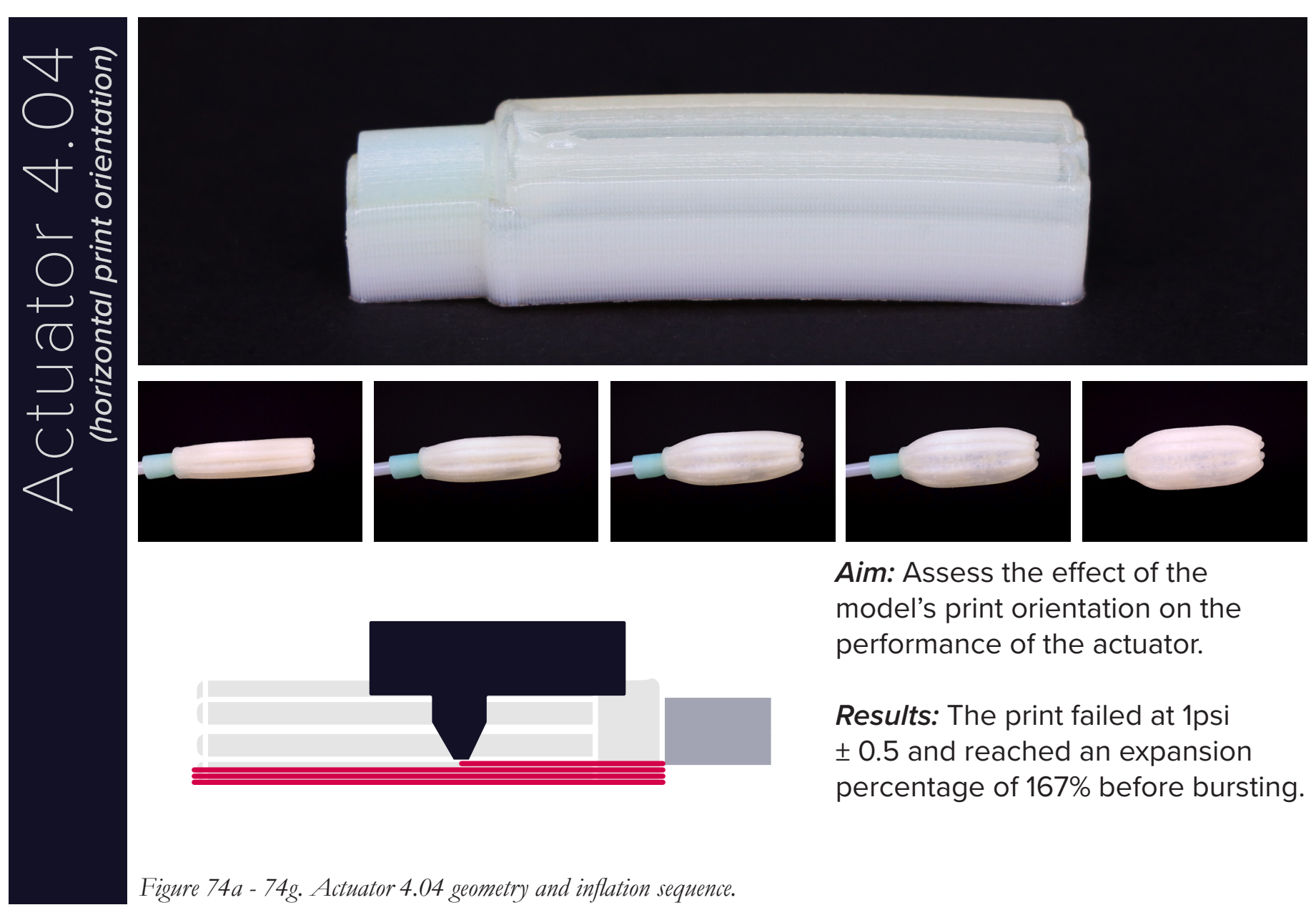



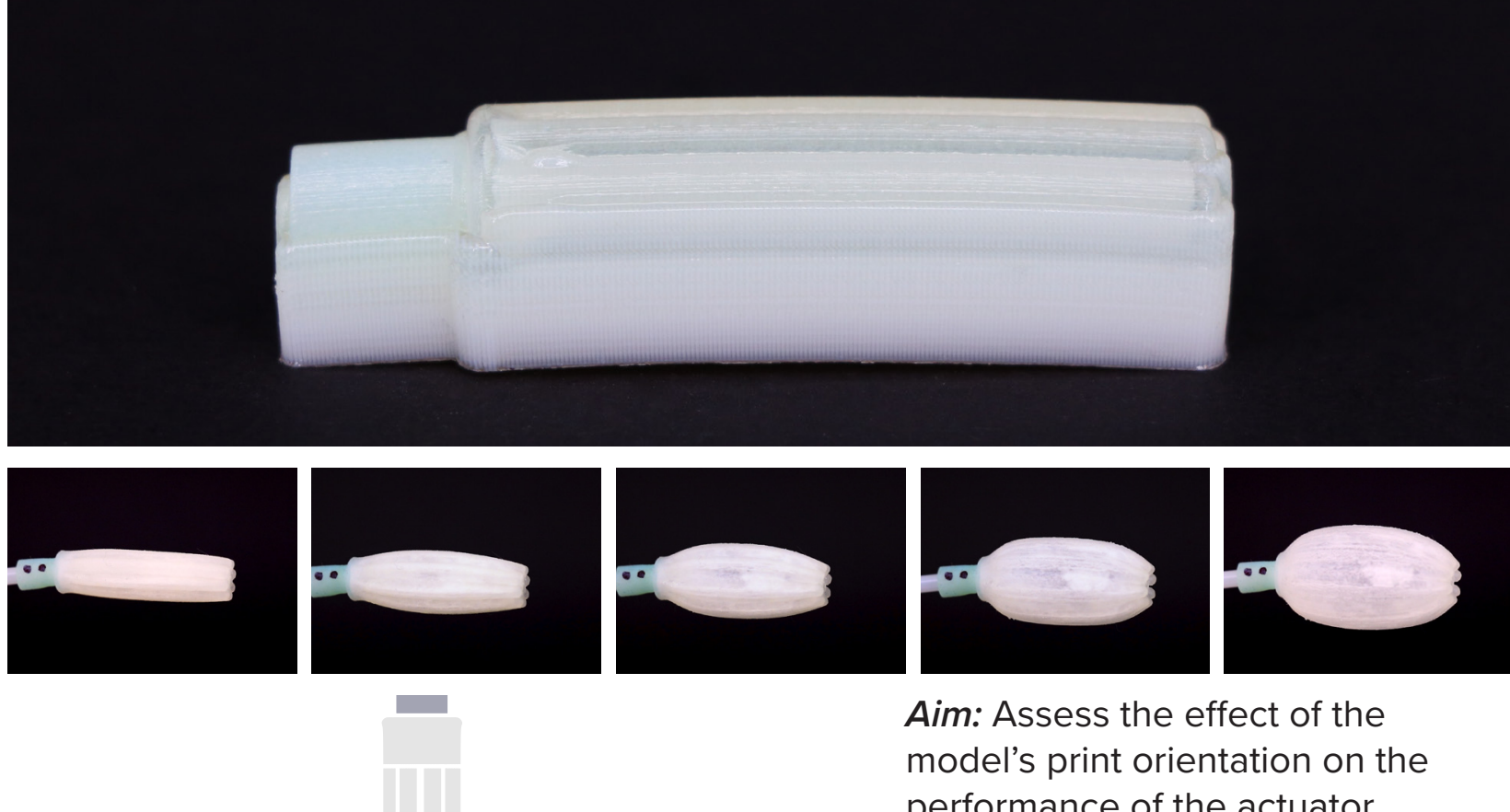

\section{Analysis}

Results: The print failed at $1.5 \mathrm{psi}$

\pm 0.5 and reached an expansion

percentage of $212 \%$ before bursting

Although the accuracy of the pressure testing twist percentage of the vertically and horizontally isn't high enough to draw conclusions at these oriented prints also supports this. The horizontally pressures, there was a clear difference in the oriented print showed a slightly larger twisting expansion percentage between the vertically and percentage than the vertically oriented print $\left(14^{\circ}\right.$ horizontally oriented expansion prints. A similar larger). For the vertical orientation print, the laye result appeared to occur in the twisting prints, print direction is offset by $35^{\circ}$ from bellows angle, however, the expansion percentage was not whereas for the horizontal orientation the layer recorded. The angled orientation twisting print print direction is offset by $55^{\circ}$ from the bellows (3.14) exhibited a larger weakness during cleaning angle. From these results, it was concluded that than either the vertically (3.13) or horizontally the bellows that are at a larger offset angle from (3.12) oriented prints, which suggests that having the print layers tend to be less prone to tearing and the troughs align with the print direction does the the optimat print orientation is when the they're negative in is also supported by the difference in expansion orientation of the models does have an impact on percentages between the vertically (4.05) and performance, even though it may be only slight. horizontally (4.04) oriented expansion prints. The 


\section{Print set 10}

\section{Goals}

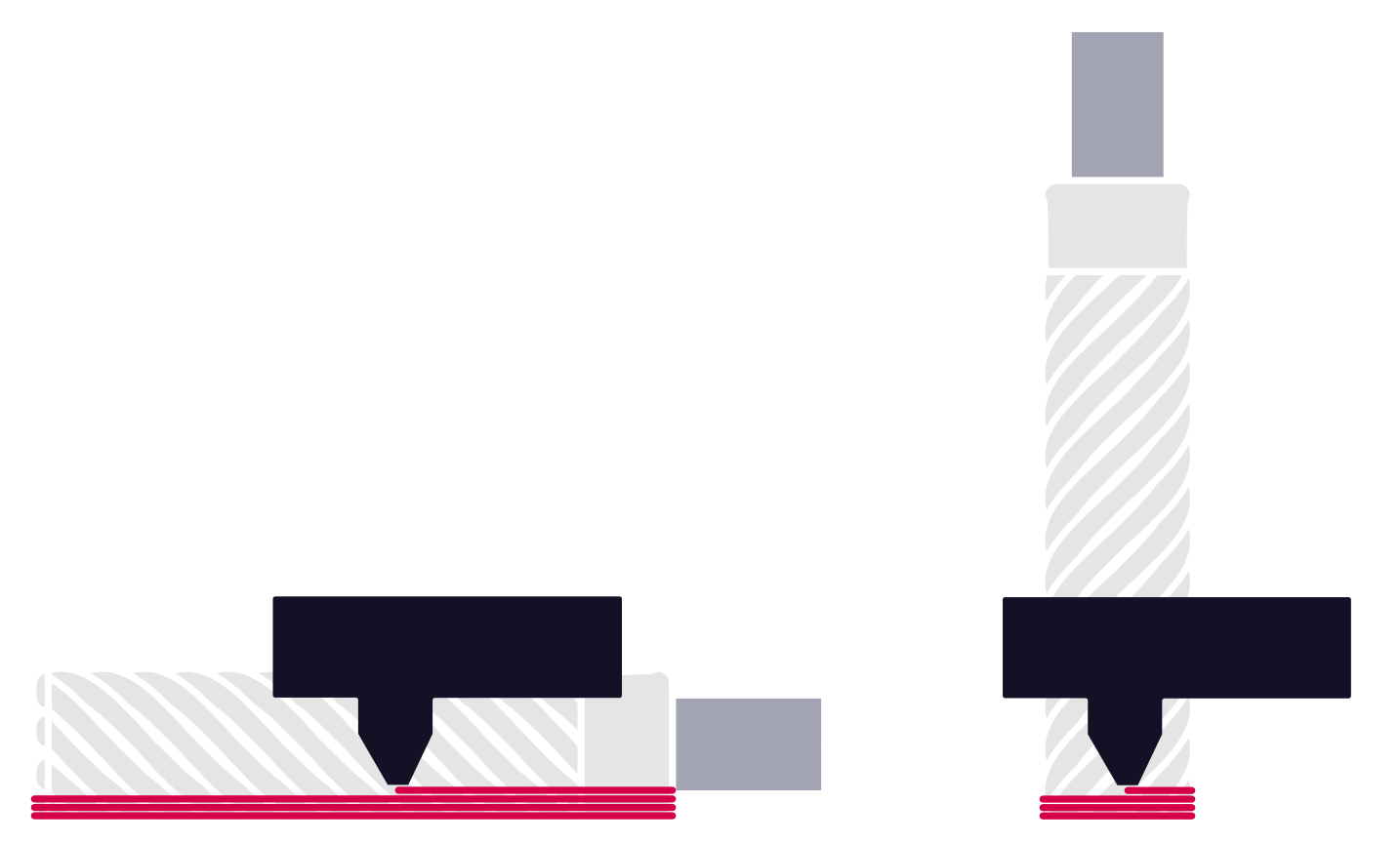

This print set aimed to explore how the UV light power of the Stratasys J750 affects the

performance of the models. The models for this

print set were printed with the lowest UV power,

with the hope that this would allow the resin to fuse

the layers together more than the standard UV light

power that has been used for all other prints. Like

print set 9 , the twisting and expansion actuators

were printed in both horizontal and vertical print

orientations which allowed for direct comparisons

in performance

performance to be made with print set 9 , which
were all printed with standard UV levels.

Figure 76. Horizontal and vertical print orientations. 

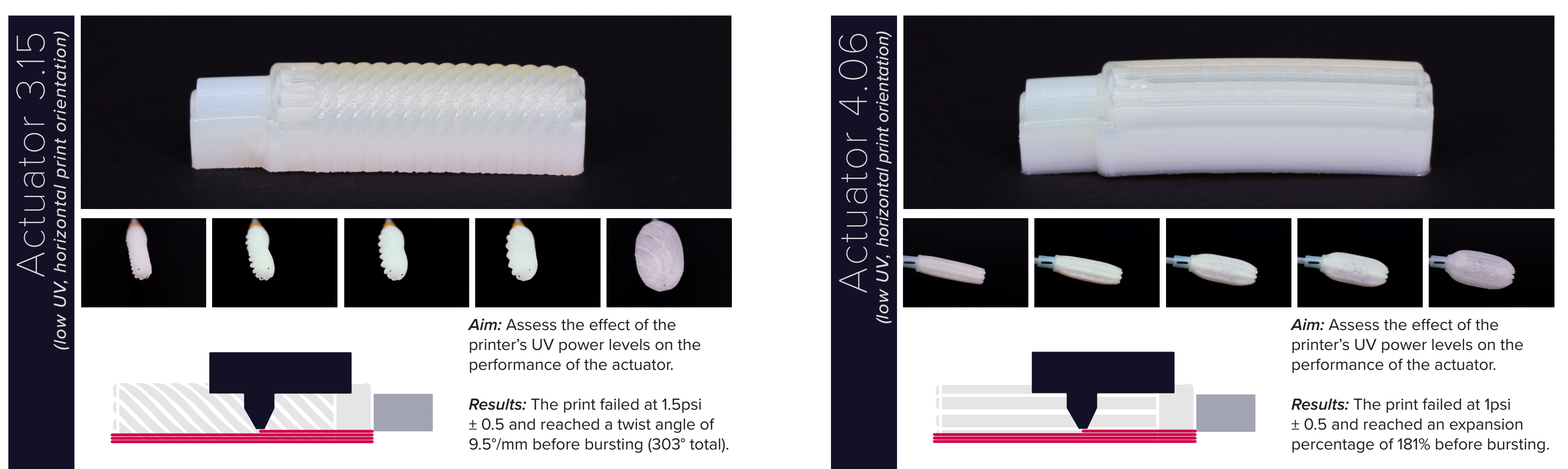

Figure 77 - 77 g. Actuator 3.15 geometry and inflation sequence.
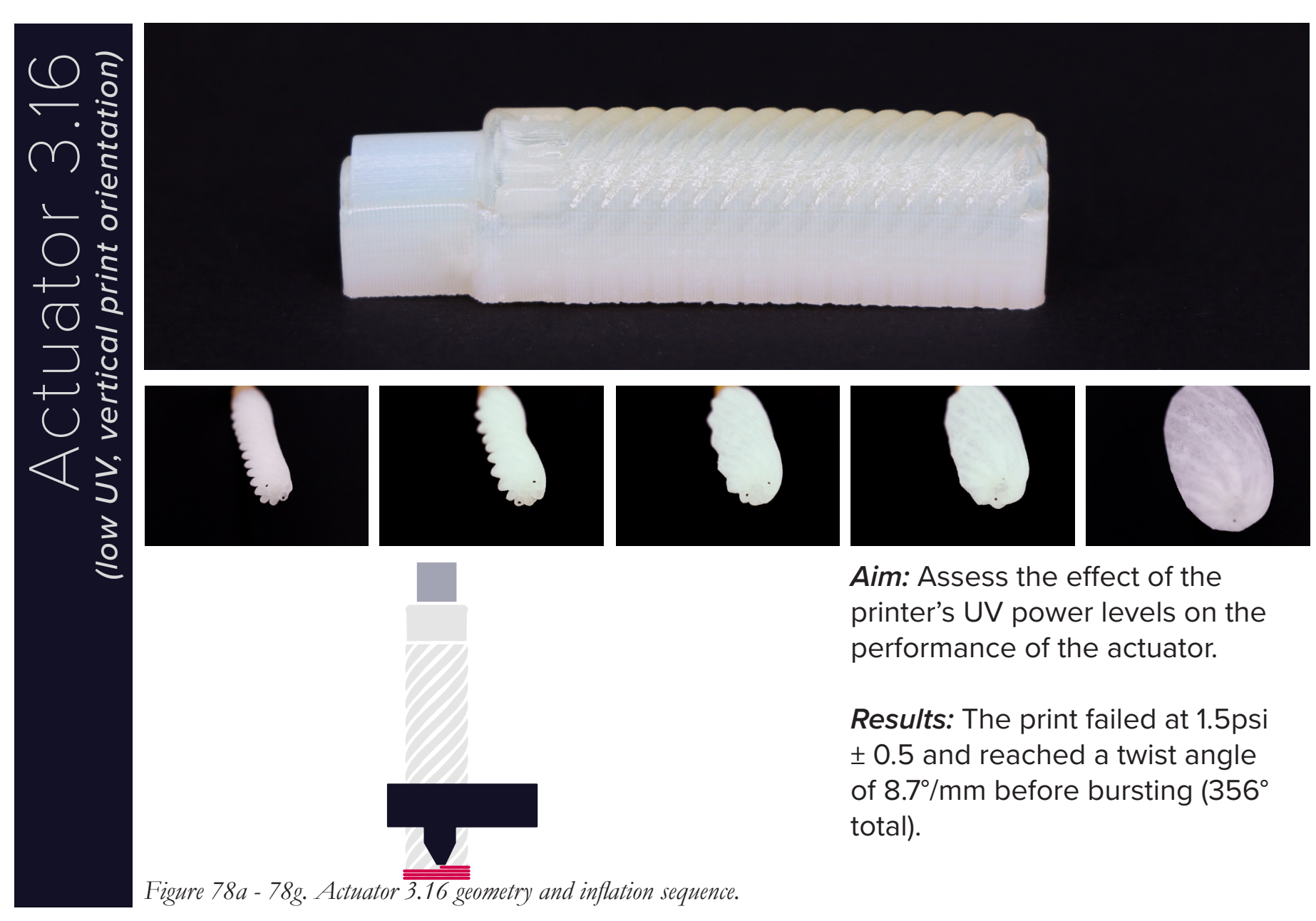

Results: The print failed at $1.5 \mathrm{ps}$

\pm 0.5 and reached a twist angle

of $8.7^{\circ} / \mathrm{mm}$ before bursting $\left(356^{\circ}\right.$

total).

Figure 79a - 79g. Actuator 4.06 geometry and inflation sequence.

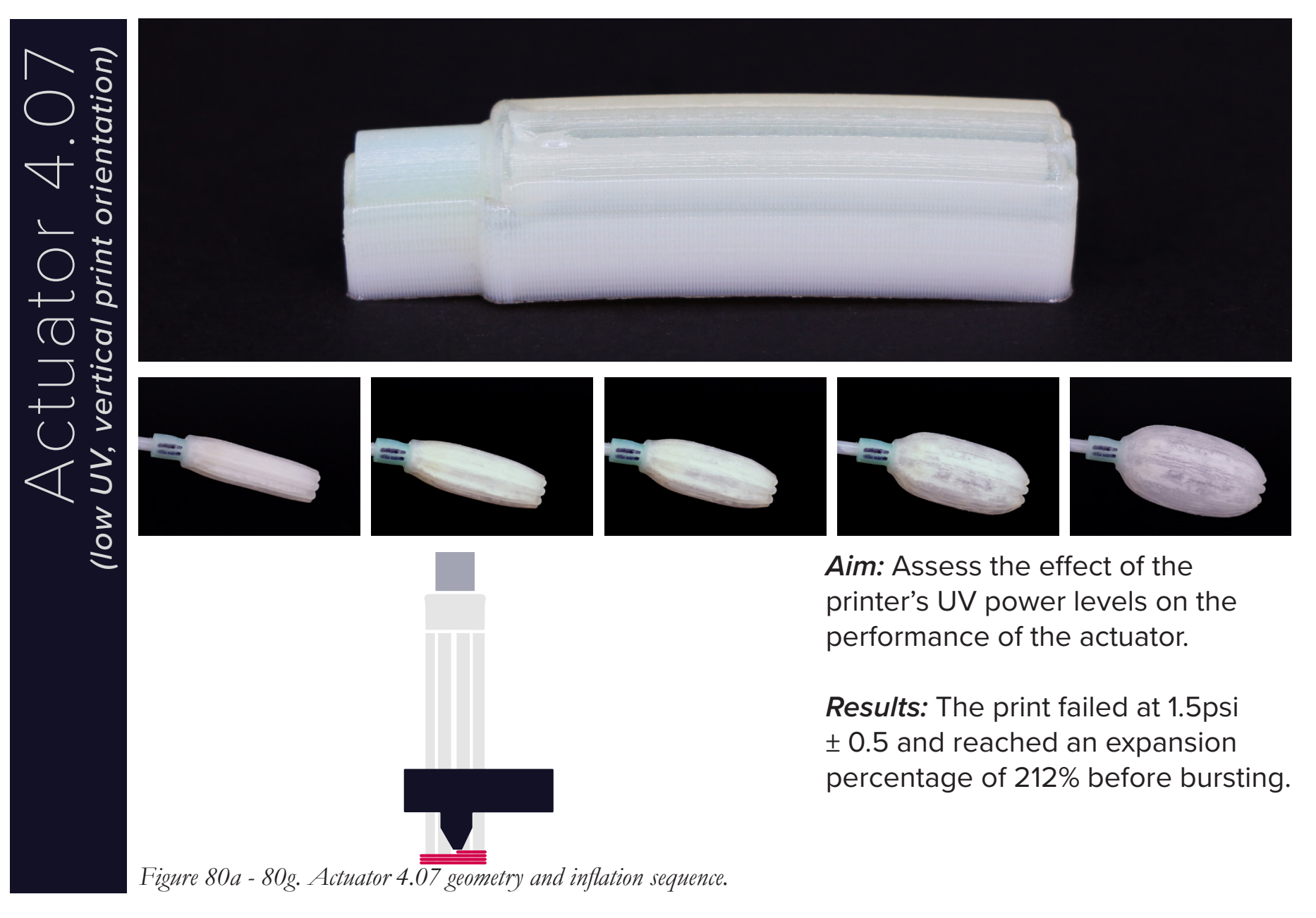




\section{Analysis}

The difference in performance between the two tests is contradictory, as the low UV horizontal expansion test (4.06) outperformed the norma UV horizontal expansion test (4.04) but both low UV twisting tests (315 \& 316) performed worse UV twisting ext (3.15 \& 3.16) peformed worse than the noreal UV (3.12 \& 3.13). This suggests that uncontriled variables such as variations in print quality and manual cleaning are what is changing the performance - not the UV levels. This hypothesis could be tested by printing many of the same model and testing their maximum performances to find averages to determine the

variation in the prints that need to be taken into account. This has not been done for this project as it does not help to accomplish the project goals, and is suggested as a useful topic for future study. 


\section{Print set 11}

\section{Goals}

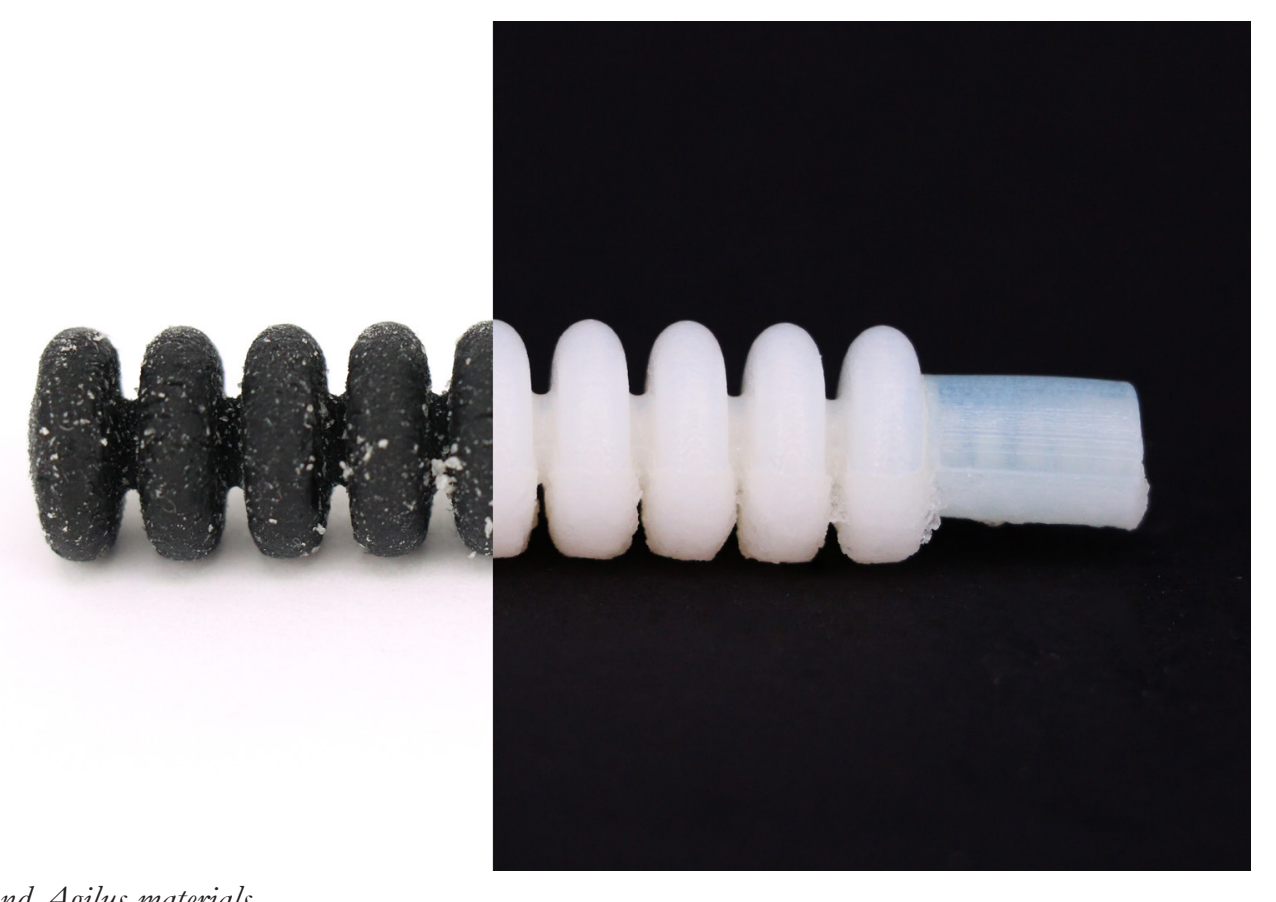

This print set was done on a Connex 350 3D printer rather than the Stratasys $J 750$ so that a different soft material could be trialled. The Connex 350 had Tango, an alternative to Agilus, loaded in its print ray. Tango has slightly different material properties to Agilus, and has a 27SH as opposed to $30 \mathrm{SH}$ for Aglus. This material was tested so that its for Agilus. This material was tested so that its performance could be directly compared with the Agilus material. Each of the main four established primary motions were printed in this material. 


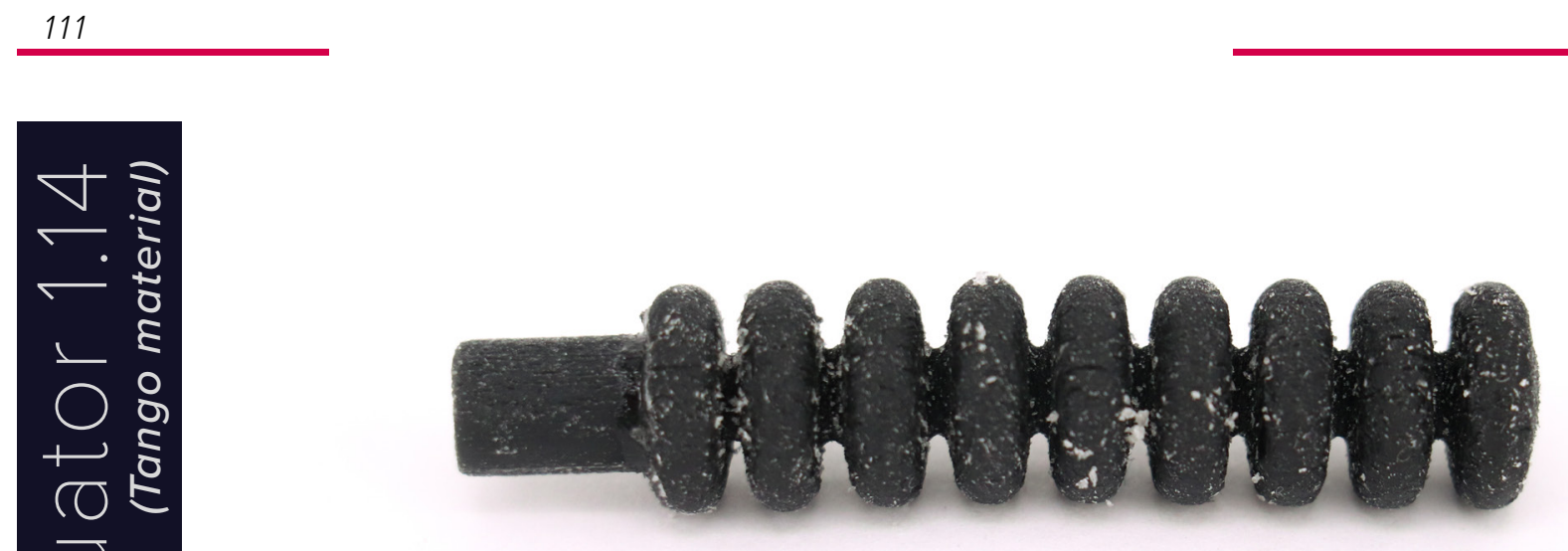

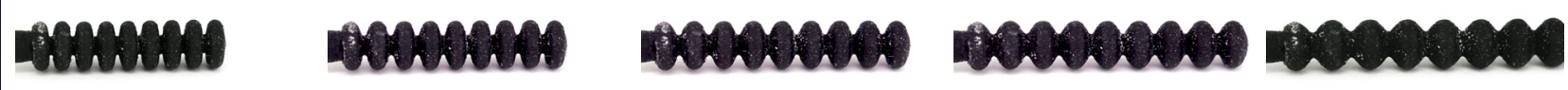

Aim: Assess the performance of the Tango material in comparison to the Agilus material over a number of

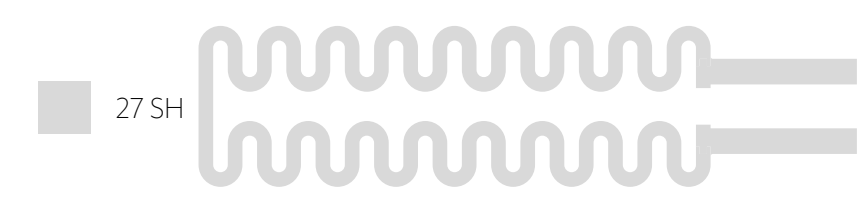
different geometries.

Results: The print failed at 10psi and reached an extension percentage of $166 \%$ before bursting.

Figure 82a - 82g. Actuator 1.14 geometry and inflation sequence.
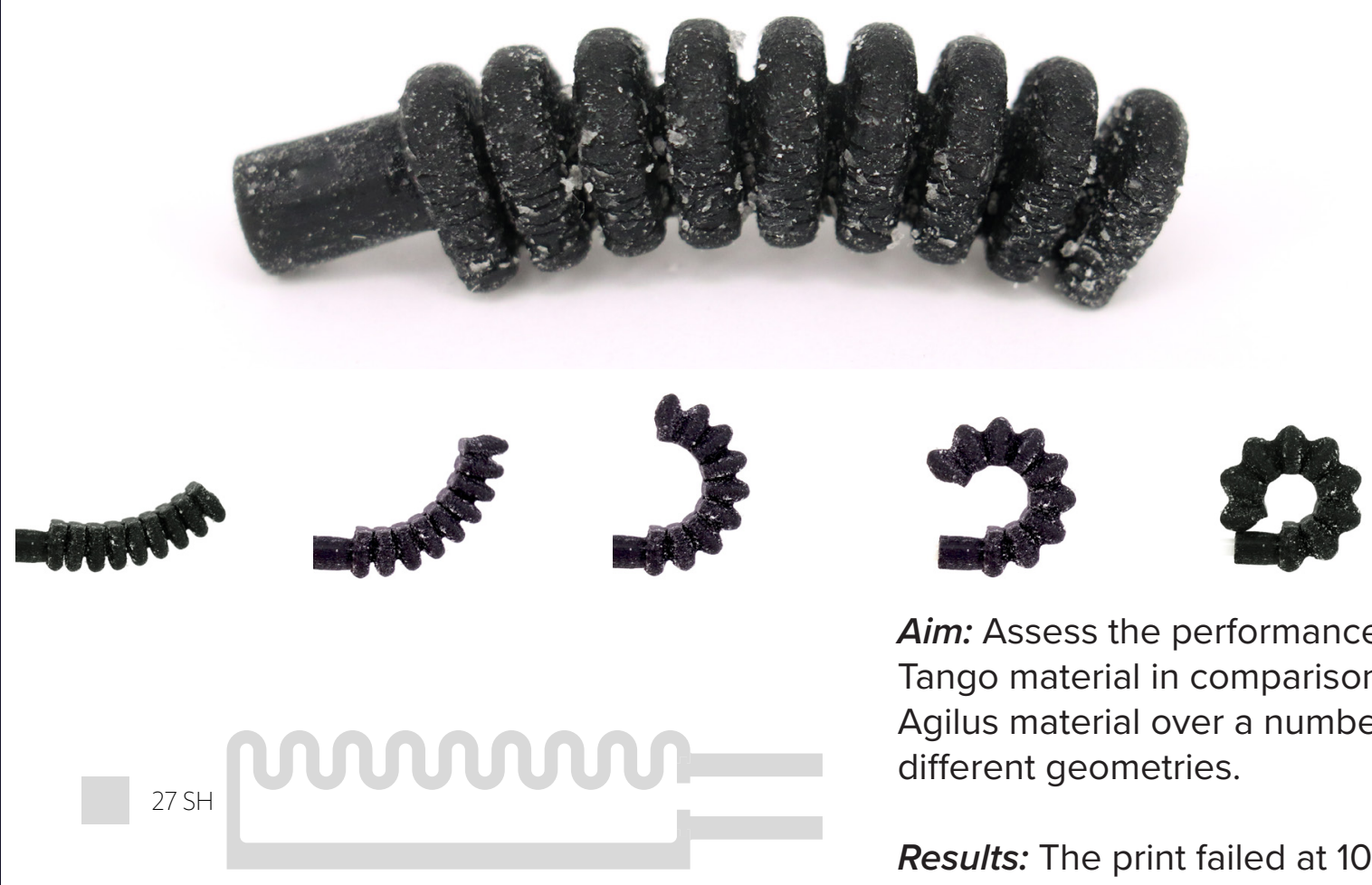

Aim: Assess the performance of the Tango material in comparison to the Agilus material over a number of different geometries.

Results: The print failed at 10psi \pm 0.5 and reached a maximum bend angle of $7.0 \% \mathrm{~mm}$ before bursting (246 total)
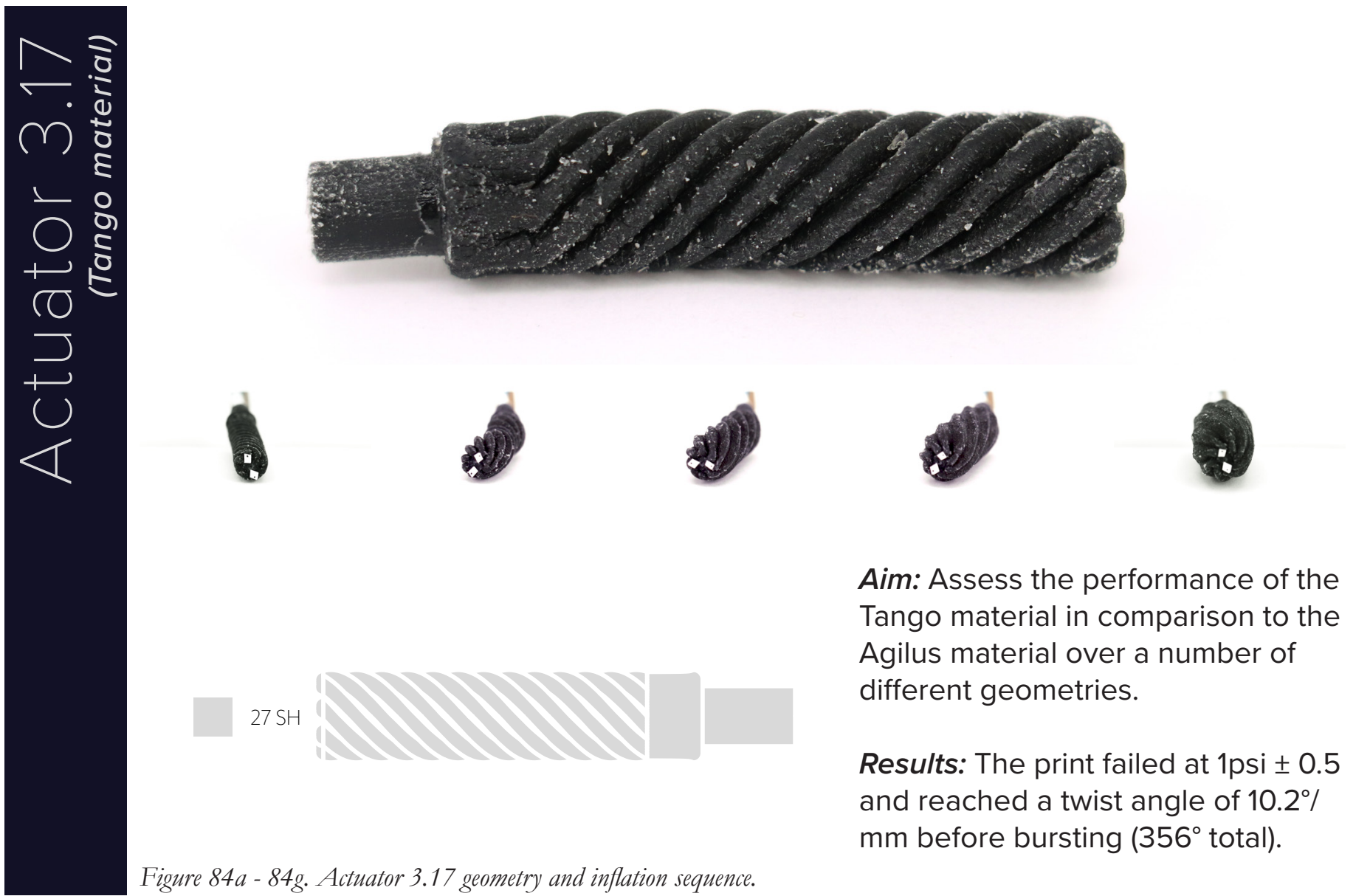

Figure 84a - 84g. Actuator 3.17 geometry and inflation sequence.
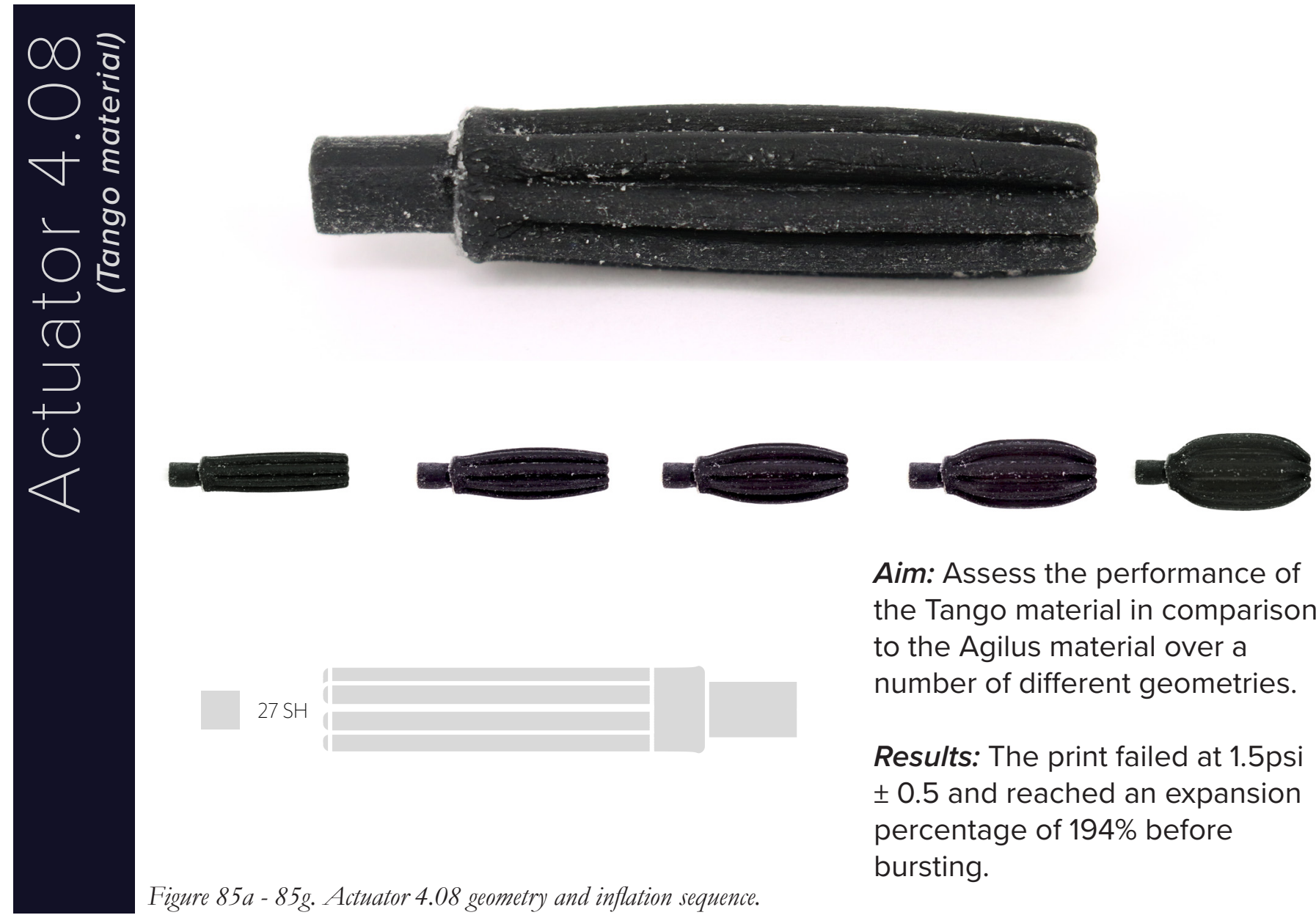


\section{Analysis}

The variations in performance to the Tango prints cannot be made due to the difference in material and their Agilus counterparts were minimal shore hardness, the results for all prints did not The Tango prints for the twisting and extension yield largely different results worth pursuing further geometries achieved larger deformations than their to achieve complex motion.

Agilus counterparts, however, the difference

Agilus counterparts, however, the difference in

performance was it is important to note that although the failure

to $10.0 \% \mathrm{~mm}$ for the twisting print, $166 \%$ compared pressure of actuators 1.14 and 2.08 were 10 psi, they

to $162 \%$ for the extension print). The expansion and deformed to reach their actuated state at a much

bending geometries performed worse than their lower pressure. This large difference in failure Agilus counterparts; $194 \%$ compared to $211 \%$ for pressure was indicative of the issues that arose the expansion geometry, and $7.2 \% \mathrm{~mm}$ compared around the complex actuators throughout the

to $7.0 \% \mathrm{~mm}$ for the bending geometry. Although a experimentation.

direct comparison in the performance of materials 


\section{Print set 12}

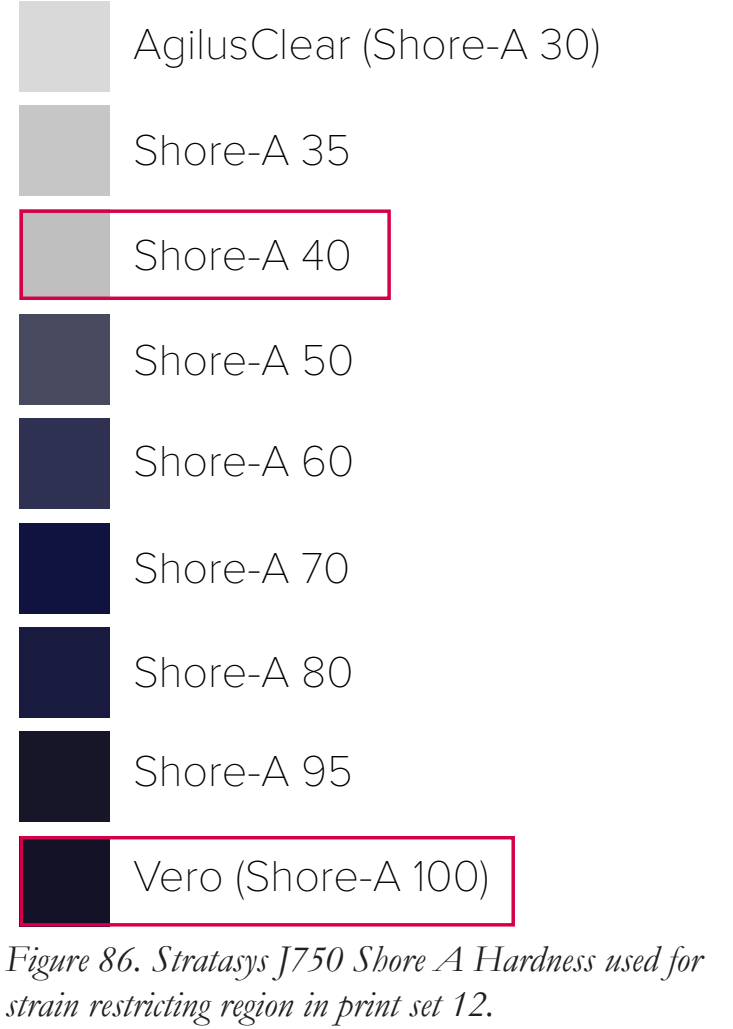

\section{Goals}

This print set aimed to explore how the shore hardness of the strain restricting layer in the bending actuator would affect the total bend angle (Figure 86). This was done to further understand the relationship between materiality and geometry. 

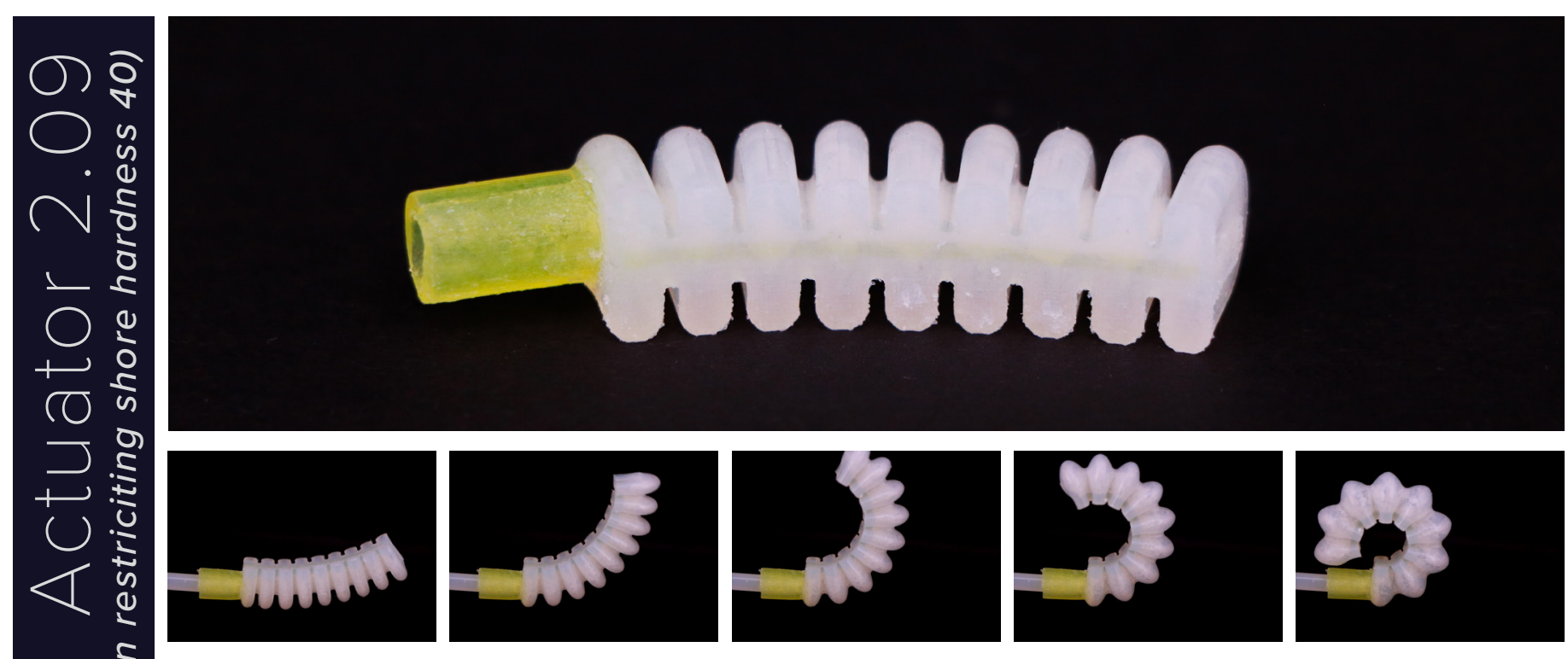

Aim: Assess the effect of the shore

hardness of the strain restricting

- $40 \mathrm{SH}$ 月 2 region on the total deformation.

Results: The print was able to reach a maximum bend angle of $7.1 \% \mathrm{~mm}$

This print set shows that the shore hardness of the strain restricting region on the bending actuator cres have an effect on its performance. The low shove hardness print (2.09) achmance. The low shore hardness print (2.09) achieved a very similar total bend to actuator $2.04(7.1 \% \mathrm{~mm}$ to $7.2 \%$ $\mathrm{mm})$. From this it can be inferred that, if the shore hardness is too high, this will start to decrease the performance of the actuator, otherwise its effect is negligible.

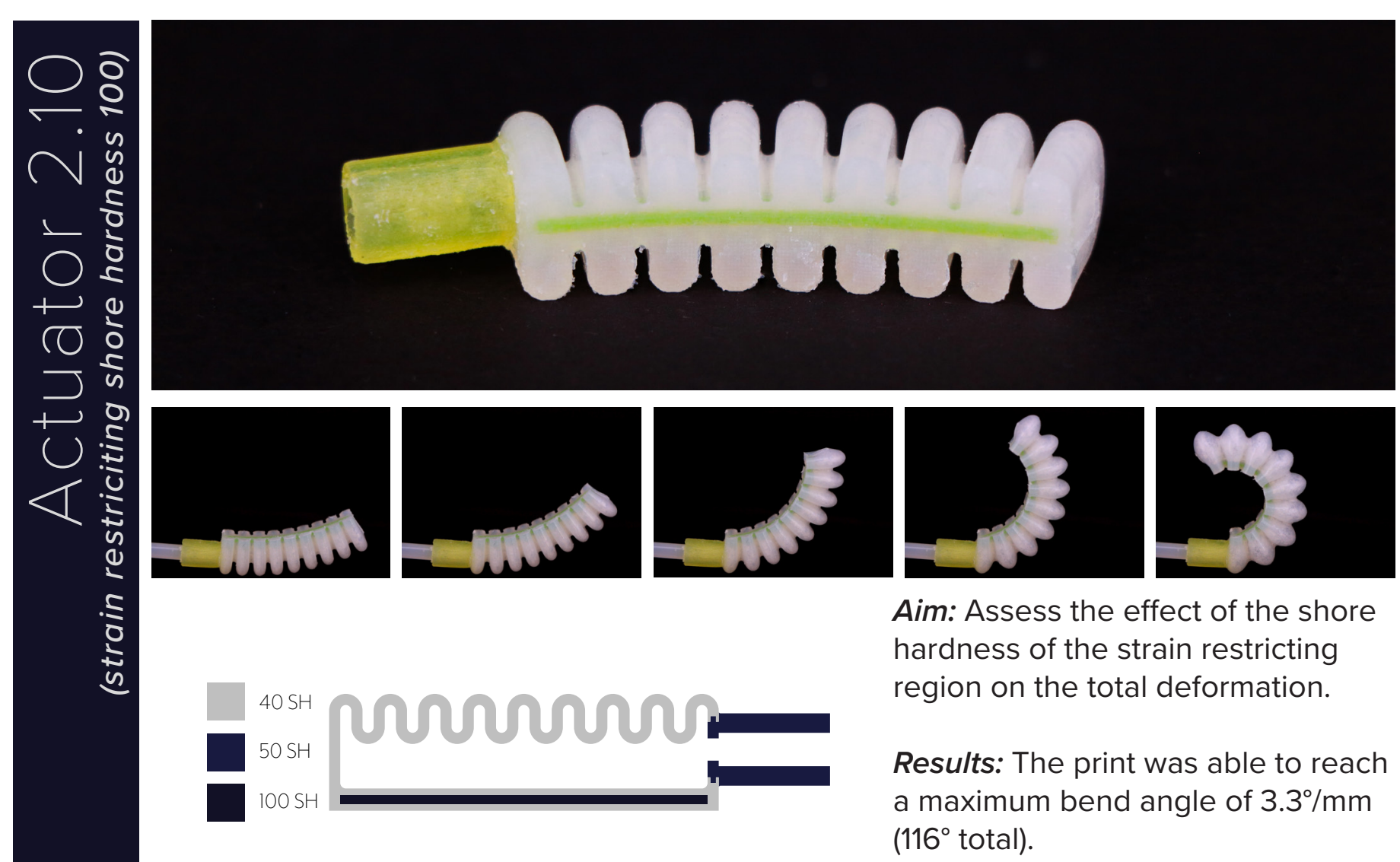


FINAL PRINTS 


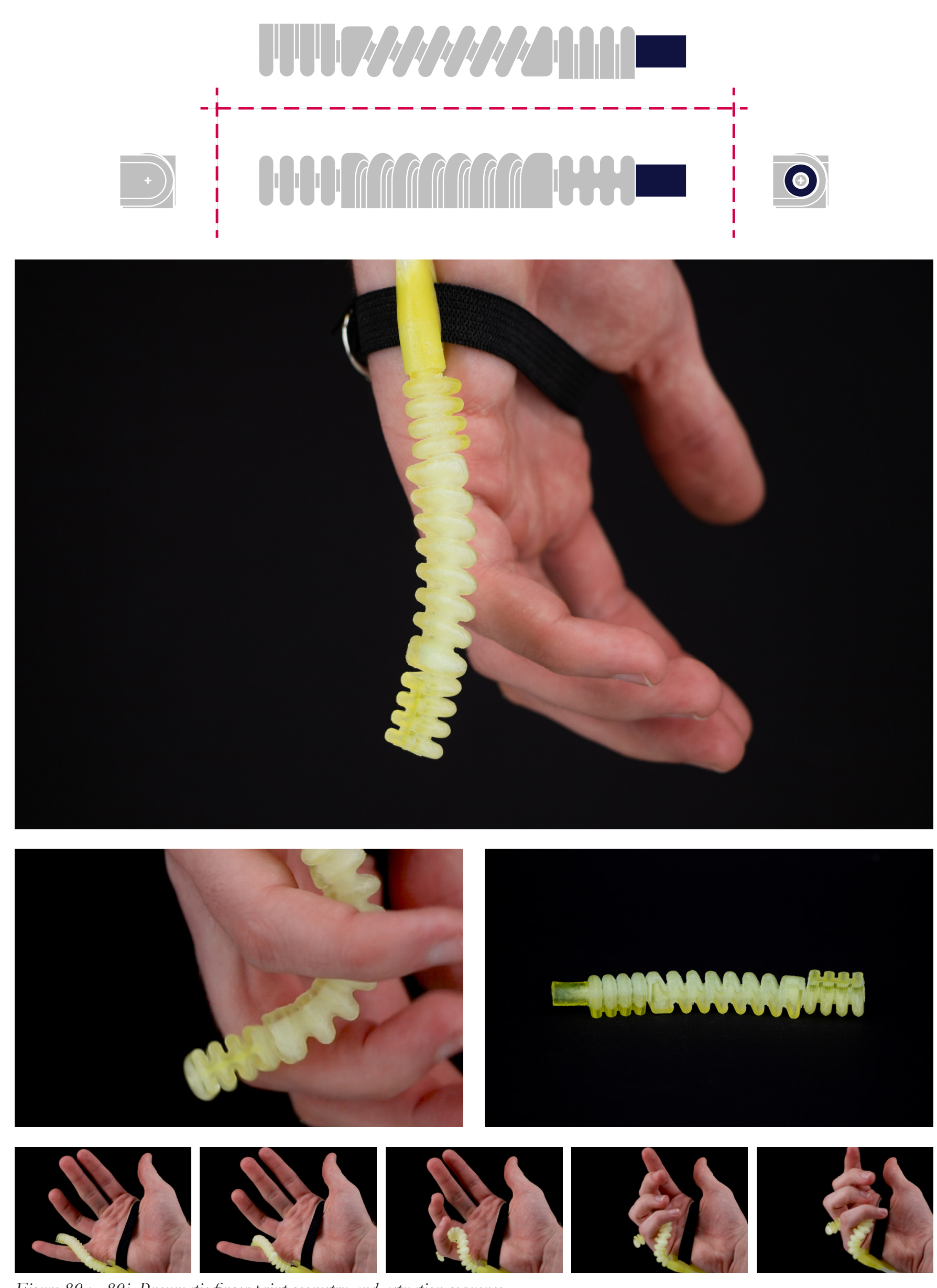

\section{Pneumatic finger}

Taking inspiration from prosthetic artist and researcher Dani Clode and her Third Thumb project (2017), this print demonstrates an actuation with multiple segments capable of three-dimensional motion. By combing different orientations of the bending geometry as well as the bending/ twisting geometry, the print is able to wrap around the pinkie finger when inflated. Although this print demonstrates no practical purpose in itself, it demonstrates how configuring the motions that have been identified throughout the literature can be utilised to achieve complex actuation capable of human interaction. 


\section{Gripper}

This final gripper modified the bending motion to have a pointed tip and connected three actuators to a printed pneumatic splitter. The geometry was modified so that it could pick up a larger range of objects as the pointed tip allowed it to curl further around objects. This print successfully demonstrates the compliance of soft robots, as the gripper was able to pick up a lemon, half a garlic clove, a cherry tomato, and a marble all without any trouble. The gripper changed its hold depending on the size of the object it was wrapping around, and as a result, was able to pick up objects of varying size and weight.
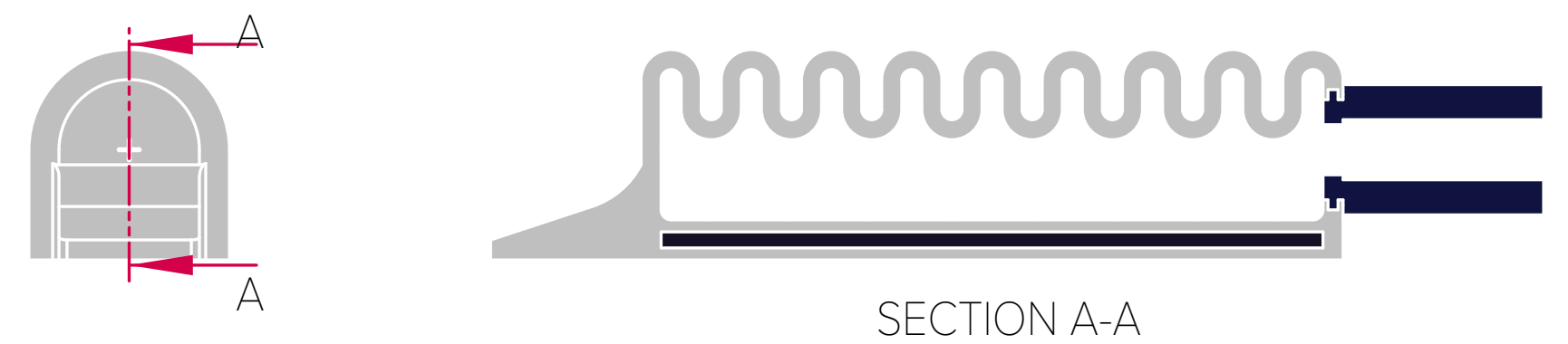

SECTION A-A
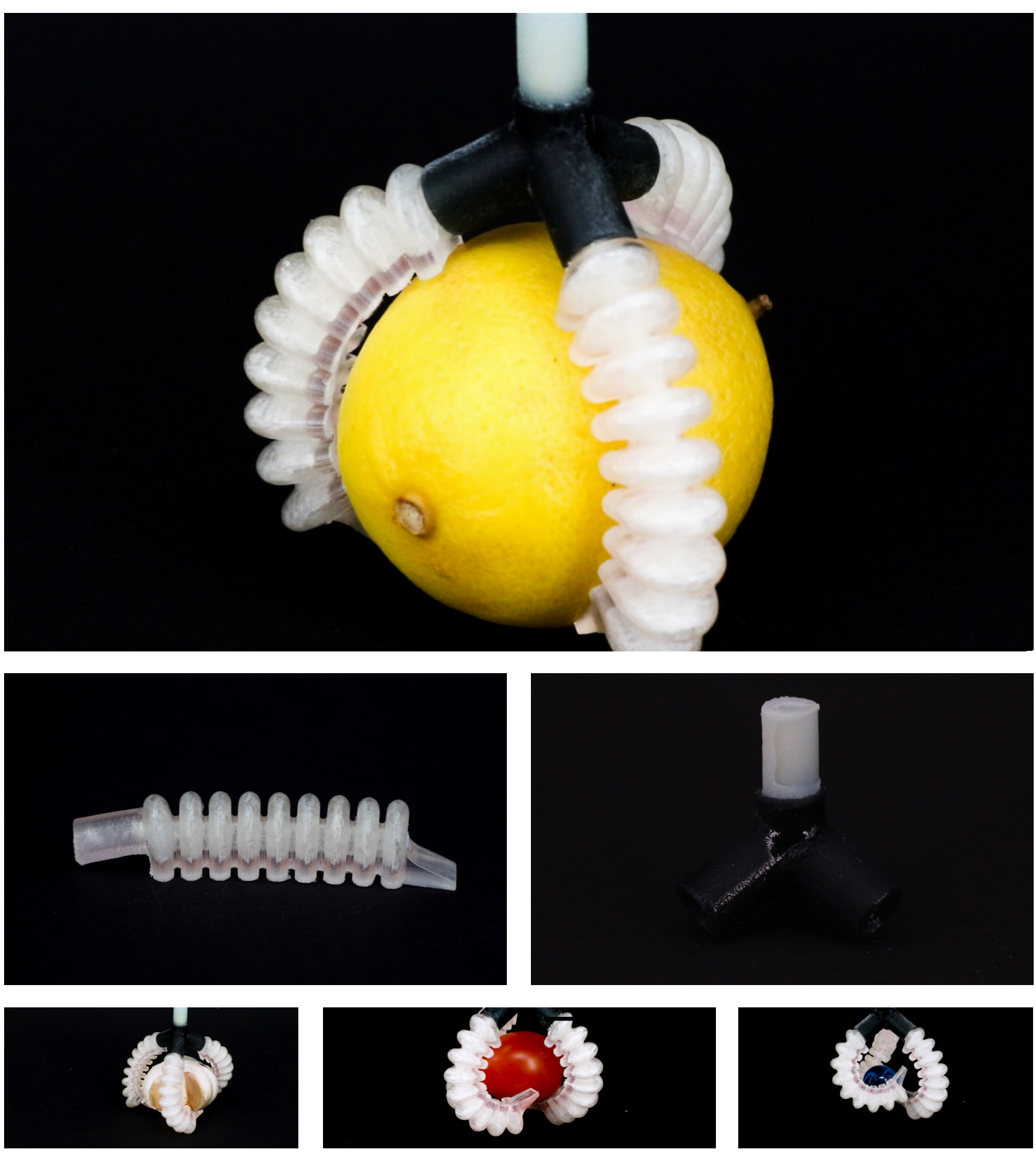

Figure 90a - 90g. Gripper print geometry and demonstration gripping various objecs. 

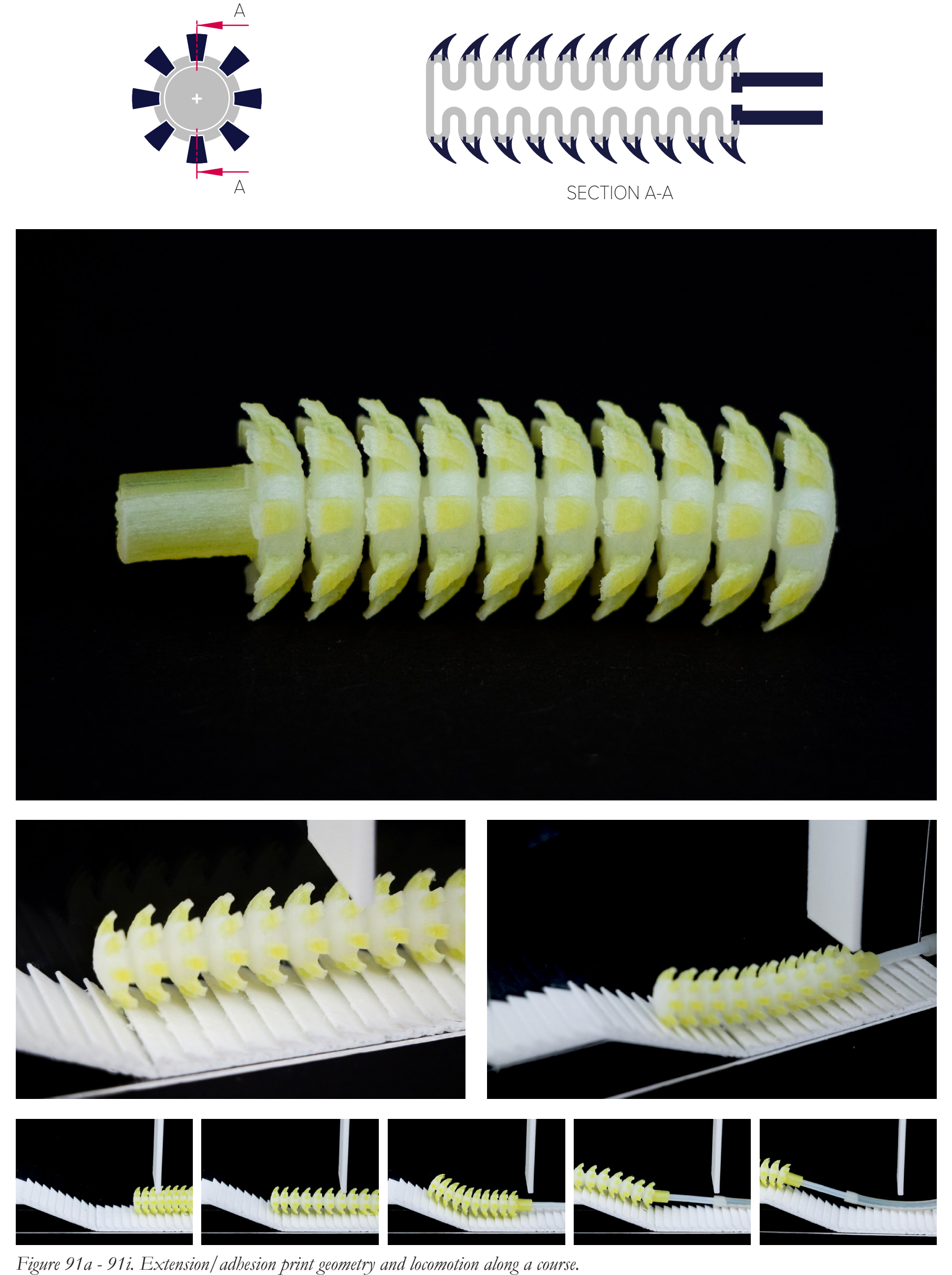

Locomotive extender

By utilising the previous success of the actuator 1.11, this print utilises the deformation achieved by the extension motion and the added gripping abilities of mechanical interlocking geometry to traverse a short obstacle course. The actuator was able to crawl through a small gap, traverse a length of level terrain, and scale a small incline by switching between inflation and deflation states. This print, although reliant on a special interlocking terrain, demonstrates the locomotive capabilities of a multi-material 3D printed soft robot actuated through only one pneumatic input. 
DISCUSSION 


\section{Summary}

This portfolio has explored the possibilities and complexity of motion achievable in pneumatically actuated soft robots. By taking inspiration from muscular hydrostats found across the animal kingdom, 3 primary motions were identified, explored, optimised, and finally combined to achieve a variety of complex motions.

This research has demonstrated that soft robots printed on the Stratasys J750 can not only actuate with performance that can rival other soft robots using alternative fabrication methods, but it also gives a further insight into the relationship between geometry and materiality during actuation. This is most evident in the bending motion. As the most documented soft robotic motion, this research has demonstrated that bending is also very achievable through multi-material printing. By experimenting with the shore hardness of the strain restricting layer in the actuator it was found that materiality can have a substantial impact on the performance of the actuator. It was also found that the geometry of the actuator significantly impacts the performance - possibly more so than the materiality as seen in actuators 2.03 and 2.04 (Figure 92). Although

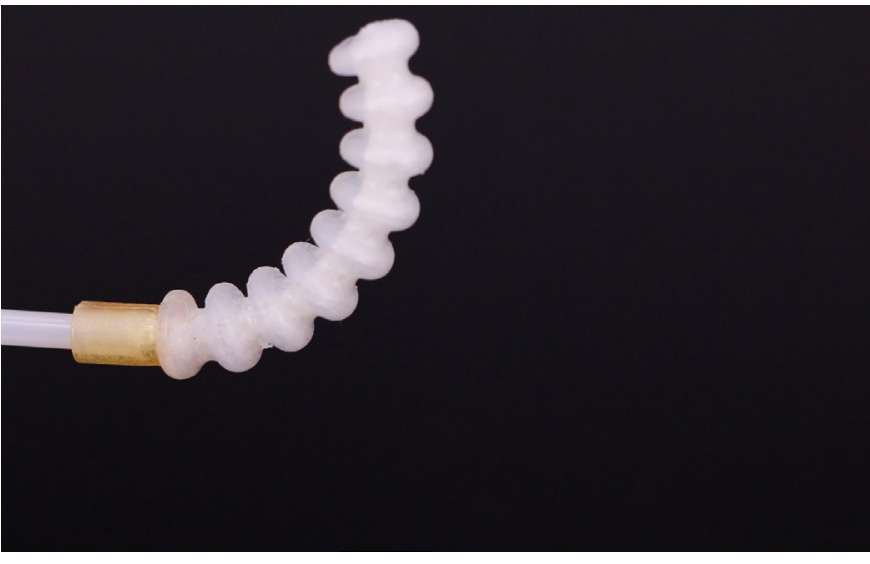

these actuators are not directly comparable, they are derived from the same geometry and demonstrate that in this research, it was muc easier to achieve the desired motion through altering the geometry of the actuator rather than the materiality.

The motion that required the fewest changes to its geometry was the extension actuato This was the motion that served as a base model for all other motions, and the geometry was based on literary examples (Drotman et al., 2017, p.5533) which allowed for early success. As this motion proved to be successful and reliable, it was used to test new design ideas. The extension geometry was used as a base geometry for the internal valve concepts and the scaled print. The internal valves were ultimately unsuccessful, as they did not reliably restrict airflow to sections of the actuato The scaled prints proved that when the wal thickness of the actuators was increased, they were less prone to material tears and bursting during inflation. Unfortunately, due to the large cost of the larger scale prints, more widespread large scale testing was not feasible for this portfolio.

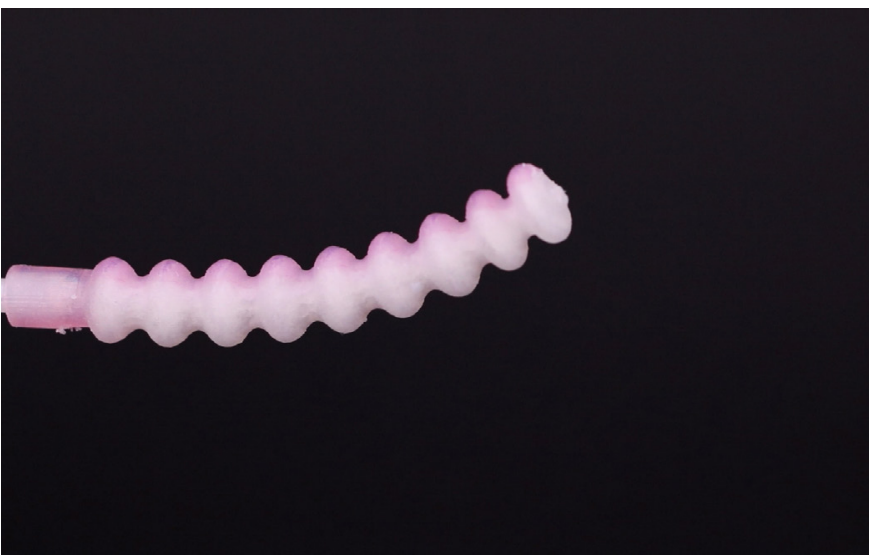

The motion that yielded the most success was the twisting geometry. Once the bellows were patterned axially (first in print set 3 ) the twisting actuators achieved much larger twist angles than their literary counterparts. The largest twist angle found in an actuator in the literature was $5.6 \% \mathrm{~mm}$ (Schaffner et al., 2018) which utilised helically arranged strain restricting regions to achieve twist. This research presents a maximum twist angle of $12.4 \% \mathrm{~mm}$ (actuator 3.07 ) although the actuation with this geometry was unreliable and material failures occurred after repeated actuation. A twist angle of $9.7 \% \mathrm{~mm}$ (actuator 3.08) was reached for which the actuator was able to reliably reach across repeated tests (Figure 93). This result is significant because it demonstrates a novel geometry for twisting soft robots that can achieve a high twist angle that can't be fabricated with a moulding and casting process. As an extension of the twisting geometry, an expansion motion was also created which yielded some success. This

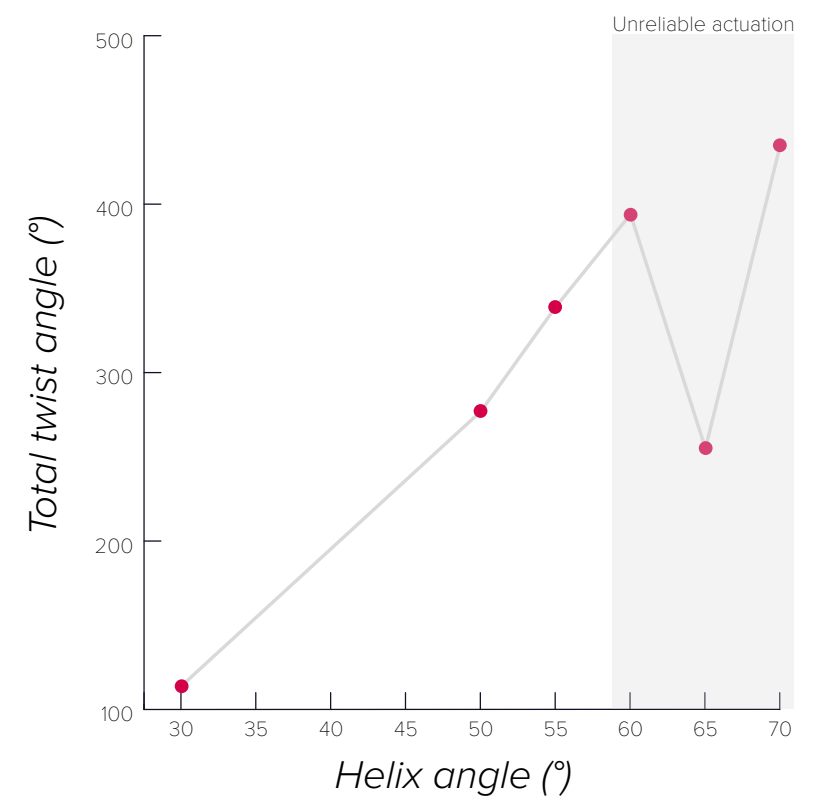

Figure 93. Impact of the helix angle of the twisting geometry on the total twist angle. motion was achieved when the helix angle of the twisting Solidworks model was set to $0^{\circ}$. This allows for a complex model with this motion to easily switch between the twisting and expansion geometry without having to switch separate models in and out of the assembly.

The early testing of adhesion geometries demonstrated that mechanical interlocking was the adhesion method most successful at the printable resolution of the Stratasys 1750 . All of the geometries which allowed for mechanical interlocking significantly outperformed the geometries which did not. This was particularly evident on the felt material, which worked much like Velcro to allow for the mechanica interlocking geometries to stay attached when upside down. This was expanded upon for the final locomotive extender concept, which successfully utilised mechanical interlocking surface adhesion to achieve linear motion a $2.3 \mathrm{~mm} / \mathrm{s}$.

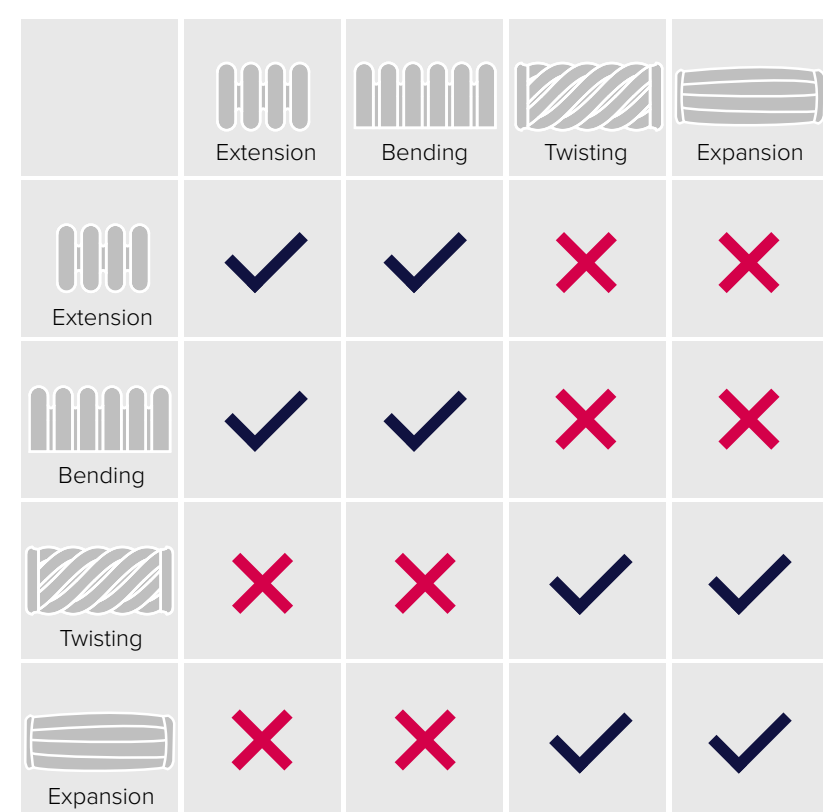

Figure 94. Successful combinations of motion for complex actuation. 


\section{Limitations}

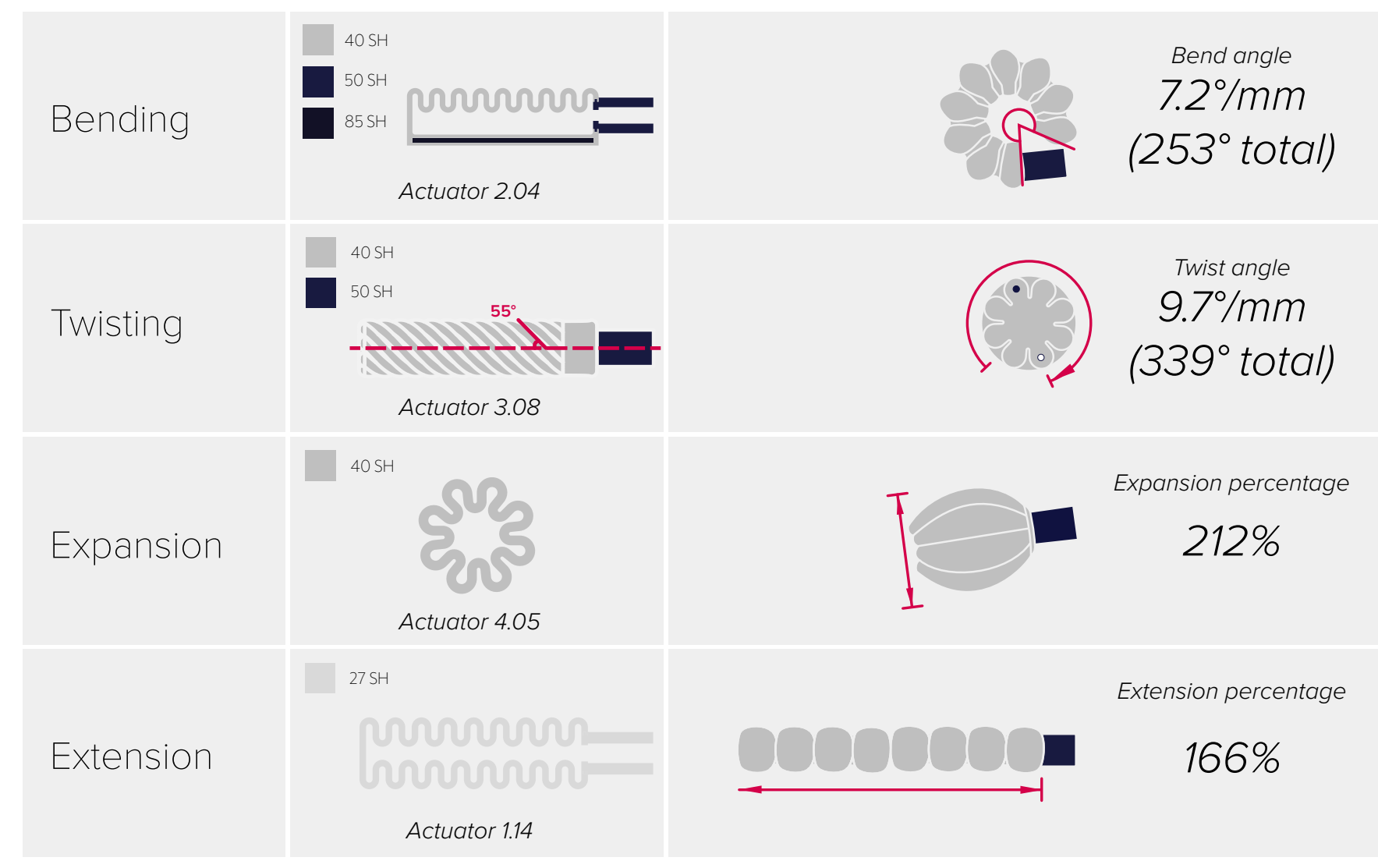

The main drawback that was encountered during this research was that the soft Agilus material on the Stratasys $\mathrm{J} 750$ was prone to tearing, especially after repeated actuation (Figure 96). Experiments were done to assess how the print orientation of these models and UV light print exposure would affect their performance, as well as how the Tango material compares both in terms of tearing and overall performance of motion. The only one of these variables that did have an impact on the performance of the actuators was the print orientation, although for this research it was minimally significant.

The tearing of the material also had a large impact on the possibilities for complex actuation. All prints that included both the extension/bending geometry as well as the twisting/expansion geometry failed to some extent (Figure 94). This was because the twisting/expansion geometry actuator burst at a lower pressure (1.5psi) than the actuation pressure of the extension/bending geometry actuator (approx. 4psi). This was particularly evident in Print set 11. This was largely caused by the different base geometries of the models - the extension and the bending actuators were derived from the same base geometry, as were the twisting and expansion actuators. Further changes to parameters such as wall thickness and shore hardness could be made for more successful results, however, this was not tested as it would require drastic alterations to be made to the base geometry of the actuators.

This research aimed to assess the possibilities of soft robotic actuation through one pneumatic input, and limitations were reached pneumatic input, and limitations were reached
as the edge of the range of capabilities using this method was encountered. Sequencing of motion proved to be challenging using one input because the internal valves were unsuccessful. Although they were able to restrict airflow between sections for the initial inflation of the actuator, this was not able to be repeated. This was likely because the initial restriction of airflow was caused by an internal blockage of support material or that the airflow passing through the valve damaged the valve so that it was unable to restrict airflow again This suggests that sequencing of motion may require the utilisation of multiple pneumatic inputs.

Limited access to the Stratasys $\mathrm{J} 750$ printer due to Covid-19 impacted the number of prints that were possible in the experimentation section of this research. Earthquake strengthening of the building also took place during the experimentation phase of this portfolio, which further restricted the access to the Stratasys J750 printer. In response, compromises were made regarding the total number of print sets that could be produced, and the range of goals for the print sets was lowered. As experiments had to be condensed onto fewer print trays, the learning opportunities and iterative changes that could be made between each print set was restricted.

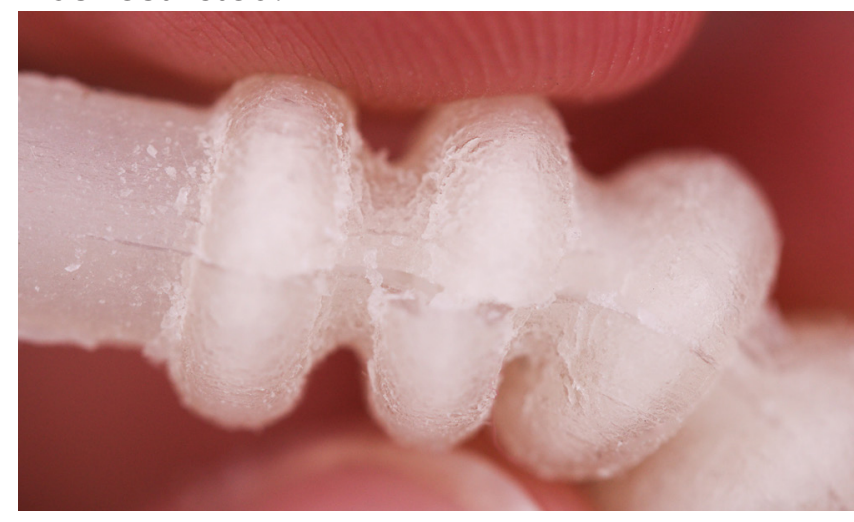

Figure 96. Print set 1 material tear 


\section{Future Research}

This portfolio demonstrates an exploration of Polyjet printed soft robots with a singular pneumatic input. As an extension of this, future research could explore Polyjet printing of soft robots with multiple pneumatic channels to expand on the control and capabilities of the actuators. As was found across the literature, soft robots with multiple channels can allow for individual control of certain sections for complex actuators, which can achieve advanced motion. This portfolio has encompassed a wide range of experiments through one pneumatic channel, and in doing so, the limits of control that are possible using this method were found. Future research should take this into account while expanding on the overall possibilities of motion with additional pneumatic channels.

Future research is needed to establish the performance of complex Polyjet printed soft robots at a larger scale. Due to the large cost

of large scale prints, these were explored very little in this portfolio, however, they offered promise in their strength and actuation repeatability - shown especially in actuato 1.10 in print set 7 . This could allow for further are not in pressures.

Simulation software was used for this research to create a digital model to compare to the experimental results. Solidworks, Abaqus, and ANSYS were all tested to try to simulate pneumatic actuation but none achieved results comparable to the experimental data. If good simulation results could be achieved this could be used as a tool to accurately showcase how a parametric model system will inflate before it was printed, which would mean far fewer experiments would be needed before the intended result was accomplished.

\section{Implications}

The method used to parametrically build CAD models of the actuators provides new insight into the role of software to inform soft robots. By using equations to drive the key dimensions and parameters of Solidworks models, a streamlined design process has been created which allow for dexterity in the fine control, modification, and combination of the models. This was utilised to efficiently design each print, so that more time could be spent on testing and creation of new prints. As demonstrated in the final pneumatic finger design, the flexibility of motion shown in this research can allow for potential design opportunities which prioritise the aesthetic qualities of motion rather than the functional qualities. By applying this research method to future design projects, unforeseen aesthetic and emotional opportunities could be uncovered.

This research builds on existing research that shows how, by taking inspiration from biology, designs can be enhanced. Biological inspiration has been used not only to inform how certain

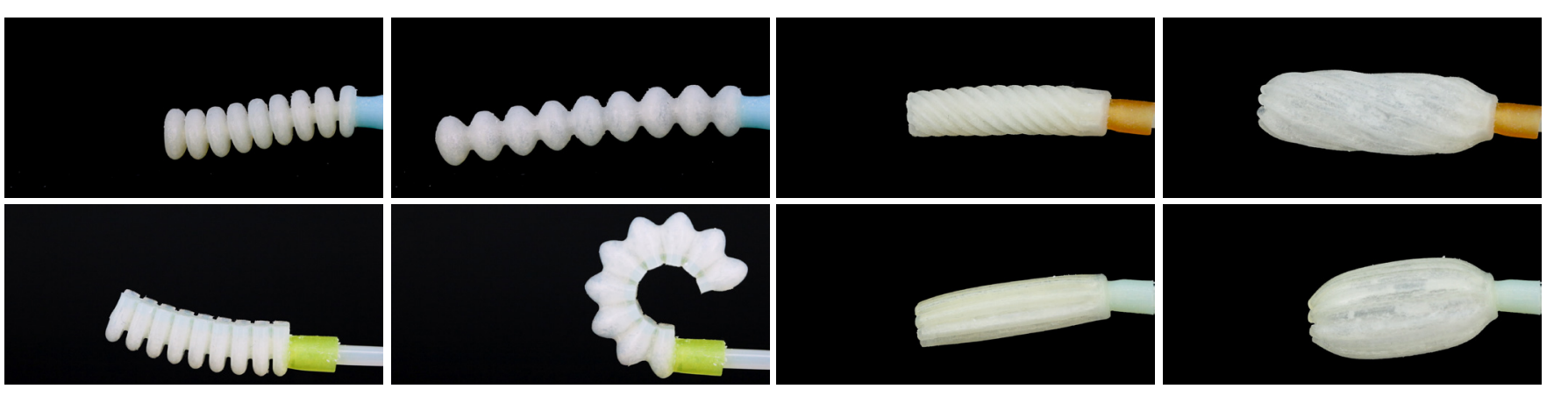

motions function in natural systems and how this can be synthetically replicated, but also in later stages of the design process to increase performance. The final locomotive extender showcases a method for how mechanical interlocking adhesion can be exploited to allow for locomotion in a simple bellows-type actuator (Figure 98)

Very limited research in the field of soft robotics has been done using Polyjet printing. The variety in materiality and geometry in this research vastly expands on the body of knowledge in this field, and because of that, has produced new information regarding the pros and cons on using this technology as a fabrication method for soft robots. The Agilus material created problems concerning tearing and bursting of the actuators, but the geometries for actuators that yielded the mos success could possibly be printed with more success using other 3D printing technologies that allow for geometric freedom similar to the Stratasys $\mathbf{J 7 5 0}$

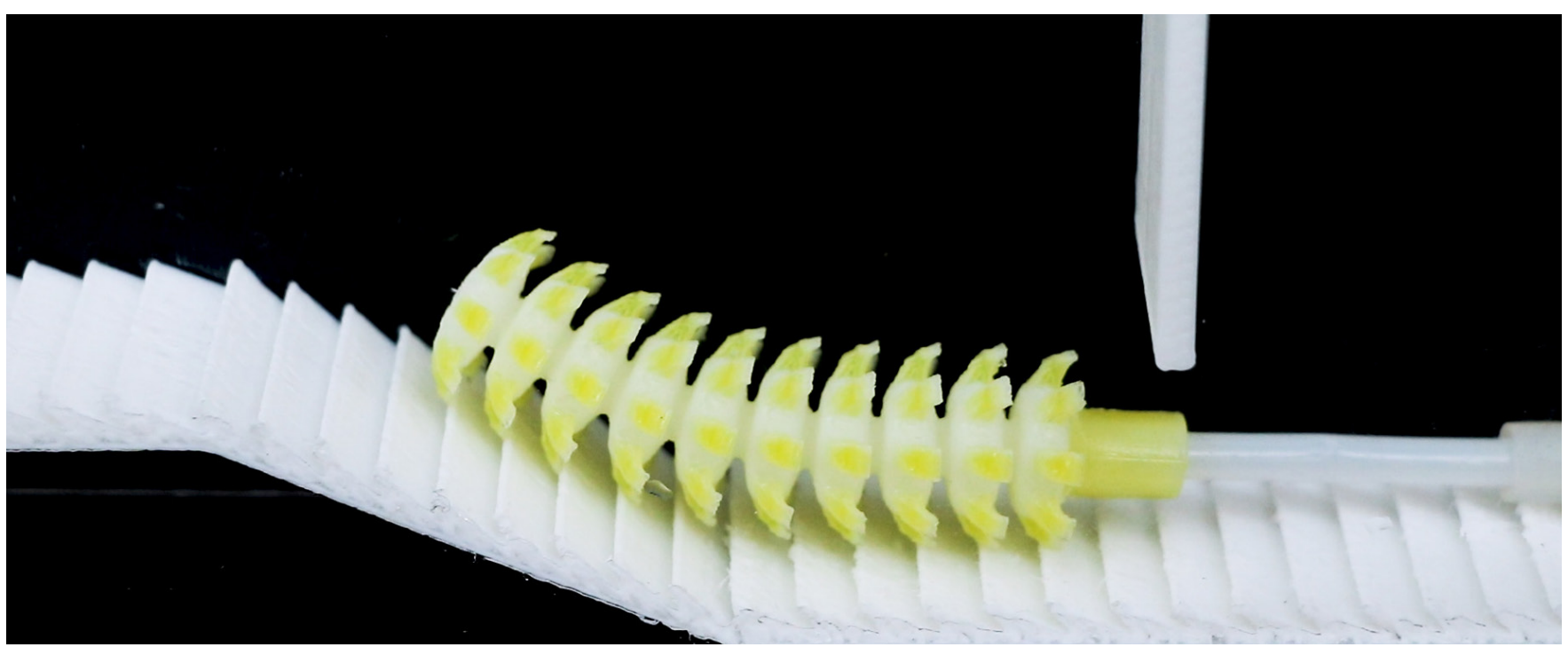




\section{Applications}

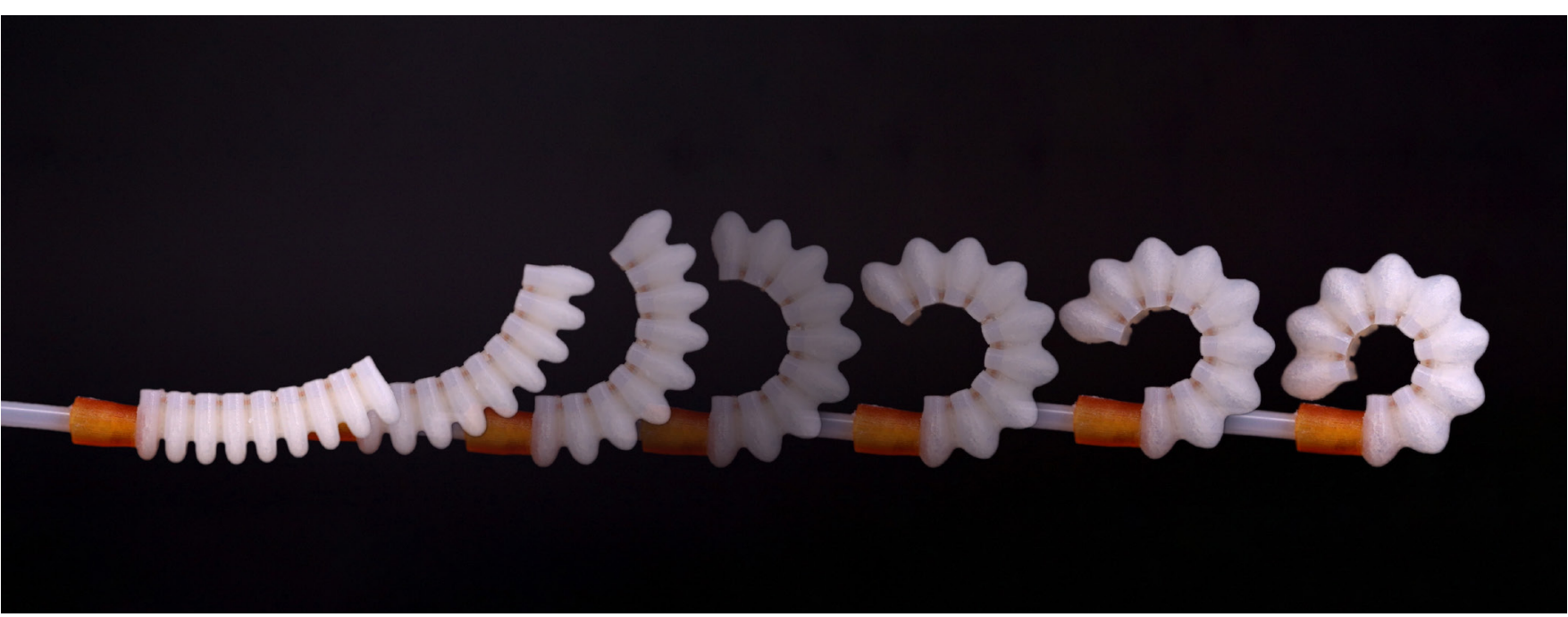

As this research gives a method for combining modular sections of motion, it can be used for a wide range of applications. As shown in the final print set, the parametric models can be modified and configured for vastly different purposes. As identified in the literature review, end effectors for grippers have large commercial potential (MGrip, n.d.). By utilising the fine control of the models, end effectors for a gripper could be configured for different applications, such as produce and baked goods at different scales. These grippers offer a lot of potential as they are able to pick up irregular objects with organic forms. This was shown in this project in the final gripper which was able to pick up objects from marbles to lemons - objects that have a large difference in size and mass.

ocomotion is also an area with large potential for this research. Locomotion has been demonstrated with one pneumatic inpu and adhesion geometry switching between inflation and deflation phases. By attaching a camera, these robots could be used to navigate and assess hazardous terrain such as nuclear disaster sites or to navigate areas that humans can't fit in. Currently, successful locomotion is only achievable on rough surfaces as mechanical interlocking is required although this could be refined through future research.

This research also has potential applications for devices that assist or enhance human ability. Evidence from the literature shows how soft robots can be beneficial for stroke rehabilitation devices for the hand. Although this research doesn't demonstrate specific human-oriented practical applications, the sixth finger final print shows how complex soft robotic motion could be used to enhance the human body. 
CONCLUSION 
This research aimed to explore how multimaterial 3D printing could be used to fabricate singular channel pneumatically actuated soft robots capable of complex actuation for advanced applications. This has been shown through a series of multi-material 3D printed soft robotic actuators which rely on parametric control of software and biological inspiration to inform the geometry and materiality needed to achieve specific motions through pneumatic actuation. As shown in the final print set, locomotion, gripping, and human interaction can be accomplished in soft robots using only one pneumatic input by fully utilising the geometric freedom and variable materiality of Polyjet printing.

The printable materials on the Stratasys $\mathrm{J} 750$ proved to be more prone to tearing and bursting than anticipated, and this limited the possible combinations of segments of actuation in complex soft robots. Although limitations were found in what is possible through one pneumatic input and with the soft printed materials, the actuators in this research demonstrate that complex motion can be achieved through one pneumatic input. The extension and bending sections gave similar deformation results to what had been expected, however, the twisting actuato significantly outperformed what had been anticipated, and the resulting bend angle was much larger than any other that was found in the literature.

Across soft robotic research, a wide range of applications have been identified that take advantage of the inherent compliance of soft actuators. This portfolio demonstrates a method for design and fabrication of soft robots that, with parametric modifications, can be applied to a number of these scenarios.
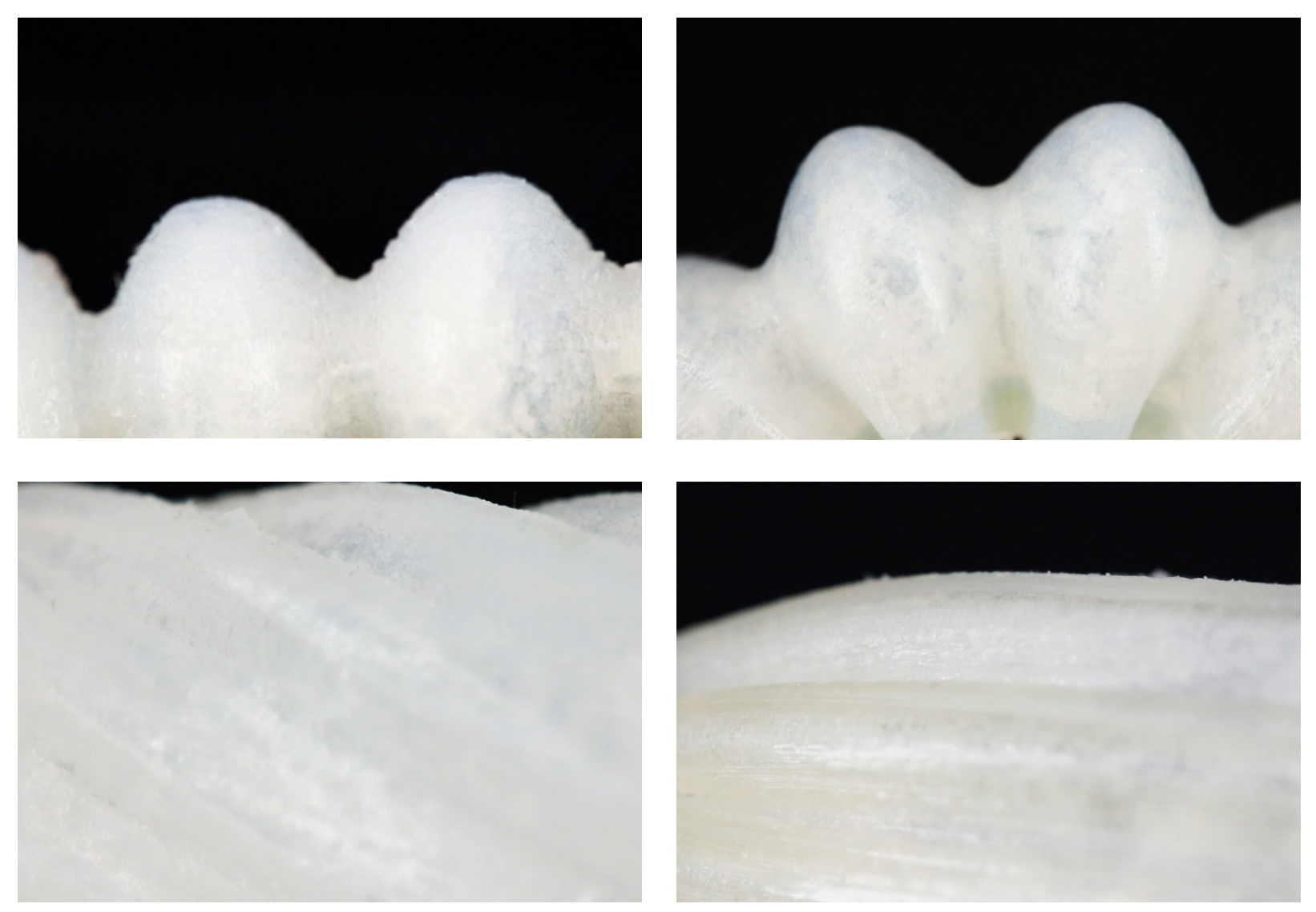

Figure 100. Close-up of the primary motions in their inflated states. 


\section{Figure list}

\section{All figures not cited have been produced by the author.}

Figure 4. Adapted from Heepe, L., \& Gorb, S. N.

(2014). Different adehsion mechanisms

resisting the separation of two surfaces.

Annual Review of Materials Research, 44(1),

173-203. [Diagram]. Retrieved from https://

www.annualreviews.org/doi/10.1146/

annurev-matsci-062910-100458

\section{References}

Arzt, E., Gorb, S., \& Spolenak, R. (2003). From micro to nano contacts in biological attachment devices. Proceedings of the National Academy of Sciences, 100(19), 10603-10606. https://doi. org/10.1073/pnas.1534701100

Autumn, K. (2006, March 1). How gecko toes stick: The powerful fantastic adhesive used by geckos is made of nanoscale hairs that engage tiny forces, inspiring envy among human imitators. American Scientist. https://link.galegroup.com/ apps/doc/A143278796/AONE?sid=Ims

Autumn, K., Sitti, M., Liang, Y. A., Peattie, A. M., Hansen, W. R., Sponberg, S., Kenny, T. W., Fearing, R., Israelachvili, J. N., \& Full, R. J. (2002). Evidence for van der Waals adhesion in gecko setae. Proceedings of the National Academy of Sciences, 99(19), 12252-12256. https://doi. org/10.1073/pnas.192252799

Barclift, M. W., \& Williams, C. B. (2012). Examining variability in the mechanical properties of parts manufactured via polyjet direct 3D printing. 6-8. Bartlett, N. W., Tolley, M. T., Overvelde, J. T. B., Weaver, J. C., Mosadegh, B., Bertoldi, K., Whitesides, G. M., \& Wood, R. J. (2015). A 3D-printed, functionally graded soft robot powered by combustion. Science, 349(6244), 161-165. https://doi. org/10.1126/science.aab012

Benyus, J. M. (1997). Biomimicry: Innovation inspired by ature (1st ed..). Morrow.
Bhushan, B. (2009). Biomimetics: Lessons from naturean overview. Philosophical Transactions of the Royal Society A: Mathematical, Physical and Engineering Sciences, 367(1893), 1445-1486. https://doi.org/10.1098/rsta.2009.0011 Cianchetti, M., Laschi, C., Menciassi, A., \& Dario, P. (2018). Biomedical applications of soft robotics. Nature Reviews Materials, 3(6), 143-153. https://doi.org/10.1038/s41578-018-0022-y

Cianchetti, M., Ranzani, T., Gerboni, G., De Falco, I., Laschi, C., \& Menciassi, A. (2013). STIFF-FLOP surgical manipulator: Mechanical design and experimental characterization of the single module. 2013 IEEE/RSJ International Conference on Intelligent Robots and Systems, 3576-3581. https://doi.org/10.1109/IROS.2013.6696866

Clode, D. (2017). The Third Thumb Project [Portfolio]. Dani Clode Design. https://www. daniclodedesign.com/thethirdthumb

Connolly, F., Polygerinos, P., Walsh, C. J., \& Bertoldi,

K. (2015). Mechanical Programming of Soft Actuators by Varying Fiber Angle. Soft Robotics, 2(1), 26-32. https://doi.org/10.1089/ soro.2015.0001

Connolly, F., Walsh, C. J., \& Bertoldi, K. (2017). Automatic design of fiber-reinforced soft actuators for trajectory matching. Proceedings of the National Academy of Sciences, 114(1), 51-56. https://doi org/10.1073/pnas.1615140114 
Darekar, B., Naik, P., Unde, J., \& Ohol, S. S. (2019). Development of Multi-chamber Pneumatic Twist Actuator for Soft robot [Portfolio]. https:// bhushandarekar.com/project/development-ofmulti-chamber-pneumatic-twist-actuator-forsoft-robot/

de Mestral, G. (1955). Velvet type fabric and method of producing same (United States Patent No. US2717437A). https://patents.google.com/ patent/US2717437A/en

Drotman, D., Jadhav, S., Karimi, M., deZonia, P., \& Tolley M. T. (2017). 3D printed soft actuators for a legged robot capable of navigating unstructured terrain. 2017 IEEE International Conference on Robotics and Automation (ICRA), 5532-5538. https://doi.org/10.1109/ICRA.2017.7989652

Ecoflex ${ }^{\text {TM }}$ 00-30 Product Information. (n.d.). Smooth-On Inc. Retrieved 13 January 2020, from https:// www.smooth-on.com/products/ecoflex-00-30/

Federle, W., Riehle, M., Curtis, A. S. G., \& Full, R. J. (2002). An Integrative Study of Insect Adhesion: Mechanics and Wet Adhesion of Pretarsal Pads in Ants. Integrative and Comparative Biology, 42(6), 1100-1106. https://doi.org/10.1093/ icb/42.6.1100

Festo. (2012). Bionic handling assistant: Safe humanmachine interaction [Company]. Bionic Learning Network. https://www.festo.com/group/en/ $\mathrm{cms} / 10241 . \mathrm{htm}$

Festo. (2019). Bionic soft arm: Modular pneumatic lightweight robot [Company]. Bionic Learning Network. https://www.festo.com/group/en/ $\mathrm{cms} / 13527 . \mathrm{htm}$

Frankel, L., \& Racine, M. (2010). The Complex Field of Research: For Design, through Design, and about Design. 12

Frayling, C. (1994). Research in Art and Design. Royal College of Art Research Papers, 1(1). http:// researchonline.rca.ac.uk/384/

Galloway, K. C., Becker, K. P., Phillips, B., Kirby, J., Licht, S., Tchernov, D., Wood, R. J., \& Gruber, D. F. (2016) Soft Robotic Grippers for Biological Sampling on Deep Reefs. Soft Robotics, 3(1), 23-33. https:// doi.org/10.1089/soro.2015.0019

Ge, L., Dong, L., Wang, D., Ge, Q., \& Gu, G. (2018). A digital light processing $3 \mathrm{D}$ printer for fast and high-precision fabrication of soft pneumatic actuators. Sensors and Actuators A: Physical, 273, 285-292. https://doi.org/10.1016/j. sna.2018.02.041

Glick, P., Suresh, S. A., Ruffatto, D., Cutkosky, M., Tolley, M. T., \& Parness, A. (2018). A Soft Robotic Gripper With Gecko-Inspired Adhesive. IEEE Robotics and Automation Letters, 3(2), 903-910. https://doi.org/10.1109/LRA.2018.2792688

Gu, Z., Li, S., Zhang, F., \& Wang, S. (2016). Understanding Surface Adhesion in Nature: A Peeling Model. Advanced Science, 3(7), 1500327. https://doi. org/10.1002/advs.201500327

Gul, J. Z., Sajid, M., Rehman, M. M., Siddiqui, G. U., Shah, I., Kim, K.-H., Lee, J.-W., \& Choi, K. H. (2018). 3D printing for soft robotics - a review. Science and Technology of Advanced Materials, 19(1), 243-262. https://doi.org/10.1080/14686996.20
18.1431862

Hamidreza, M., \& Hu, D. L. (2012). Friction enhancement in concertina locomotion of snakes. Journal of The Royal Society Interface, 9(76), 3067-3080 https://doi.org/10.1098/rsif.2012.0132

Hanington, B. M., \& Martin, B. (2012). Universal methods of design: 100 ways to research complex problems, develop innovative ideas, and design effective solutions (Digital ed..). Rockport Publishers. https://ebookcentral.proquest.com/ lib/vuw/detail.action?docID=3399583

Hanna, G., Jon, W., \& Barnes, W. P. J. (1991). Adhesion and Detachment of the Toe Pads of Tree Frogs. Journal of Experimental Biology, 155(1), 103125.

Heepe, L., \& Gorb, S. N. (2014). Biologically Inspired Mushroom-Shaped Adhesive Microstructures. Annual Review of Materials Research, 44(1) 173-203. https://doi.org/10.1146/annurevmatsci-062910-100458

llievski, F., Mazzeo, A. D., Shepherd, R. F., Chen, X., \& Whitesides, G. M. (2011). Soft Robotics for Chemists. Angewandte Chemie International Edition, 50(8), 1890-1895. https://doi org/10.1002/anie.201006464

Kier, W. M., \& Smith, A. M. (2002). The Structure and Adhesive Mechanism of Octopus Suckers. Integrative and Comparative Biology, 42(6), 1146-1153. https://doi.org/10.1093/ $\mathrm{icb} / 42.6 .1146$

Kier, W. M., \& Smith, K. K. (1985). Tongues, tentacles and trunks: The biomechanics of movement in muscular-hydrostats. Zoological Journal of the Linnean Society, 83(4), 307-324. https://doi. org/10.1111/j.1096-3642.1985.tb01178.x

Krüger, J., Lien, T. K., \& Verl, A. (2009). Cooperation

of human and machines in assembly lines. CIRP Annals, 58(2), 628-646. https://doi. org/10.1016/j.cirp.2009.09.009

Li, X., Tao, D., Lu, H., Bai, P., Liu, Z., Ma, L., Meng, Y., \& Tian, Y. (2019). Recent developments in geckoinspired dry adhesive surfaces from fabrication to application. Surface Topography: Metrology and Properties, 7(2), 023001. https://doi. org/10.1088/2051-672X/ab1447

Marchese, A. D., Onal, C. D., \& Rus, D. (2014). Autonomous Soft Robotic Fish Capable of Escape Maneuvers Using Fluidic Elastomer Actuators. Soft Robotics, 1(1), 75-87. https:// doi.org/10.1089/soro.2013.0009

Martinez, R. V., Branch, J. L., Fish, C. R., Jin, L., Shepherd, R. F., Nunes, R. M. D., Suo, Z., \& Whitesides, G. M. (2013). Robotic Tentacles with ThreeDimensional Mobility Based on Flexible Elastomers. Advanced Materials, 25(2), 205212. https://doi.org/10.1002/adma.201203002 MGrip. (n.d.). [Company]. Soft Robotics. Retrieved 18 December 2019, from https://www. softroboticsinc.com/mgrip

Milton, A., \& Rodgers, P. (2013). Research methods for product design. Laurence King Publishing Ltd.

Ngo, T. D., Kashani, A., Imbalzano, G., Nguyen, K. T. Q., \& Hui, D. (2018). Additive manufacturing (3D printing): A review of materials, methods, 
applications and challenges. Composites Part B: Engineering, 143, 172-196. https://doi org/10.1016/j.compositesb.2018.02.012

Patel, D. K., Sakhaei, A. H., Layani, M., Zhang, B., Ge, Q., \& Magdassi, S. (2017). Highly Stretchable and UV Curable Elastomers for Digital Light Processing Based 3D Printing. Advanced Materials, 29(15), 1606000. https://doi. org/10.1002/adma.201606000

Peele, B. N., Wallin, T. J., Zhao, H., \& Shepherd, R.

F. (2015). 3D printing antagonistic systems of artificial muscle using projection stereolithography. Bioinspiration \& Biomimetics, 10(5), 055003. https://doi.org/10.1088/17483190/10/5/055003

Peressadko, A., \& Gorb, S. N. (2004). When Less Is More: Experimental Evidence for Tenacity Enhancement by Division of Contact Area. The Journal of Adhesion, 80(4), 247-261. https:// doi.org/10.1080/00218460490430199

Polygerinos, P., Wang, Z., Galloway, K. C., Wood, R.

J., \& Walsh, C. J. (2015). Soft robotic glove for combined assistance and at-home rehabilitation. Robotics and Autonomous Systems, 73, 135-143. https://doi. org/10.1016/j.robot.2014.08.014

Ranzani, T., Gerboni, G., Cianchetti, M., \& Menciassi,

A. (2015). A bioinspired soft manipulator for minimally invasive surgery. Bioinspiration \& Biomimetics, 10(3), 035008. https://doi. org/10.1088/1748-3190/10/3/035008

Rizzo, N. W., Gardner, K. H., Walls D.J, Keiper-Hrynko,
N. M., Ganzke, T. S., \& Hallahan, D. L. (2006). Characterization of the structure and composition of gecko adhesive setae. Journa of The Royal Society Interface, 3(8), 441-451. https://doi.org/10.1098/rsif.2005.0097

Rus, D., \& Tolley, M. T. (2015). Design, fabrication and control of soft robots. Nature, 521(7553), 467475. https://doi.org/10.1038/nature14543 Schaffner, M., Faber, J. A., Pianegonda, L., Rühs,

P. A., Coulter, F., \& Studart, A. R. (2018) $3 \mathrm{D}$ printing of robotic soft actuators with programmable bioinspired architectures. Nature Communications, 9(1), 1-9. https://doi. org/10.1038/s41467-018-03216-w

Shepherd, R. F., Ilievski, F., Choi, W., Morin, S. A. Stokes, A. A., Mazzeo, A. D., Chen, X., Wang, M., \& Whitesides, G. M. (2011). Multigait soft robot. Proceedings of the National Academy of Sciences, 108(51), 20400-20403. https://doi. org/10.1073/pnas.1116564108

Shih, B., Christianson, C., Gillespie, K., Lee, S., Mayeda, J., Huo, Z., \& Tolley, M. T. (2019). Design Considerations for 3D Printed, Soft, Multimaterial Resistive Sensors for Soft Robotics. Frontiers in Robotics and Al, 6. https:// doi.org/10.3389/frobt.2019.00030

SLA vs. DLP: Compare Resin 3D Printers (2020 Guide). (n.d.). Formlabs. Retrieved 13 January 2020, from https://formlabs.com/blog/resin-3dprinter-comparison-sla-vs-dlp/

Tolley, M. T., Shepherd, R. F., Mosadegh, B., Galloway, K. C., Wehner, M., Karpelson, M., Wood, R.
J., \& Whitesides, G. M. (2014). A Resilient, Untethered Soft Robot. Soft Robotics, 1(3), 213223. https://doi.org/10.1089/soro.2014.0008

Trivedi, D., Rahn, C. D., Kier, W. M., \& Walker, I. D. (2008). Soft robotics: Biological inspiration, state of the art, and future research. Applied Bionics and Biomechanics, 5(3), 99-117. https://do org/10.1080/11762320802557865

Vincent, J. F. V., Bogatyreva, O. A., Bogatyrev, N. R., Bowyer, A., \& Pahl, A.-K. (2006). Biomimetics: Its practice and theory. Journal of The Royal Society Interface, 3(9), 471-482. https://doi. org/10.1098/rsif.2006.0127

Vogel, M. J., Steen, P. H., \& Probstein, R. F. (2010). Capillarity-based switchable adhesion Proceedings of the National Academy of Sciences, 107(8), 3377-3381. https://doi. org/10.1073/pnas.0914720107

Whitesides, G. M. (2016, May 26). Soft Robotics [Lecture]. H.C. Ørsted Lecture, Denmark. https://youtu.be/6j7FB4pCumU

Yap, H. K., Jeong Hoon Lim, Nasrallah, F., Goh, J. C. H., \& Yeow, R. C. H. (2015). A soft exoskeleton for hand assistive and rehabilitation application using pneumatic actuators with variable stiffness. 2015 IEEE International Conference on Robotics and Automation (ICRA), 4967-4972. https://doi.org/10.1109/ICRA.2015.7139889 Yap, Hong Kai, Ng, H. Y., \& Yeow, C.-H. (2016). HighForce Soft Printable Pneumatics for Soft Robotic Applications. Soft Robotics, 3(3), 144-158. https://doi.org/10.1089/soro.2016.0030
Yirmibesoglu, O. D., Morrow, J., Walker, S., Gosrich, W., Cañizares, R., Kim, H., Daalkhaijav, U., Fleming, C., Branyan, C., \& Menguc, Y. (2018). Direct 3D printing of silicone elastomer soft robots and their performance comparison with molded counterparts. 2018 IEEE International Conference on Soft Robotics (RoboSoft), 295-302. https://doi.org/10.1109/ ROBOSOFT.2018.8404935

Zhang, Y.F., Zhang, N., Hingorani, H., Ding, N., Wang, D., Yuan, C., Zhang, B., Gu, G., \& Ge, Q. (2019). Fast-Response, Stiffness-Tunable Soft Actuator by Hybrid Multimaterial 3D Printing. Advanced Functional Materials, 29(15), 1806698. https:// doi.org/10.1002/adfm.201806698

Zhu, M., Mori, Y., Wakayama, T., Wada, A., \& Kawamura S. (2019). A Fully Multi-Material ThreeDimensional Printed Soft Gripper with Variable Stiffness for Robust Grasping. Soft Robotics, 6(4), 507-519. https://doi.org/10.1089/ soro.2018.0112 
Well, Actuate(ly)...: Parametric Multi-Material 3D Printed Soft Robotics Patrick Coulson

2020 